C-A/AP/\#171

September 2004

\title{
Results from the RHIC PC CNI Polarimeter for 2003
}

\author{
O. Jinnouchi, I.G. Alekseev, A. Bravar, G. Bunce, S. Dhawan, \\ H. Huang, V. Hughes, G. Igo, V. Kanavets, K. Kurita, Z. Li, \\ W. Lozowski, W.W. MacKay, H. Okada, S. Rescia, T. Roser, \\ N. Saito, H. Spinka, D.N. Svirida, D. Underwood, C. Whitten and J. Wood
}

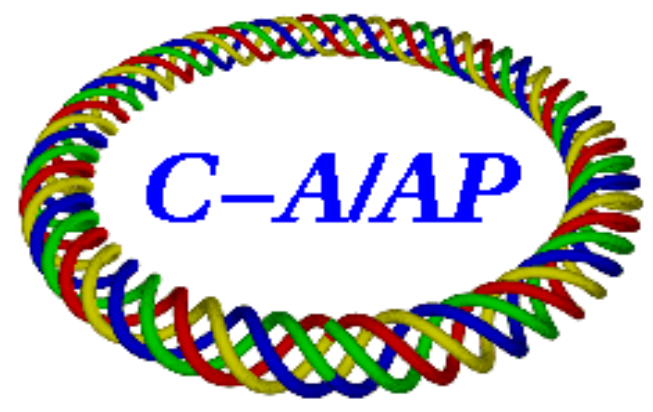

Collider-Accelerator Department Brookhaven National Laboratory Upton, NY 11973 


\title{
RESULTS FROM THE RHIC PC CNI POLARIMETER FOR 2003
}

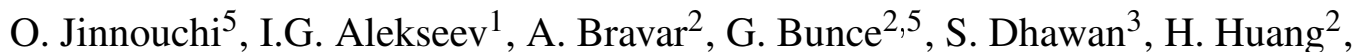 \\ V. Hughes ${ }^{3}$, G. Igo ${ }^{4}$, V. Kanavets ${ }^{1}$, K. Kurita ${ }^{6}$, Z. Li $^{2}$, W. Lozowski ${ }^{7}$, W.W. MacKay ${ }^{2}$, \\ H. Okada ${ }^{8}$, S. Rescia ${ }^{2}$, T. Roser ${ }^{2}$, N. Saito ${ }^{8}$, H. Spinka ${ }^{9}$, \\ D.N. Svirida ${ }^{1}$, D. Underwood ${ }^{9}$, C. Whitten ${ }^{4}$ and J. Wood ${ }^{4}$ \\ (1) Institute for Theoretical and Experimental Physics, B. Cheremushkinskaya 25, Moscow, \\ 117259, Russia \\ (2) Brookhaven National Laboratory, Upton, NY 11973, USA \\ (3) Yale University, New Haven, CT 06511, USA \\ (4) UCLA, Los Angeles, CA 90095, USA \\ (5) RIKEN BNL Research Center, Upton, NY 11973, USA \\ (6) Rikkyo University, Toshima-ku, Tokyo 171-8501, Japan \\ (7) Indiana University Cyclotron Facility, Bloomington, IN 47405, USA \\ (8) Kyoto University, Kyoto 606-8502, Japan \\ (9) Argonne National Laboratory, Argonne, IL 60439, USA
}

\section{INTRODUCTION}

The RHIC proton-carbon CNI polarimeters use pC elastic scattering in the coulombnuclear interference region to measure the beam polarization. Sensitivity to polarization is due to the coulomb spin-flip amplitude that is also responsible for the proton anomalous magnetic moment. The interference term arising from this amplitude, electromagnetic spinflip $\times$ hadronic spin nonflip, is calculable, but an additional interference term, from a hadronic spin flip amplitude $\times$ electromagnetic spin nonflip, is not.

The polarimeter analyzing power $A_{N}$ was determined at $22 \mathrm{GeV}$ by measuring the beam polarization in an external beam at the AGS, experiment E925 [1], while simultaneously measuring the CNI asymmetry in the AGS ring, experiment E950 [2]. E925 used proton-proton elastic scattering in a larger $t$ region $\left(-t=0.15(\mathrm{GeV} / \mathrm{c})^{2}\right)$, where the analyzing power was known (and non-zero), from polarized target experiments. $A_{N}$ for pC CNI was determined to $\pm 30 \%$ at $22 \mathrm{GeV}$ (Fig. 1)[2]. The analyzing power for RHIC at $100 \mathrm{GeV}$ will be determined for the first time using a new polarized atomic hydrogen gas jet target in RHIC, over the next two years (2004-5).

The RHIC polarimeters include a carbon target that can be introduced into the RHIC beam for the measurements, and silicon detectors that measure the energy and time 


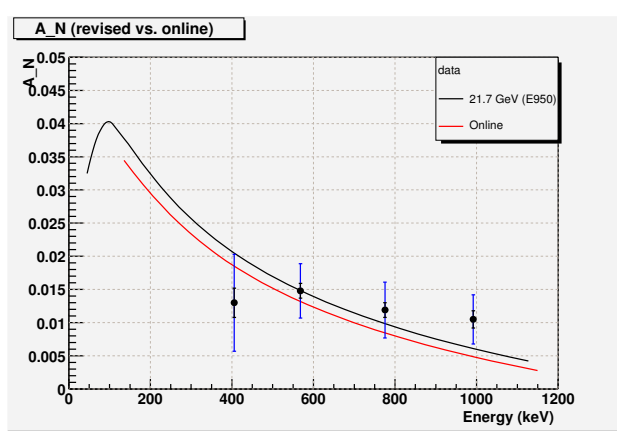

FIGURE 1. Analyzing power for proton-carbon elastic scattering for $22 \mathrm{GeV}$ protons, vs. recoil carbon energy. Dots with error bars are the data points in our energy domain from E950 [2]. Top curve is a fit to the E950 data points, including points not shown at higher recoil energy, from Larry Trueman. [3] Lower curve is the fit used for the 2003 online analysis.

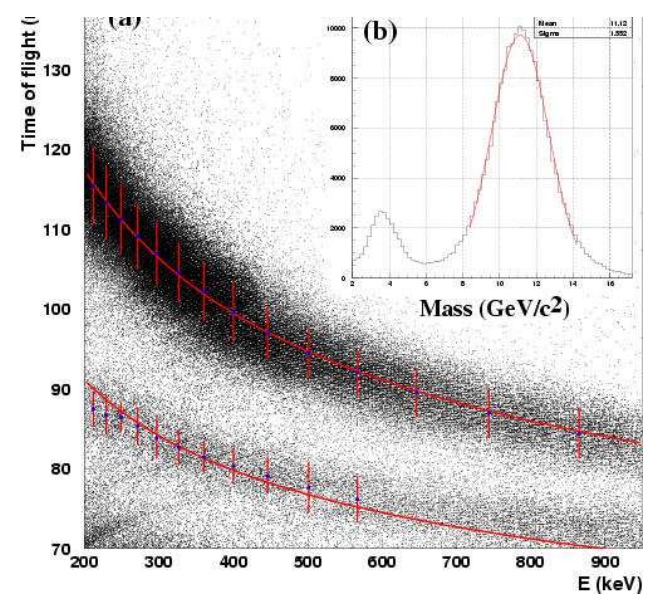

FIGURE 2. (a) The time of flight is plotted as a function of kinetic energy of the detected particle. (b) Sub-figures show the reconstructed invariant mass distribution, discussed in the text. The carbon mass peak $\left(11.18 \mathrm{GeV} / \mathrm{c}^{2}\right)$ is clearly separated from an alpha mass peak $\left(3.7 \mathrm{GeV} / \mathrm{c}^{2}\right)$.

of arrival of the recoil carbon ions. For very small angle scattering, elastic reactions dominate, and measurement of the recoil gives predominantly elastic events. The CNI region that we measure covers $-t=0.006$ to $0.03(\mathrm{GeV} / \mathrm{c})^{2}$, or carbon energies of 300 $\mathrm{keV}$ to $1.3 \mathrm{MeV}$ range. The carbon recoil polar angle is nearly 90 degrees. The time of arrival provides time-of-flight for the recoil, by comparing to the time the rf-bunched beam crosses the target. The flight times are of order $50 \mathrm{~ns}$ to $100 \mathrm{~ns}$ for detectors at $15 \mathrm{~cm}$ from the target. This is ideal, since this is a quiet time-most backgrounds arrive close to the crossing time. The time-of-flight and energy measurements are used to identify carbon, see Fig. 2. An asymmetry is measured for counts in a left detector vs. a right detector, $\varepsilon_{L R}=\left(N_{L}-N_{R}\right) /\left(N_{L}+N_{R}\right)$, after selection of carbon events. The polarization is obtained from $P=-\varepsilon_{L R} / A_{N}$. The beam polarization can also be obtained from the asymmetry in counts observed for beam polarization up vs. polarization down, for each detector. In practise, both the left-right asymmetry and the polarization up-down asymmetry are used to measure and control systematic errors in the measurement. 


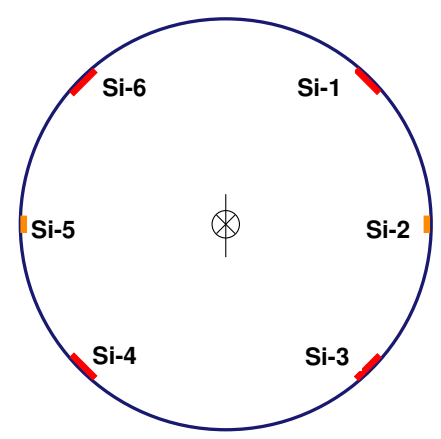

FIGURE 3. The layout of the silicon detectors inside the $15 \mathrm{~cm}$ radius vacuum pipe of each RHIC polarimeter. The polarized proton beam direction is into the paper, and the carbon target is represented by the vertical line at the center of the vacuum pipe.

In the following sections we describe the RHIC polarimeters and the measurements during the 2003 RHIC run.

\section{EXPERIMENTAL SETUP}

The RHIC polarimeters are located near the 12 o'clock intersection region, with separate polarimeters near Q4 in each beam. The beams are referred to as the Blue and Yellow beams. A schematic of the polarimeters is shown in Fig. 3. The RHIC polarized proton beam passes through an ultra-thin carbon ribbon target, and carbon recoils from CNI scattering are observed in six silicon strip detectors placed as shown. Very thin carbon ribbon targets have been developed at IUCF [4]. A typical target is $2.5 \mathrm{~cm}$ long, 3.5$\mu \mathrm{g} / \mathrm{cm}^{2}$ thick (150 $\AA$ ) and 5- $\mu \mathrm{m}$ wide. The target is mounted on a mechanism which rotates into the beam, with a choice of 3 vertical and 3 horizontal targets. The detector has $10 \times 24 \mathrm{~mm}^{2}$ total active area, divided into 12 strips of $10 \times 2 \mathrm{~mm}^{2}$ each. The thickness of the detectors are $400 \mu \mathrm{m}$, fully depleted with the operation bias voltage of $100 \mathrm{~V}$. The strips are made by $p^{+}$-doping (B implantation) to a depth of $150 \mathrm{~nm}$ on the n-type Si bulk on the side facing the target. The back side is the $n^{+}$-doped layer with an Al contact.

The six detectors are mounted inside of the vacuum chamber with readout preamplifier boards directly attached to the chamber detector ports through vacuum feedthrough connectors.

Figure 2 shows a scatter plot of time of flight versus energy for one silicon strip in the polarimeter. The silicon detectors are $15 \mathrm{~cm}$ from the target, and the RHIC bunch length was about $2 n s$. The insets in the figure show mass distributions derived from velocity and energy. The carbon and $\alpha$ peaks are clear, with little background under the carbon peak. The beam polarization is measured by counting the number of events in the carbon band in each strip versus the azimuthal angle of the strip around the beam (Fig. 3). A vertical polarization generates a left-right asymmetry in the detectors and a radial polarization generates an up-down asymmetry in the detectors. The rates are very high, so we chose a readout system without dead time based on waveform digitizers (WFD) [5]. The WFDs consist of a high frequency video ADC chip (used for laptop 
screens) and a Xilinx FPGA.

The waveform from each strip was digitized every $2.36 \mathrm{~ns}$, and pulse height and time of flight, compared to the RHIC rf clock, was determined in real time. The $1.18 \mathrm{~ns}$ timing resolution is obtained through the interpolation algorithm, and compared to a look-up table which accepted the carbon band (as in Fig. 2). On-board scalers kept the number of events for each strip, and for each beam bunch. The 55 beam bunches of polarized protons in RHIC for the 2003 run, spaced $212 n s$ apart, alternated in polarization sign. Therefore, the on-board scalers collected data for both signs, and for bunches set up with zero polarization, for each strip. 48 strips were read out, 8 for each detector (Fig. 3), and the same WFDs were used for blue and yellow measurements. Also, the orientation of the strips for the left and right 90 degree detectors (Fig. 3) were set up with the strips perpendicular to the beam direction, to measure the polar scattering angle. The $45^{\circ}$ detectors were oriented along the beam direction to reduce the azimuthal acceptance for each strip, reducing the rate compared to the $90^{\circ}$ central strips. Due to the multiple scattering in the target, the measurement of scattering angle gives only a weak constraint on elastic scattering. For the 2003 run, we typically had $4 \times 10^{12}$ protons in each ring, and $2 \times 10^{7}$ carbon elastic events were collected in about 20 seconds, with the target then rotated out of the beam. The data were then transfered to a PC, the asymmetry and various monitor asymmetries were calculated, and the result was sent automatically to the accelerator and experiments in minutes. A detailed description is given in [5].

\section{UPGRADES FOR 2003}

For the 2003 RHIC run we added the capability to store the carbon energy (pulse height and integral), time of arrival of the recoil (1/4 pulse height timing), and bunch number for each event for each silicon strip. Each WFD module was equipped with a $16 \mathrm{MB}$ SDRAM, which holds about $45 \mathrm{M}$ events in on board memory in a total of twelve modules. This readout mode (event mode) was in addition to the scaler mode where histograms are stored, that were previously used for asymmetries. The scaler mode contains the sum of events for each strip and each bunch number, for events passing a preselected banana cut of time of flight vs. recoil pulse height, corresponding to carbon events. A selected carbon energy range is required for the scaler results. Histograms are also kept by carbon energy bin for events within the banana cuts, for each strip, for,+, 0 polarization signs. The polarization signs are obtained from the bunch numbers, via downloaded CDEV information, and not through hardware signals.

RHIC operators selected the mode for collecting data, scaler or event+scaler. The event mode readout time from on board memory to a PC hard drive was about 5 minutes, so a typical pattern used was to use scaler mode for the measurement of 1 beam, with a quick result readout, followed by event mode for the second beam, alternating these between beams. Also, we typically took only one measurement at injection because the multiple scattering from the target increased the beam emittance. Polarization values reported during the run were based on scaler data, from either the scaler mode or from the event+scaler mode. This analysis uses the refined calibrations and event selection available from the event data. 

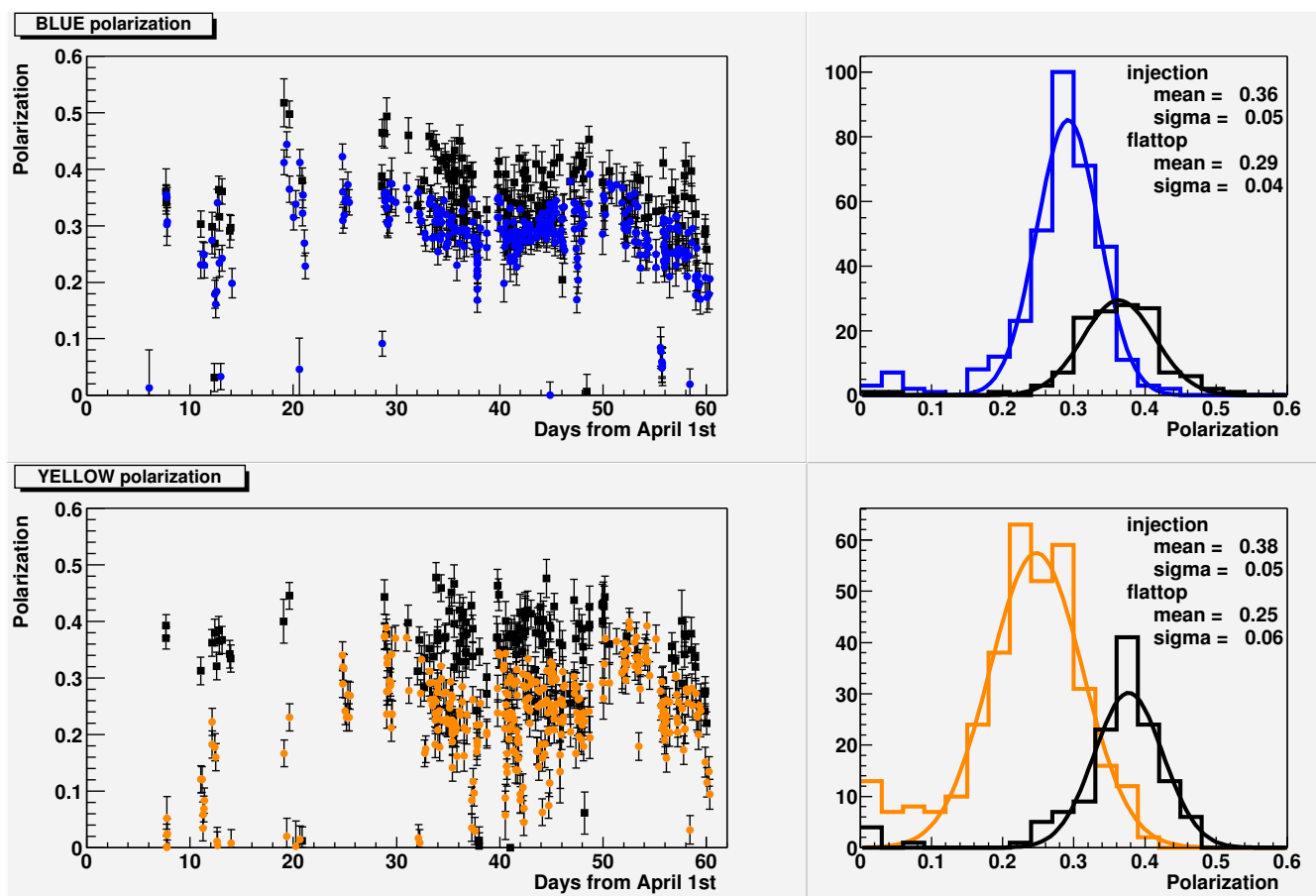

FIGURE 4. Asymmetry measurements made during the 2003 run, using the scaler mode readout. Top plots for BLUE and the bottom plots for YELLOW. In the left plots, each polarization measurement is shown as a data point, black points represent the injection measurements and colored ones are for the flattop measurements.

\section{POLARIMETER MEASUREMENTS DURING 2003 RUN}

Fig. 4 shows polarization measurements taken from the scalers during the run. These measurements combine the polarization sign $(+,-)$ data and the left-right (or up-down) data using a geometric mean (the square root formula). The data from during the run are shown for yellow beam injection $(24 \mathrm{GeV})$, and flattop $(100 \mathrm{GeV})$, and for blue beam. We also show a false asymmetry check as Fig. 5, where the 45 degree detectors are combined to cancel any real polarization effect: the cross asymmetry between, refering to Fig. 3, (\#1+\#4) vs. (\#3+\#6).

To summarize the observations from the run measurements: Vertical polarization is observed as expected. We also observed a radial polarization asymmetry for blue flattop throughout the run. The cross asymmetry (false asymmetry) for blue was non-zero. Finally, the t-dependence of the vertical polarization asymmetry in blue follows the curve for $A_{N}$ in Fig. 1, as it should for a real signal. However, the radial polarization asymmetry t-dependence for blue was flat, implying that this may be a false asymmetry. 

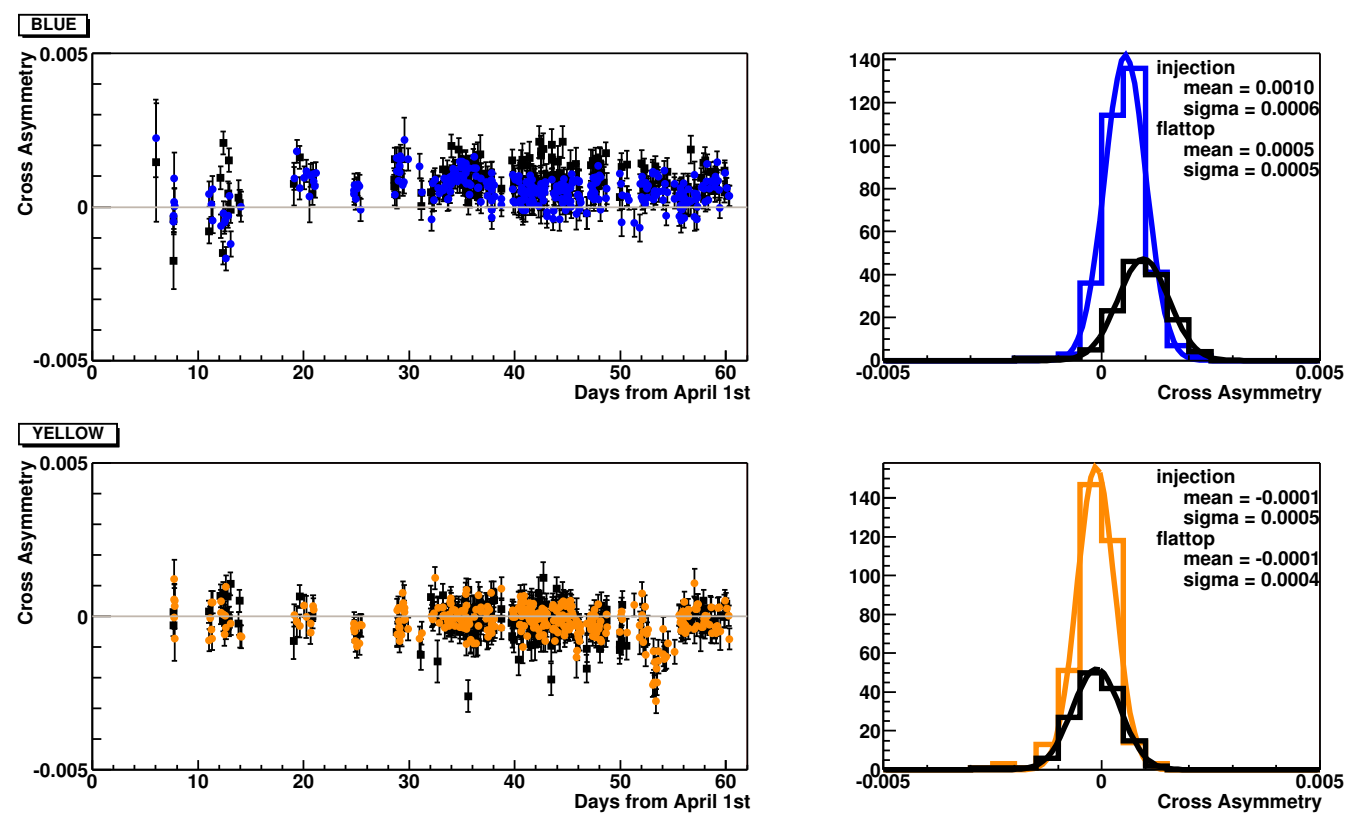

FIGURE 5. Unphysical asymmetry (cross asymmetry) from the scaler data. Top plots for BLUE and the bottom plots for YELLOW. In the left plots, each measurement is shown as a data point, black points represent the injection measurements and colored ones are for the flattop measurements.Deviation from zero indicates the fact that the wrong dead layer correction had been applied at the online level.

\section{OFFLINE, POSTRUN ANALYSIS PLAN}

The observed false cross asymmetry and the unexpected radial signal implied that different strips and/or detectors may have different behavior. For example, 45 degree detectors can generate a false up-down asymmetry from a real left-right asymmetry (pointed out by Vadim Kanavets) if there is a large difference in $A_{N}$ between the detectors. We therefore decided to treat each strip as an independent polarimeter, to compare the behavior for each strip.

To use each strip as a polarimeter, we measure the asymmetry for events from + polarization bunches vs. - polarization bunches, $\varepsilon_{+-}$, where we must normalize by the luminosity ratio for the $+/$ - polarization bunches, $\mathrm{R}$ :

$$
\varepsilon_{+-}=\left(N_{+}-R N_{-}\right) /\left(N_{+}+R N_{-}\right), \text {where } R=L_{+} / L_{-} .
$$

We used the counts in the 90 degree detectors (\#2+\#5) to determine $L_{+}$and $L_{-}$. Then

$$
P_{i}=\left(\varepsilon_{+-, i} / A_{N, i}\right)
$$

for strip $i$. $A_{N, i}$ is the analyzing power for the strip $i$, as determined from the fit to Fig. 1, weighted by the observed carbon energy distribution for strip $i$.

We used the event mode data to recalculate the silicon dead layer energy loss and the bunch crossing time. During the run this was done by approximating the energy loss vs. energy with a linear response with offset. This is a reasonable approximation only for small dead layers. Fig. 6 shows curves for energy loss vs. energy for carbon 

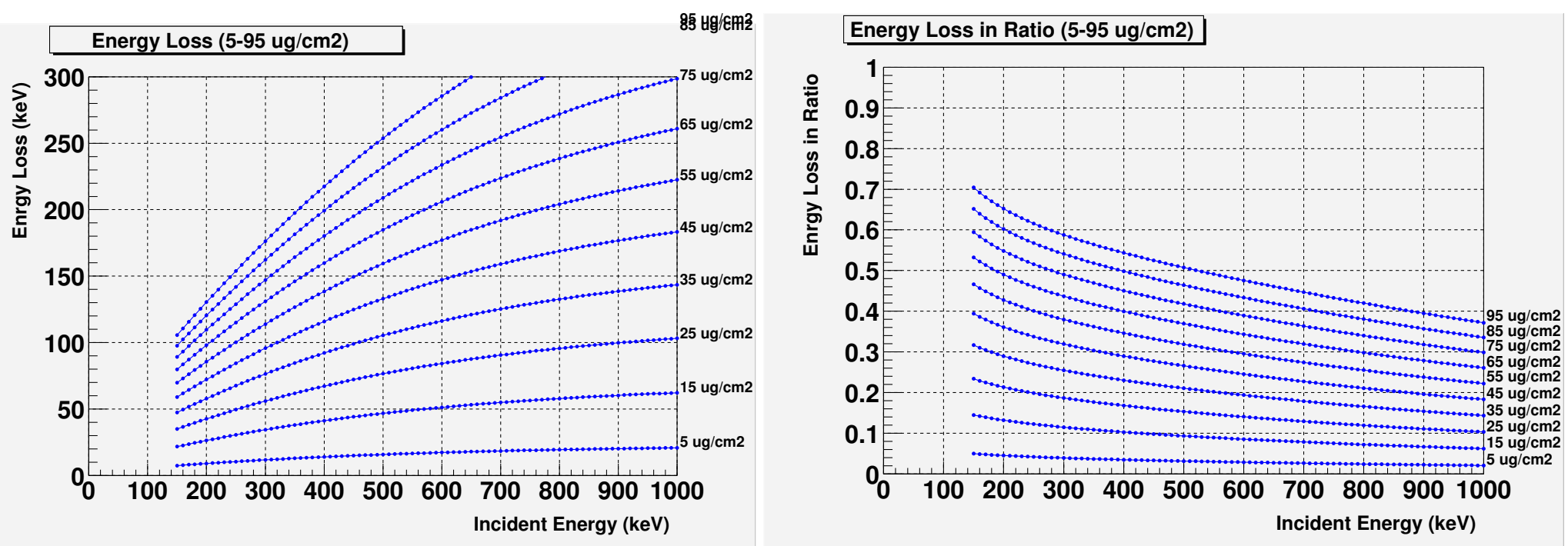

FIGURE 6. The left plot shows the energy loss, dE/dx, for different thickness dead layers, for carbon ions incident on silicon /citedEdx, vs. carbon energy. The right plot shows the fractional energy loss.

incident on silicon [6], and for the fractional energy loss vs. energy for different dead layer thicknesses. A linear relationship would give a flat line for fraction vs. energy, which is only reasonable for small thickness and larger incident energy domain, as seen in the figure. For the offline, the curves in the figure were described by a forth order polynomial in E, with the single parameter $w$, the deadlayer thickness. A fit was then made to the central value of the time-of-flight vs. energy banana (or, equivalently, to the carbon mass), with only two parameters: $w$ and the time offset $t_{0}$. This fit to the carbon mass was much improved from the linear approximation for the dead layer. Fig. 7 shows the dead layer thicknesses for each strip from this two parameter fit, done independently for each strip. As seen in the figure, the same detectors show nearly the same thickness for each strip. We then used the average thickness for each detector, indicated by the red points. None of the detectors came from the same wafer, so no correlation between detectors is expected. This new dead layer correction shifted the carbon energies from the online by about $+100 \mathrm{keV}$. This shift leads to a change in effective analyzing power from online of about $-10 \%$ (see Fig. 1.)

Fig. 1 also shows a lower curve marked "Online 2003". This curve was used for the effective analyzing power in the online results. A mistake was found in this curve, which is just a fit to the E950 data shown. The correct fit is shown as "Larry's fit to E950", the fit by Larry Trueman [3]. The revised fit increases $A_{N}$ by $10 \%$ from the online.

The two effects together, the new deadlayer and the fit correction, largely cancel in their effects on the effective analyzing power for the polarimeter in 2003.

For the event selection in the offline, we used a number of standard deviations from the central carbon mass. For the online, we used fixed time cuts from the carbon locus $(+/-12$ ns from the carbon locus, independent of energy). This is shown in Fig. 8. The offline cut was considerably tighter and more controled vs. background from, for example, alphas. For the offline, the carbon mass center and sigma was calculated for each run (some runs 

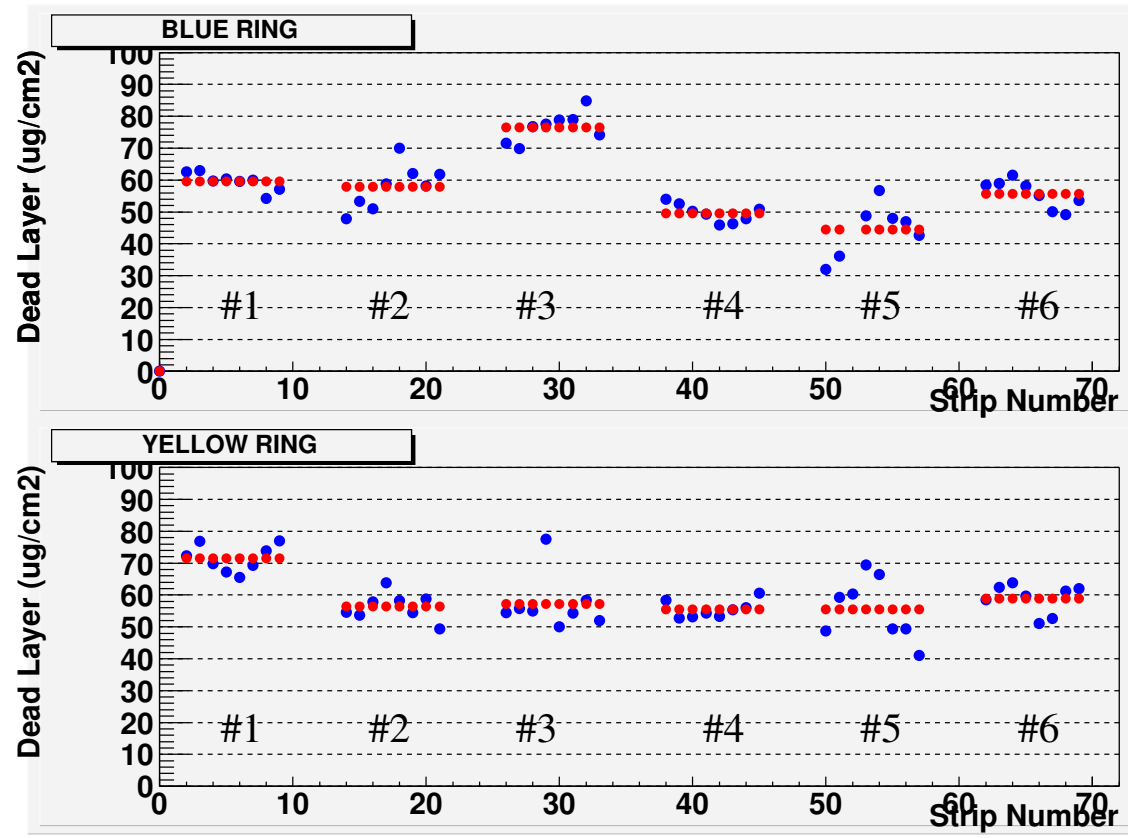

FIGURE 7. Measured deadlayer thickness for each strip, based on two parameter fit to carbon mass locus. A polynomial was used to describe $\mathrm{dE} / \mathrm{dx}$ vs. E for carbon incident on silicon.

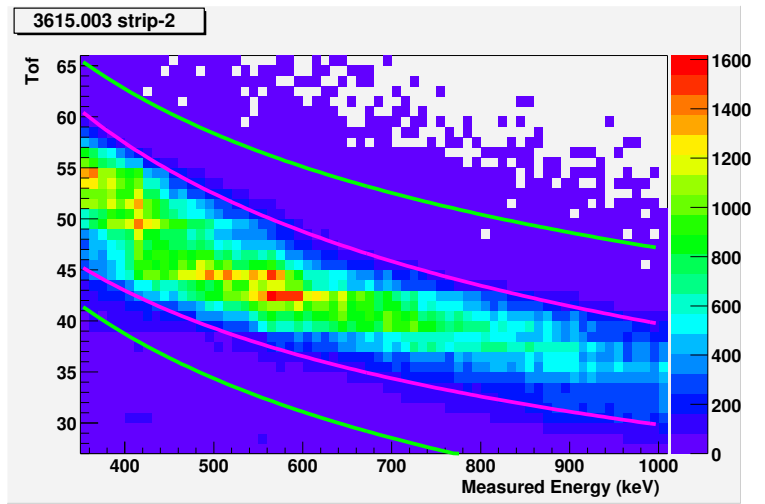

FIGURE 8. Comparing two cut criteria, two outside lines (light green) are the online cut condition, i.e. $+/-12$ nsec, and two lines inside are the cut for $3 \sigma$ deviation around the carbon mass.

had better timing resolution than others).

\section{OFFLINE RESULTS I}

We then calculated the polarization for each strip, for injection and flattop. Our intention was to compare the stability of the asymmetry for different carbon mass cuts, and to compare the 48 strips for each beam, for injection and flattop. To display the results, we convert the asymmetry to polarization using the effective analyzing power from 

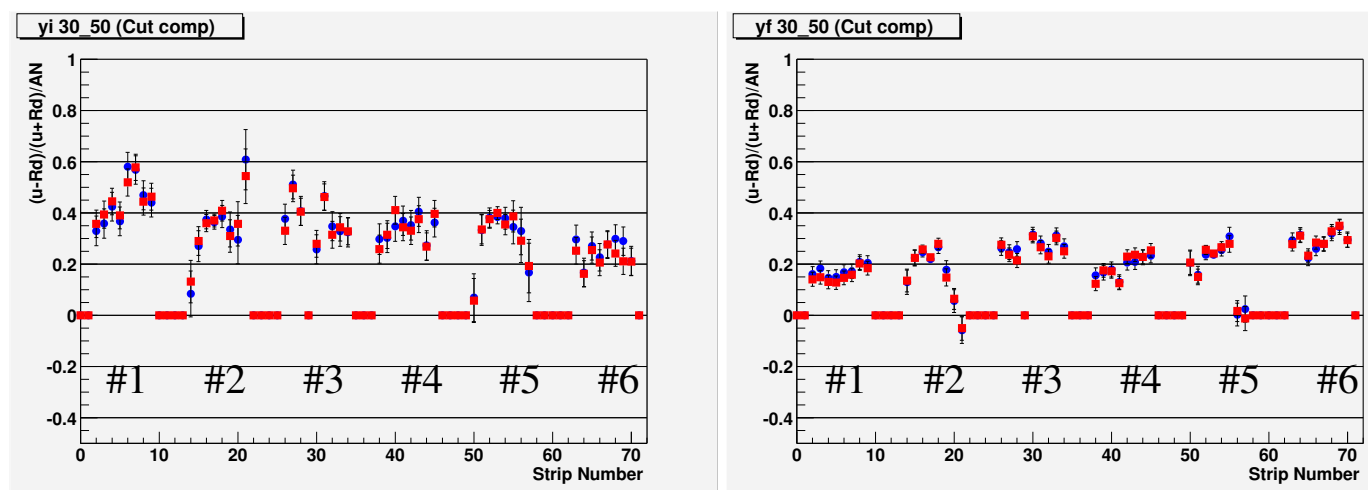

FIGURE 9. Beam polarization in yellow ring for 20 days accumulated, May 1-20, 2003. Each strip acts as an independent polarimeter. Both injection (left) and flattop (right) are shown, with statistical errors only. Two event selection cuts are shown, $1.5 \sigma$ (blue data points) and $3.0 \sigma$ (red data points) around the carbon mass. All bunches are assigned the same relative start time, $t_{0}$. The designation \#N refers to the silicon detector number in Fig. 3

weighting the fit shown in Fig. 1 by our energy distribution for each strip, and correcting for the azimuthal dependence of the analyzing power (the 45 degree detectors have a $\sqrt{2}$ lower analyzing power than the 90 degree detectors for vertical polarization). We also flip the sign for the detectors to beam-right. Roughly $A_{N}= \pm 0.012 \cos \phi$, with the + sign for detectors to beam left, and - sign for beam right detectors. For a vertical polarization only, all 48 detectors should give the same value for polarization.

Fig. 9 shows the result for yellow. Asymmetries for two cuts are shown: $1.5 \sigma$ and 3 $\sigma$ from the central carbon mass. We note several points. The results are fairly stable with the two cuts. The edge strips of the 90 degree detectors see many fewer carbon events and also show lower polarization (these detectors measure scattering polar angle, and most events are in 2 or 3 central strips). Strips within a 45 degree detector roughly agree, but the scatter seems non-statistical, particularly for injection (note that the error bars for injection are larger because we took fewer measurements there). The six detectors don't show the same polarization.

There is some structure between the detectors for the polarization results in yellow, which is noticable at flattop. Pairs of detectors, \#1 and \#4, \#2 and \#5, and \#3 and \#6 measure similar polarization. This is the structure that would be observed for a combination of vertical and radial polarization. Yellow flattop shows evidence of radial polarization. For yellow injection, a systematic error is evident for detector \#6 compared to the others.

Fig. 10 shows the blue result. In addition to the remarks made for yellow above, we also see that the polarization values are unstable with the mass cuts.

Due to the instability of the result for blue, we investigated the bunch dependence of the start time $t_{0}$. Fig. 11 shows the carbon mass peak position for each bunch, for 20 days of data, for blue flattop, for strip 27 (detector \#3). The mass shows a systematic variation with bunch number. A zoom of this strip also shows a mass shift for even vs. odd bunches. These shifts are presumably from $t_{0}$, the timing of the bunch crossing.

Fig. 12 shows the calculated carbon mass difference for + bunches vs. - bunches, for 

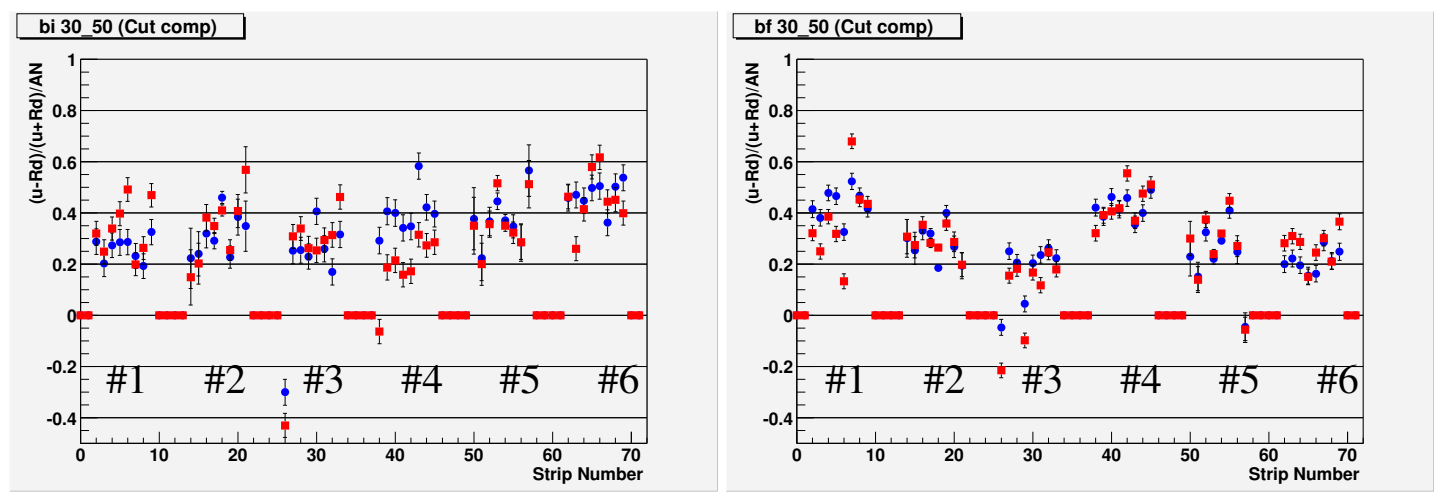

FIGURE 10. Beam polarization in blue ring for 20 days accumulated, May 1-20, 2003. Each strip acts as an independent polarimeter. Both injection and flattop are shown, with statistical errors only. Two event nass. All
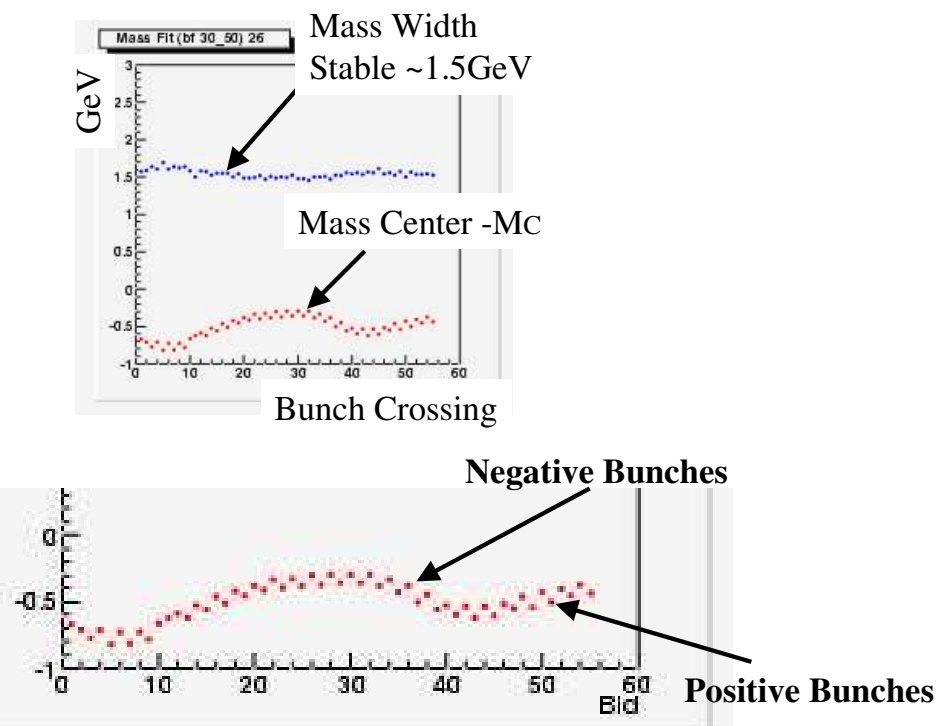

FIGURE 11. The top plot shows the calculated carbon mass for one strip, \#27 blue, for flattop measurements over 20 days, versus bunch number. $11 \mathrm{GeV}$ is subtracted from the carbon mass in these plots. A zoom is shown in the bottom figure.

blue and yellow at flattop. This is shown for each strip. We see that blue shows a very large fluctuation, vs. much smaller fluctuations for yellow.

We have not yet understood the origin of these variations, but we assume that the bunch rf time is at fault, and we have calculated $t_{0}$ for each bunch for each run and each strip. Our goal is to have a stable carbon mass to use to select events. 

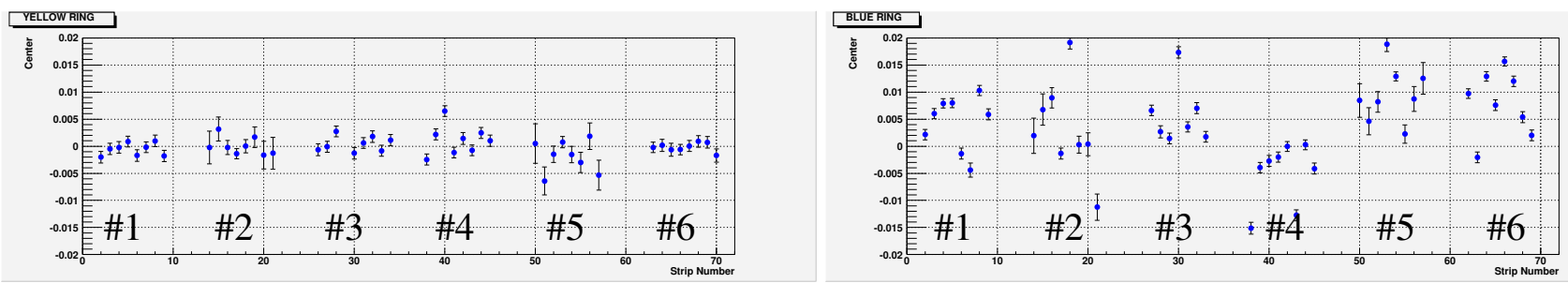

FIGURE 12. Carbon mass differences for + bunches vs. - bunches, for blue (right plot) and yellow (left plot) flattop, for accumulated data over 20 days.
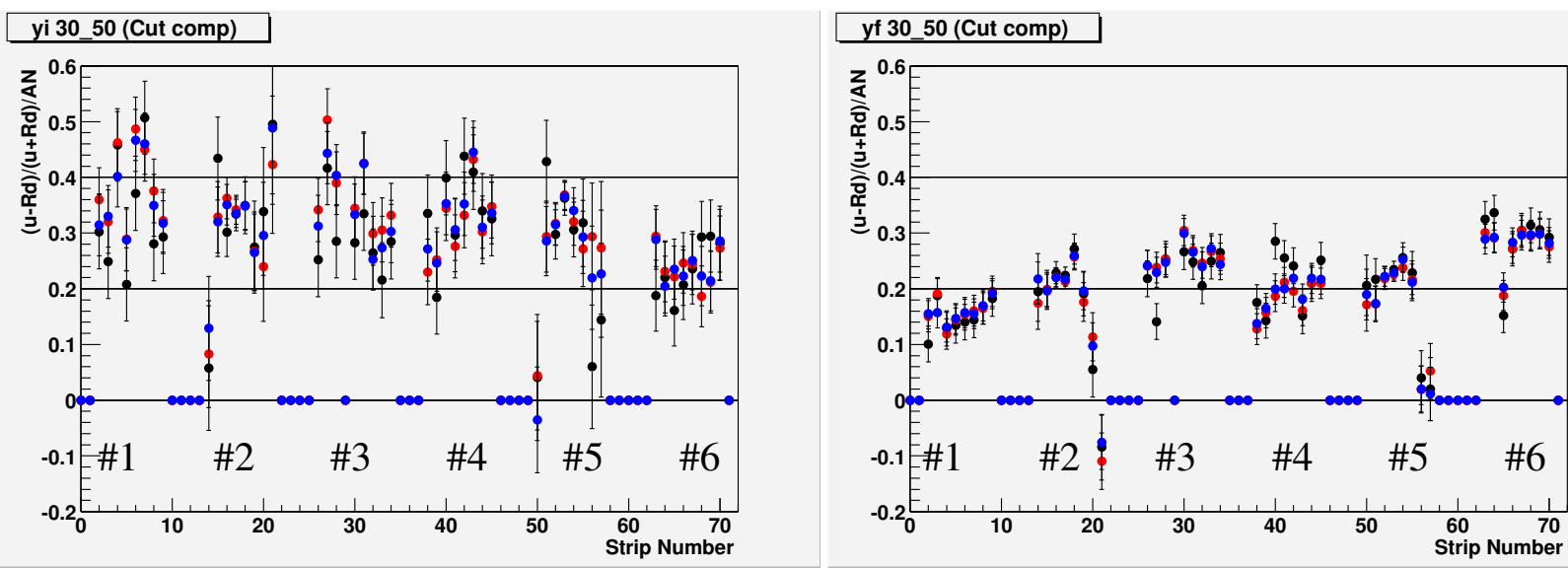

FIGURE 13. Beam polarization in yellow ring for 20 days accumulated, May 1-20, 2003. Each strip acts as an independent polarimeter. Both injection and flattop are shown, with statistical errors only. Three event selection cuts are shown, $1.0 \sigma$ (black), $2.0 \sigma$ (red), and $3.0 \sigma$ (blue) around the carbon mass. Bunches are assigned a relative start time, $t_{0}$, to center the carbon mass, for each strip and fill.

\section{OFFLINE RESULTS II}

Fig. 13 and Fig. 14 show the results for polarization for each strip after adjusting the rf bunch time $t_{0}$ to center the carbon mass for each bunch for each fill, and for each strip. The results are stable for different mass cuts.

With the stable results, we then discuss the differences for the strips. We have not understood the lower polarization measured for blue strip 27, and for the edge strips for the 90 degree detectors in blue and yellow. For strip 27, we had a very large variation in $t_{0}$ for + and - bunches. For the edge strips, the events there come from multiple scattering in the target [2]. We have decided to eliminate these strips from the measurement: strip 27 blue, and 2 edge strips from each edge of each 90 degree detector.

The blue injection polarization has general agreement for the remaining 39 strips. Blue flattop polarization has structure indicating a significant radial polarization.

Fig. 15 shows the difference in asymmetry measured with different mass cuts, normalized by an error that accounts for one set of data being a subset of the other [7]. This is shown for yellow flattop. The histogram is the projection. If we consider the excess beyond statistics to be a systematic error, we have $\sigma_{\text {sys }}=\sqrt{\sigma^{2}-1} \times \sigma_{\text {meas }}$. We then 

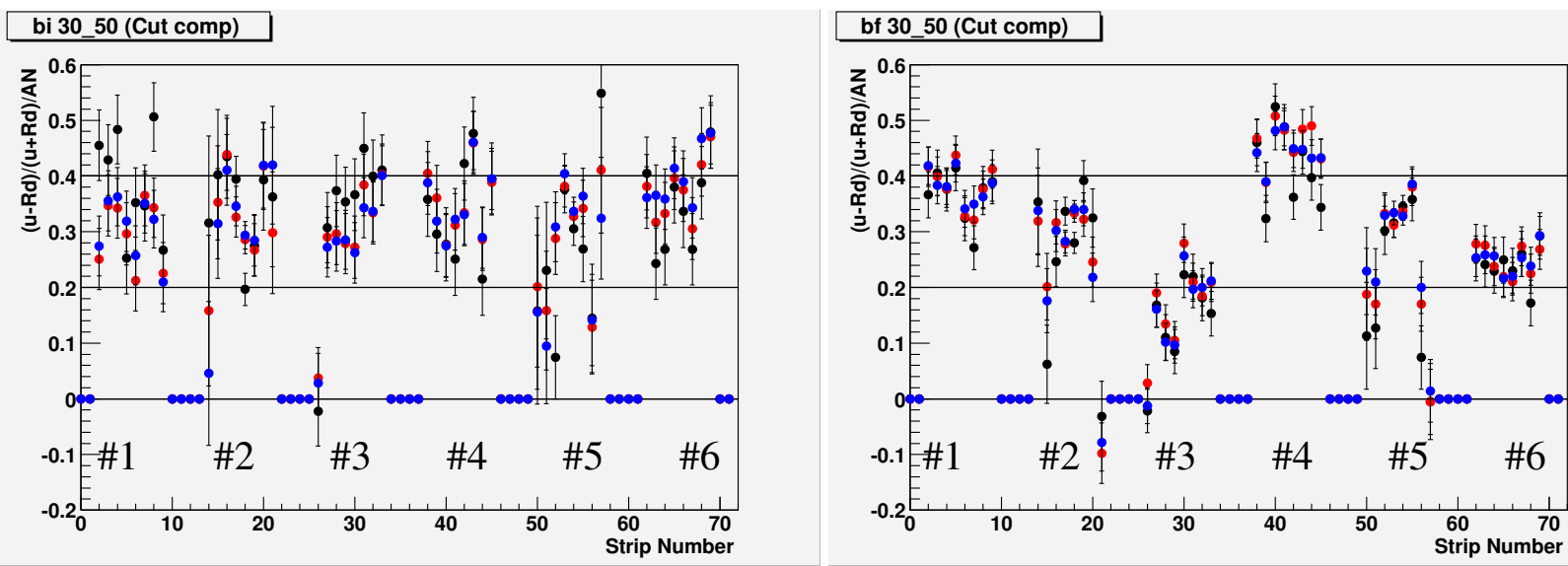

FIGURE 14. Beam polarization in blue ring for 20 days accumulated, May 1-20, 2003. Each strip acts as an independent polarimeter. Both injection and flattop are shown, with statistical errors only. Three event selection cuts are shown, $1.0 \sigma$ (black), $2.0 \sigma$ (red), and $3.0 \sigma$ (blue) around the carbon mass. Bunches are assigned a relative start time, $t_{0}$, to center the carbon mass, for each strip and fill.

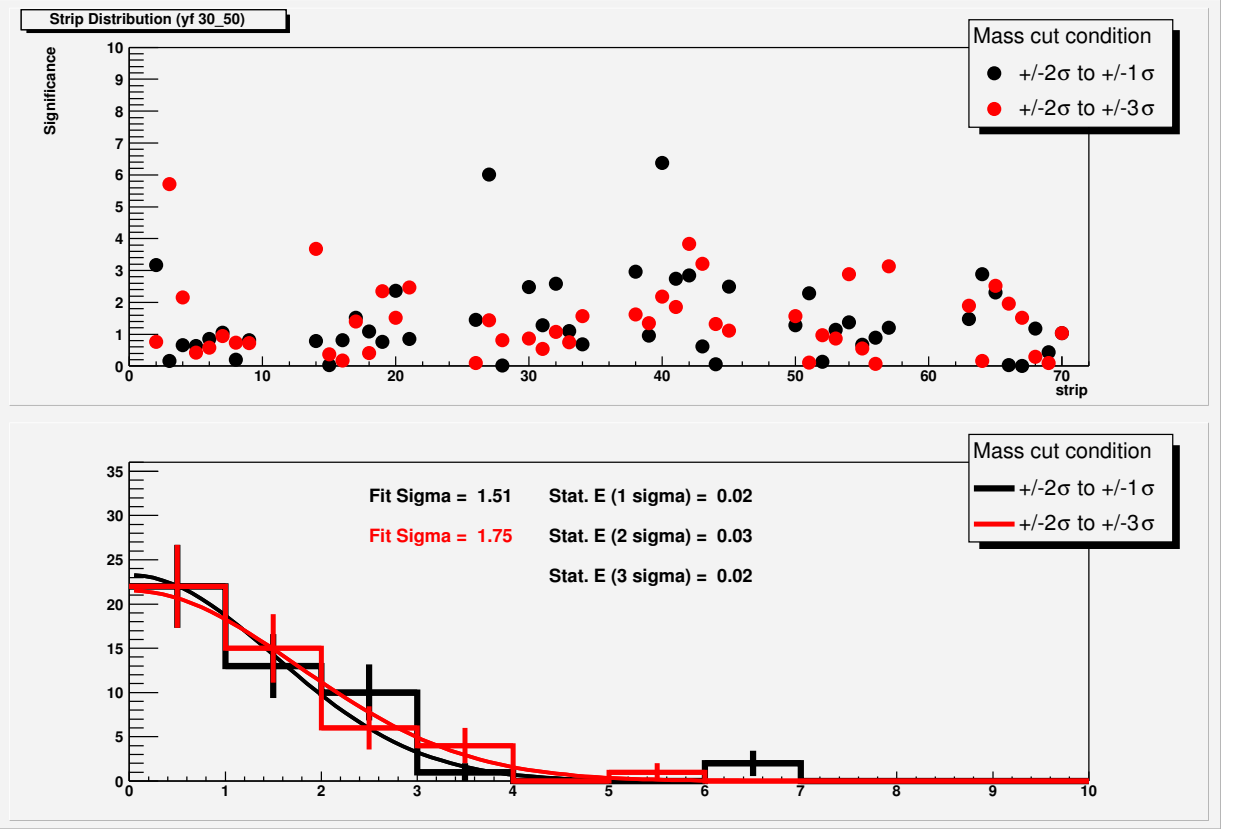

FIGURE 15. Study of asymmetry dependence on the recoil carbon mass cut. The top plot shows the difference in asymmetry measured for two mass cuts, $2 \sigma$ and $3 \sigma$ around the carbon mass, normalized by the statistical error. The statistical error takes into account that one set of data is a subset of the other. The histogram is a projection. The average significance should be 1 , if the errors are only statistical.

have $\sigma_{s y s}=0.5 \%$ for yellow flattop, $1.5 \%$ for yellow injection, $1.1 \%$ for blue flattop, and $1.3 \%$ for blue injection., where these numbers are for polarization. These systematic errors are fairly small.

We then studied the $t$-dependence of the measured asymmetries. A real beam polarization signal should have a $t$-dependence that follows the analyzing power, Fig. 1 . The 


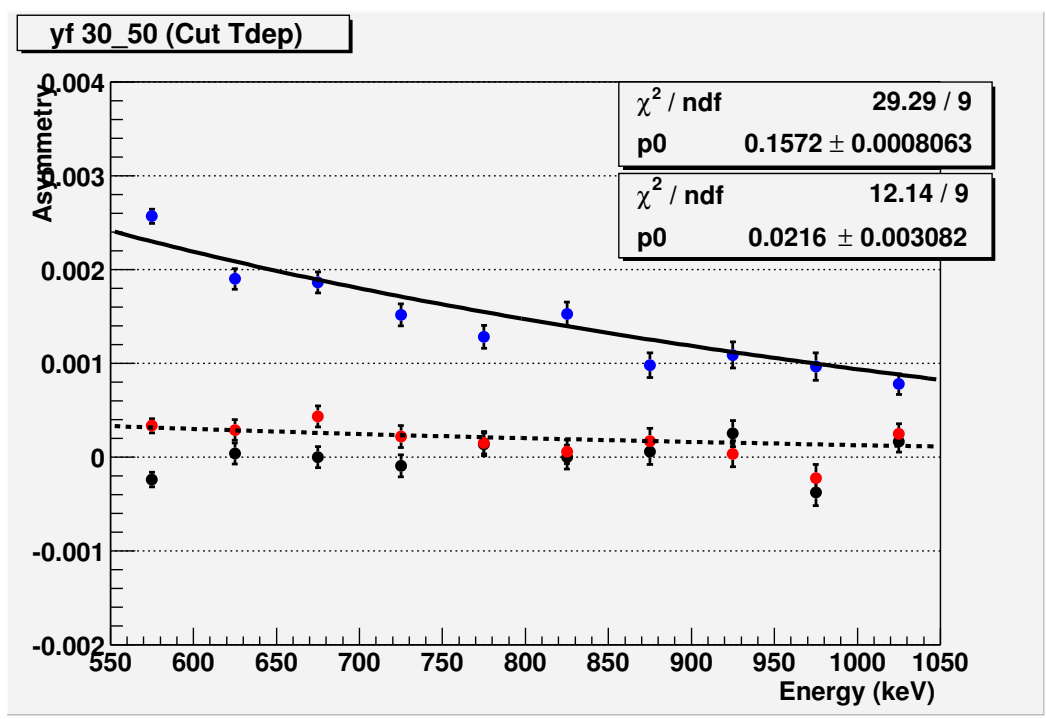

FIGURE 16. The four-momentum, $t$, dependence of the measured asymmetries for the yellow beam at flattop. The solid lines are fits to the left-right asymmetries (blue closed points) with $k \times A_{N}$. The dashed line is the fit for the flattop up-down asymmetries (red closed points) with $k^{\prime} \times A_{N}$. The black closed points around zero are the cross asymmetries.

results for the yellow beam are shown in Fig. 16. In this figure we see that the left-right asymmetry follows the expected dependence for flattop. The cross asymmetries are zero. A small up-down asymmetry at flattop has the expected $t$-dependence, consistent with interpreting the data from Fig. 13 as indicating some radial polarization.

Fig. 17 shows the $t$-dependence for the blue beam polarization measurements. Again, the left-right asymmetry follows $A_{N}(t)$, Fig. 1, for flattop. The cross asymmetries are zero. The up-down asymmetry at flattop follows $A_{N}(t)$, indicating a radial component of the polarization. We also show the ratio of up-down to right-left asymmetry vs. $-t$. The ratio is constant, with a $\chi^{2}=13$ for 8 degrees of freedom.

\section{ESTIMATES OF SYSTEMATIC ERRORS}

We have decided to estimate the systematic errors for two cases: for vertical polarization only (V), and for vertical and radial polarization both allowed $(\mathrm{V}+\mathrm{R})$. The systematic error is estimated from the error required to give a $\chi^{2} / n d f=1$. For the yellow flattop, the fits of the strip asymmetries to azimuthal angle $\phi$ are shown in Fig. 18. For vertical polarization only the asymmetry results for the 39 strips are divided by the analyzing power, including dependence on $\phi$. For the case where radial polarizaion is allowed, the figure shows the raw asymmetry divided by $A_{N}$. For vertical polarization only, the derived systematic error is $\pm 4 \%$ out of a yellow polarization of $22 \%$ for these runs. When radial polarization is allowed, a radial component is measured pointing at 12 degrees to the inside of the ring. The derived systematic error is then $\pm 1 \%$, out of $23 \%$ total polarization. 

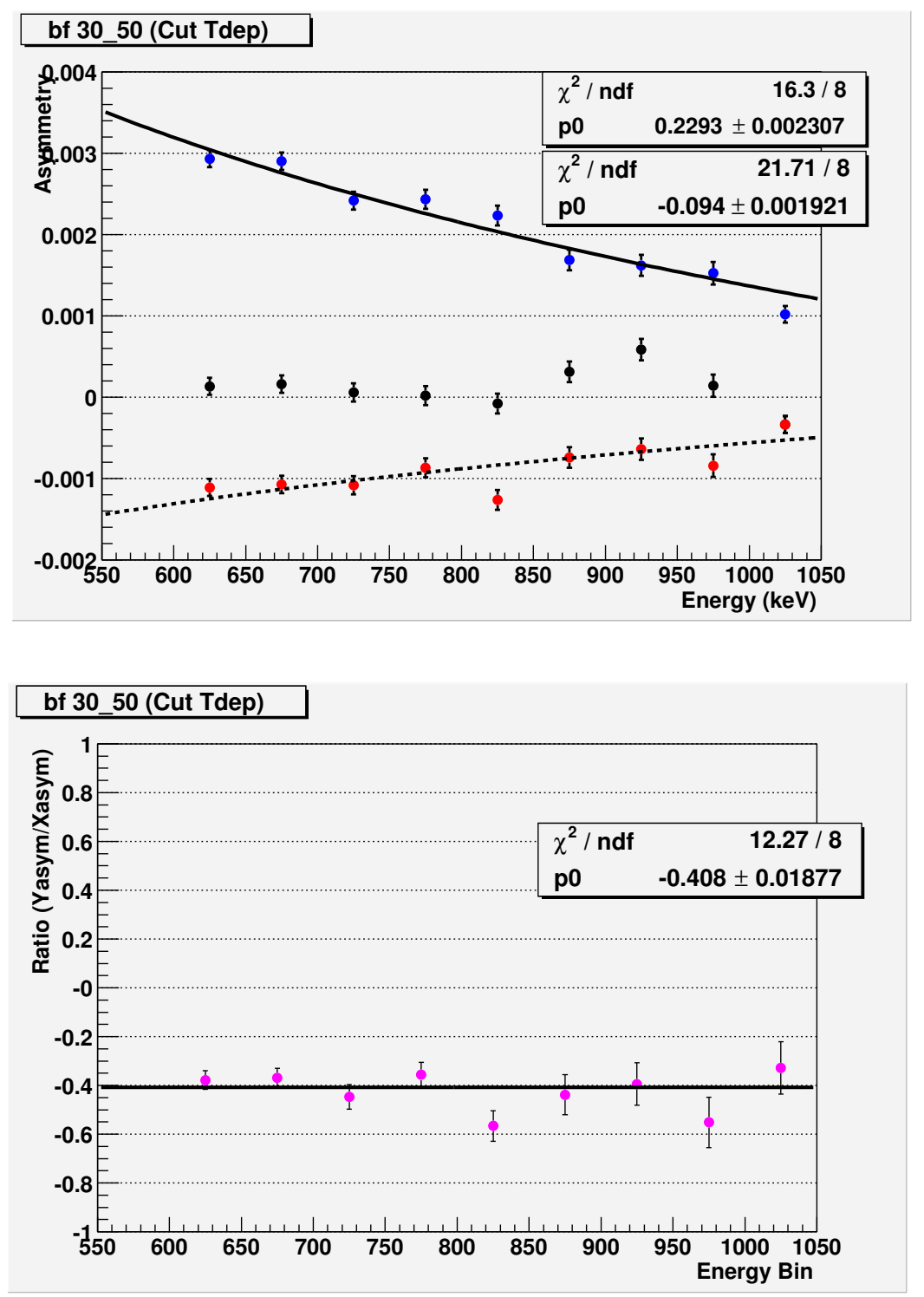

FIGURE 17. Same for the blue beam. The ratio of the up-down asymmetry to the left-right asymmetry, vs. $t$, is also shown.

Fig. 19 shows data and fits for the blue beam polarization measurements vs. detector azimuth. For vertical polarization only, the derived systematic error is $\pm 9 \%$, out of a polarization of $32 \%$ for these runs. When we allow a radial polarization, the radial component points to 17 degrees toward the inside of the ring, and the derived systematic error is $\pm 3 \%$ out of a polarization of $34 \%$. Table 1 shows the result for flattop.

Injection is shown in Fig. 20 for both yellow and blue. No significant radial polarization is observed for yellow or blue, and systematic errors are small. (Here the $\chi^{2}$ for blue without radial polarization is smaller than when radial polarization is allowed. This is our artifact of the treatment of the errors for the $45^{\circ}$ detectors for the top right figure.) 


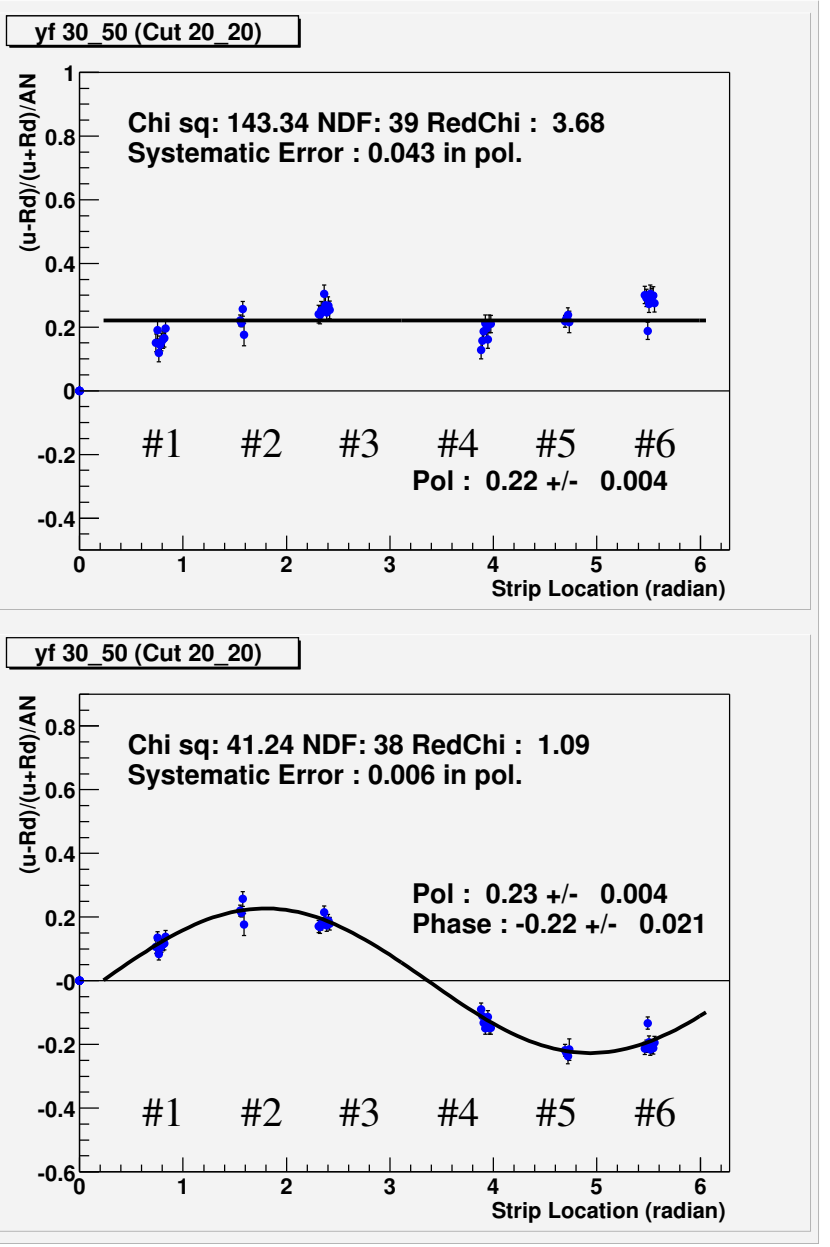

FIGURE 18. The top plot shows a fit of the measured polarizations for each of 39 strips for the yellow beam flattop, assuming a vertical polarization, vs. azimuthal angle of the detectors. The bottom plot shows the strip raw asymmetries divided by $A_{N}$, versus the detector phi. The top plot is fitted with a flat line, i.e. vertical polarization only. The bottom plot fit allows radial polarization.

TABLE 1. estimated systematic errors in different assumptions

\begin{tabular}{llrr} 
Condition & Ring & error & polarization \\
\hline Vertical polarization only & Blue & 0.09 & 0.32 \\
& Yellow & 0.04 & 0.22 \\
\hline Allow radial component & Blue & 0.03 & 0.34 \\
& Yellow & 0.01 & 0.23 \\
\hline
\end{tabular}

\section{DISCUSSION}

At the March 19, 2004 RHIC Spin Collaboration meeting, Joanna Kiryluk presented STAR local polarimeter data from the run. A small radial raw asymmetry is observed at flattop for the yellow beam $\varepsilon_{\text {radial }}^{\text {yellow }} / \varepsilon_{\text {vertical }}^{\text {yellow }}=-0.16 \pm 0.03$. None was observed for blue, 

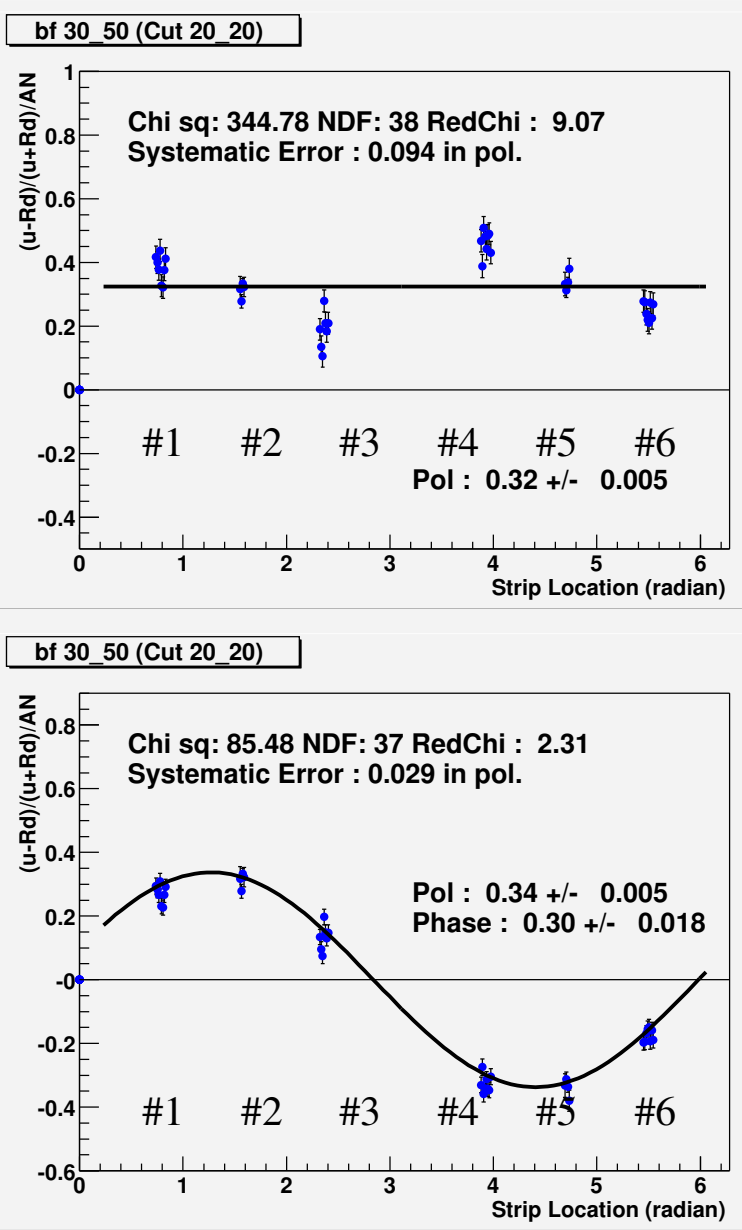

FIGURE 19. The top plot shows a fit of the measured polarizations for each of 39 strips for the blue beam flattop, assuming a vertical polarization, vs. azimuthal angle of the detectors. The bottom plot shows the strip raw asymmetries divided by $A_{N}$, versus the detector phi. The top plot is fitted with a flat line, i.e. vertical polarization only. The bottom plot fit allows radial polarization.

$\varepsilon_{\text {radial }}^{\text {blue }} / \varepsilon_{\text {vertical }}^{\text {blue }}=-0.03 \pm 0.02$. This was for running with the STAR spin rotators off, and PHENIX spin rotators on. At this meeting also, Naohito Saito showed PHENIX local polarimeter measurements for data with all spin rotators off. The radial raw asymmetry for yellow was $\varepsilon_{\text {radial }}^{\text {yellow }} / \varepsilon_{\text {vertical }}^{\text {yellow }}=0.19 \pm 0.21$. For blue, $\varepsilon_{\text {radial }}^{\text {blue }} / \varepsilon_{\text {vertical }}^{\text {blue }}=0.15 \pm 0.02$.

A radial polarization in yellow at flattop was expected, due to the loss of one of the yellow snake magnets. With one yellow snake operating as a partial snake, a horizontal component of the polarization was predicted. The amount of radial polarization depends on the RHIC beam energy ( $G \times \gamma$, with $G$ the anomalous magnetic moment coefficient of proton, and $\gamma$ the Lorentz factor) and position of the polarimeter in the ring. This was apparently observed by the pC CNI polarimeter, STAR. PHENIX did not have sufficient sensitivity (PHENIX only ran for a short time with the spin rotators off and the yellow measurement were done with a fill with very small polarization, $\mathrm{P}=8 \%$ ). Unfortunately, the beam energy isn't known with sufficient precision to predict the degree of radial 

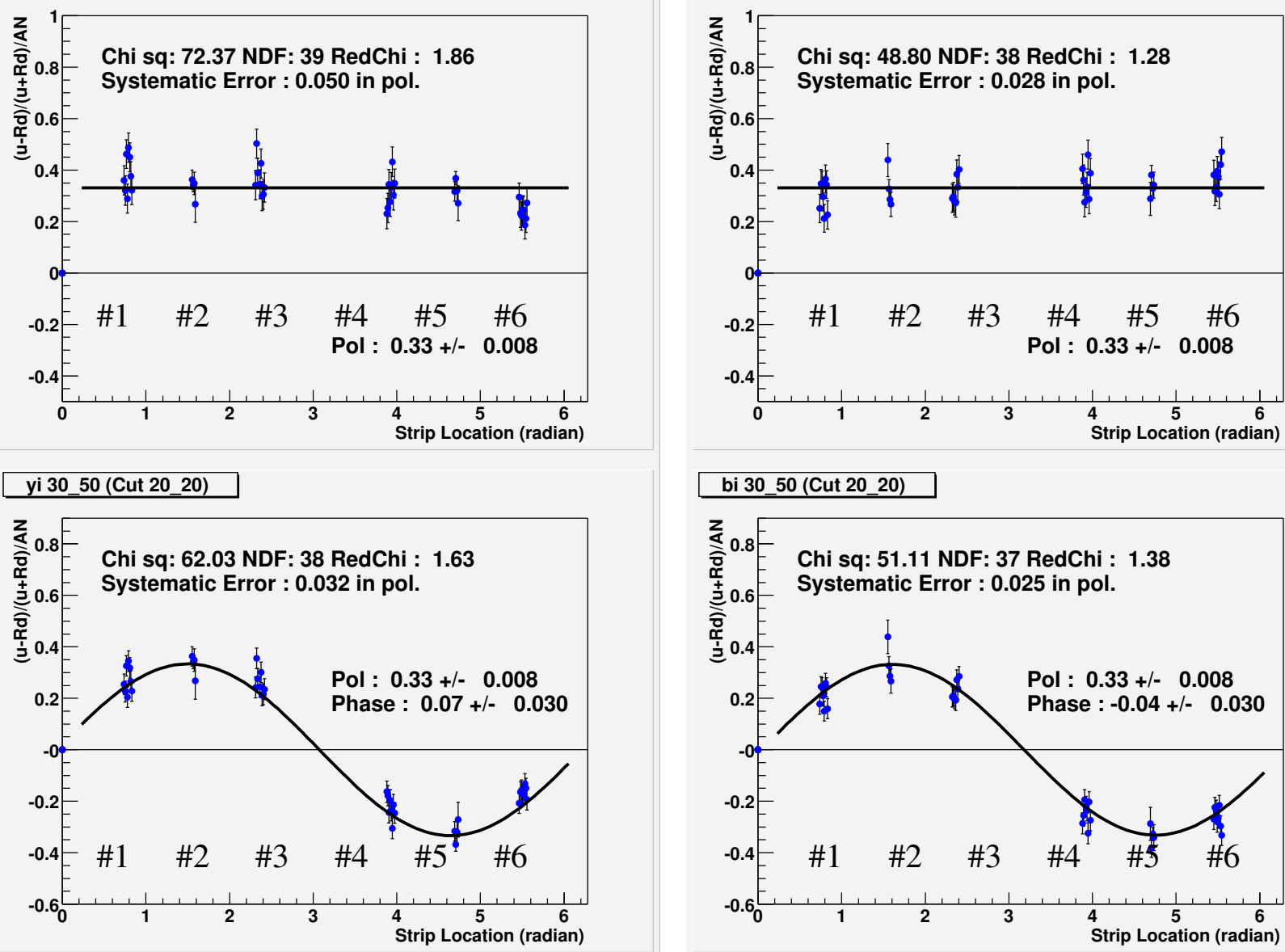

FIGURE 20. The top plots show a fit of the measured polarizations for each of 39 strips for the yellow (left) and blue (right) beam injection, assuming a vertical polarization, vs. azimuthal angle of the detectors. The bottom plots show the strip raw asymmetries divided by $A_{N}$, versus the detector phi. The top plot fits use a flat line, i.e. vertical polarization only. The bottom plot fits allow radial polarization.

polarization at each location. However, it appears reasonable to attribute the observed yellow radial polarization at flattop to a real effect. Therefore, we assign the yellow beam systematic error from the spread of results for the 39 strips to be $\pm 1 \%$ in polarization.

No radial polarization in blue was expected.

Waldo Mackay discussed possible radial polarization in blue from a mistuning of the snakes and spin rotators. He does not expect a radial polarization of the observed size in blue. None is observed by STAR, and a statistically significant radial asymmetry is observed by PHENIX.

The blue radial asymmetry is either real, or it is a mistake, which we have not yet uncovered. The measured blue right-left asymmetry at injection matches the AGS polarimeter measurements, so that the measurement of the vertical polarization for the blue polarimeter appears to be robust. The $t$-dependence of the blue radial signal (updown asymmetry) matches the $t$-dependence of the analyzing power, implying that the 
signalis real. No false cross asymmetry is observed. The run-dependence of the radial signal is stable. Thus, all measurements from the CNI polarimeter indicate a real radial polarization. Therefore, we conclude that we should use the systematic error for blue from the case where a radial polarization is allowed. This is $\pm 3 \%$ in polarization at flattop.

\section{SUMMARY}

Differences from the online included a corrected curve for $A_{N}(t)$, evaluation of the dead layers for the detectors using a parameterization of $\mathrm{dE} / \mathrm{dx}$ for carbon incident on silicon, and calculation of the bunch timing for each bunch to center the data at the recoil carbon mass. After this work, the mass was stable vs. energy, the event selection dependence was small, the cross asymmetries (false asymmetries) were zero, and the $t$-dependence of the asymmetries matched the analyzing power.

The measured systematic error for the polarization measurements in 2003 were $\pm 3 \%$ in polarization, for which we have taken the largest measured systematic error. This error comes from an evaluation of the blue and yellow measurements at injection and flattop, treating each silicon strip (39 strips for each polarimeter) as independent polarimeters. We have allowed a radial polarization, as well as vertical. We have observed a small radial polarization in yellow and a large radial polarization in blue, both at flattop, and both with good consistency and with the expected t-dependence of a real signal. A radial polarization was expected in yellow, and none was expected in blue. The systematic errors in yellow were less than blue, $\pm 1 \%$ in polarization.

The polarization in blue at flattop increased by $3 \%$, from $28.5 \%$ to $31.4 \%$, from the online result. The yellow flattop polarization increased by $1 \%$, from $24.2 \%$ to $25.2 \%$. This is for all runs from April 15 to May 30, excluding the special pp2pp runs. A spreadsheet has been distributed to the experiments, for STAR and PHENIX, in the Appendix, giving the new polarization results for each polarimeter run. The data include bunch selection for each experiment, where non-colliding bunches are excluded.

\section{A. POLARIZATION RESULTS FOR EACH POLARIMETER RUN}

The tables in the following three appendices give the RHIC polarimeter results for run 3. The first two appendices give the polarization values based on event mode data, analyzed after the run. The offline analysis included changes in the analyzing power and the carbon energy from a more precise treatment of the silicon dead layer. The beam crossing timing as measured by the polarimeter waveform digitizers was found to vary by bunch number, and was determined for each bunch in the offline analysis. Carbon data were selected based on a 3 sigma cut on the reconstructed carbon mass, rather than using a fixed time window around the carbon locus in the time of flight vs. carbon energy distributions (banana plots).

It was found that, after these improvements to the analysis, the results were stable with different carbon selection cuts, a false asymmetry (cross asymmetry) that had appeared 
for some runs in the online was eliminated, and the asymmetry vs. $t$ behavior followed the analyzing power fit to the E950 data. The details of the systematic error estimation are described in the main chapters. 


\section{B. OFFLINE BLUE BEAM POLARIZATION RESULTS}

In each line of the table, following values are listed for each CNI measurement for blue beam. Note that offline values are available only for those runs measured with event mode.

fill RHIC fill number

run $\mathrm{CNI}$ run number within the fill

$\mathrm{P}$ polarization result without bunch selection

$\mathrm{dP}$ statistical error of $\mathrm{P}$

P_PH result with bunch selection customized for PHENIX (IP8)

dP_PH statistical error of P_PH

P_ST result with bunch selection customized for STAR (IP6)

dP_ST statistical error of P_ST Carbon energy spectrum)

$\begin{array}{lllllllll}\text { Fill } & \text { run } & \text { P } & \text { dP } & \text { P_PH } & \text { dP_PH } & \text { P_ST } & \text { dP_ST } \\ 3427 & 7 & -0.309 & 0.230 & -0.043 & 0.234 & -0.219 & 0.245 \\ 3476 & 2 & 0.201 & 0.046 & 0.225 & 0.048 & 0.204 & 0.049 \\ 3547 & 4 & 0.283 & 0.035 & 0.270 & 0.038 & 0.283 & 0.037 \\ 3547 & 5 & 0.334 & 0.035 & 0.335 & 0.038 & 0.317 & 0.037 \\ 3586 & 4 & 0.367 & 0.036 & 0.363 & 0.038 & 0.371 & 0.038 \\ 3602 & 2 & 0.372 & 0.036 & 0.382 & 0.038 & 0.374 & 0.038 \\ 3603 & 2 & 0.348 & 0.036 & 0.365 & 0.038 & 0.362 & 0.038 \\ 3604 & 2 & 0.331 & 0.036 & 0.319 & 0.038 & 0.352 & 0.038 \\ 3604 & 3 & 0.338 & 0.037 & 0.323 & 0.039 & 0.360 & 0.040 \\ 3606 & 3 & & 0.294 & 0.035 & 0.285 & 0.038 & 0.282 & 0.037 \\ 3606 & 4 & 0.294 & 0.035 & 0.316 & 0.038 & 0.327 & 0.037 \\ 3612 & 4 & 0.393 & 0.038 & 0.355 & 0.040 & 0.416 & 0.039 \\ 3612 & 5 & 0.343 & 0.035 & 0.336 & 0.038 & 0.347 & 0.037 \\ 3612 & 6 & 0.287 & 0.035 & 0.289 & 0.038 & 0.285 & 0.037 \\ 3613 & 3 & 0.328 & 0.036 & 0.324 & 0.038 & 0.311 & 0.038 \\ 3614 & 3 & 0.340 & 0.035 & 0.334 & 0.037 & 0.345 & 0.037 \\ 3614 & 4 & 0.316 & 0.035 & 0.322 & 0.037 & 0.317 & 0.038 \\ 3614 & 5 & 0.364 & 0.035 & 0.371 & 0.037 & 0.348 & 0.037 \\ 3615 & 2 & 0.241 & 0.036 & 0.220 & 0.038 & 0.241 & 0.038 \\ 3615 & 3 & & 0.271 & 0.035 & 0.286 & 0.037 & 0.253 & 0.038 \\ 3615 & 4 & 0.361 & 0.035 & 0.367 & 0.037 & 0.380 & 0.037 \\ 3620 & 2 & 0.316 & 0.036 & 0.303 & 0.038 & 0.329 & 0.038 \\ 3620 & 3 & 0.340 & 0.036 & 0.340 & 0.038 & 0.327 & 0.038 \\ 3620 & 4 & 0.298 & 0.036 & 0.308 & 0.038 & 0.312 & 0.038 \\ 3620 & 5 & 0.267 & 0.035 & 0.267 & 0.038 & 0.282 & 0.038 \\ 3622 & 2 & 0.281 & 0.036 & 0.280 & 0.039 & 0.285 & 0.039 \\ 3622 & 3 & 0.342 & 0.036 & 0.343 & 0.038 & 0.354 & 0.038 \\ 3624 & 4 & 0.366 & 0.036 & 0.356 & 0.038 & 0.392 & 0.038\end{array}$

$3627 \quad 2$

36302

36343

$3637 \quad 11$

$3640 \quad 3$

36442

36462

36464

$3654 \quad 1$

36542

36753

36764

$3677 \quad 4$

$3677 \quad 5$

36782

36793

$3680 \quad 4$

36805

36822

36843

36918

36963

36993

37023

37032

$3703 \quad 3$

$3705 \quad 3$

$3705 \quad 5$

37082

$3708 \quad 4$

$3708 \quad 6$

$3713 \quad 3$

37134

37135

37136

37137

$3714 \quad 6$

$3720 \quad 5$

37215

37217

37252

$3731 \quad 4$

37324

$3733 \quad 6$

37342

37343

$3735 \quad 3$

$3735 \quad 4$

$3751 \quad 2$

37512

$3757 \quad 3$

$3757 \quad 5$

37593

37594

37595

37642 $\begin{array}{llllll}0.261 & 0.041 & 0.257 & 0.044 & 0.258 & 0.044\end{array}$ $\begin{array}{lllllll}0.310 & 0.042 & 0.303 & 0.045 & 0.305 & 0.045\end{array}$ $\begin{array}{llllll}0.404 & 0.042 & 0.406 & 0.045 & 0.428 & 0.045\end{array}$ $\begin{array}{lllllll}0.288 & 0.042 & 0.263 & 0.045 & 0.304 & 0.045\end{array}$ $\begin{array}{lllllll}0.291 & 0.036 & 0.260 & 0.038 & 0.281 & 0.038\end{array}$ $\begin{array}{lllllll}0.412 & 0.047 & 0.407 & 0.051 & 0.420 & 0.051\end{array}$ $\begin{array}{llllll}0.363 & 0.036 & 0.368 & 0.038 & 0.356 & 0.038\end{array}$ $\begin{array}{llllll}0.317 & 0.036 & 0.298 & 0.038 & 0.328 & 0.038\end{array}$ $\begin{array}{llllll}0.263 & 0.037 & 0.251 & 0.040 & 0.273 & 0.039\end{array}$ $\begin{array}{llllll}0.273 & 0.037 & 0.260 & 0.040 & 0.257 & 0.039\end{array}$ $\begin{array}{lllllll}0.206 & 0.050 & 0.207 & 0.053 & 0.217 & 0.054\end{array}$ $\begin{array}{lllllll}0.285 & 0.036 & 0.291 & 0.039 & 0.275 & 0.038\end{array}$ $\begin{array}{llllll}0.291 & 0.037 & 0.303 & 0.039 & 0.271 & 0.039\end{array}$ $\begin{array}{llllll}0.301 & 0.037 & 0.299 & 0.039 & 0.289 & 0.039\end{array}$ $\begin{array}{lllllll}0.305 & 0.055 & 0.312 & 0.059 & 0.305 & 0.059\end{array}$ $\begin{array}{lllllll}0.324 & 0.039 & 0.339 & 0.042 & 0.337 & 0.041\end{array}$ $\begin{array}{lllllll}0.312 & 0.036 & 0.311 & 0.038 & 0.286 & 0.038\end{array}$ $\begin{array}{llllll}0.290 & 0.037 & 0.299 & 0.039 & 0.280 & 0.039\end{array}$ $\begin{array}{lllllll}0.311 & 0.038 & 0.304 & 0.041 & 0.285 & 0.040\end{array}$ $\begin{array}{lllllll}0.390 & 0.037 & 0.396 & 0.039 & 0.356 & 0.039\end{array}$ $\begin{array}{llllll}0.338 & 0.034 & 0.322 & 0.036 & 0.341 & 0.035\end{array}$ $\begin{array}{llllll}0.270 & 0.037 & 0.261 & 0.039 & 0.295 & 0.039\end{array}$ $\begin{array}{llllll}0.348 & 0.037 & 0.333 & 0.039 & 0.345 & 0.039\end{array}$ $\begin{array}{llllll}0.352 & 0.037 & 0.355 & 0.039 & 0.352 & 0.039\end{array}$ $\begin{array}{llllll}0.319 & 0.036 & 0.326 & 0.038 & 0.321 & 0.038\end{array}$ $\begin{array}{llllll}0.364 & 0.037 & 0.373 & 0.039 & 0.366 & 0.040\end{array}$ $\begin{array}{lllllll}0.354 & 0.037 & 0.343 & 0.039 & 0.354 & 0.039\end{array}$ $\begin{array}{llllll}0.320 & 0.036 & 0.335 & 0.039 & 0.299 & 0.038\end{array}$ $\begin{array}{llllll}0.300 & 0.036 & 0.303 & 0.038 & 0.287 & 0.038\end{array}$ $\begin{array}{lllllll}0.301 & 0.039 & 0.284 & 0.041 & 0.292 & 0.041\end{array}$ $\begin{array}{lllllll}0.331 & 0.036 & 0.332 & 0.038 & 0.328 & 0.038\end{array}$ $\begin{array}{llllll}0.343 & 0.036 & 0.324 & 0.038 & 0.353 & 0.038\end{array}$ $\begin{array}{llllll}0.342 & 0.036 & 0.336 & 0.038 & 0.329 & 0.038\end{array}$ $\begin{array}{lllllll}0.393 & 0.035 & 0.392 & 0.038 & 0.372 & 0.037\end{array}$ $\begin{array}{llllll}0.322 & 0.035 & 0.333 & 0.038 & 0.304 & 0.038\end{array}$ $\begin{array}{lllllll}0.276 & 0.035 & 0.276 & 0.038 & 0.267 & 0.037\end{array}$ $\begin{array}{llllll}0.340 & 0.035 & 0.329 & 0.038 & 0.346 & 0.038\end{array}$ $\begin{array}{lllllll}0.290 & 0.036 & 0.281 & 0.038 & 0.279 & 0.038\end{array}$ $\begin{array}{lllllll}0.312 & 0.036 & 0.299 & 0.039 & 0.312 & 0.039\end{array}$ $\begin{array}{lllllll}0.371 & 0.036 & 0.357 & 0.038 & 0.365 & 0.038\end{array}$ $\begin{array}{lllllll}0.336 & 0.036 & 0.306 & 0.038 & 0.324 & 0.038\end{array}$ $\begin{array}{lllllll}0.386 & 0.036 & 0.383 & 0.038 & 0.371 & 0.038\end{array}$ $\begin{array}{llllll}0.141 & 0.036 & 0.142 & 0.039 & 0.133 & 0.038\end{array}$ $\begin{array}{llllll}0.299 & 0.035 & 0.305 & 0.038 & 0.315 & 0.038\end{array}$ $\begin{array}{lllllll}0.341 & 0.036 & 0.329 & 0.038 & 0.368 & 0.038\end{array}$ $\begin{array}{llllll}0.294 & 0.037 & 0.331 & 0.040 & 0.296 & 0.040\end{array}$ $\begin{array}{llllll}0.343 & 0.036 & 0.356 & 0.038 & 0.335 & 0.038\end{array}$ $\begin{array}{llllll}0.400 & 0.045 & 0.377 & 0.048 & 0.425 & 0.047\end{array}$ $\begin{array}{lllllll}0.416 & 0.041 & 0.458 & 0.045 & 0.424 & 0.045\end{array}$ $\begin{array}{lllllll}0.416 & 0.041 & 0.458 & 0.045 & 0.424 & 0.045\end{array}$ $\begin{array}{llllll}0.376 & 0.040 & 0.381 & 0.043 & 0.391 & 0.043\end{array}$ $\begin{array}{llllll}0.375 & 0.040 & 0.371 & 0.043 & 0.365 & 0.043\end{array}$ $\begin{array}{lllllll}0.360 & 0.039 & 0.367 & 0.042 & 0.383 & 0.042\end{array}$ $\begin{array}{llllll}0.362 & 0.040 & 0.351 & 0.043 & 0.360 & 0.043\end{array}$ $\begin{array}{llllll}0.417 & 0.040 & 0.410 & 0.043 & 0.428 & 0.043\end{array}$ $\begin{array}{llllll}0.004 & 0.094 & -0.052 & 0.100 & -0.053 & 0.102\end{array}$ 
37643

37644

37645

37646

37653

37673

37693

37695

$3770 \quad 3$

$3774 \quad 3$

37793

$3780 \quad 3$

$3780 \quad 5$

$3780 \quad 7$

$3780 \quad 9$

$3780 \quad 11$

$3780 \quad 13$

$3780 \quad 15$

37843

37933

37936

37938

379310

37962

37963

$3797 \quad 3$

37992

37993

37994

$3799 \quad 5$

38014

$3803 \quad 3$

38035

38036

38037

38038

38039

38102 $\begin{array}{llllll}0.340 & 0.041 & 0.353 & 0.044 & 0.316 & 0.043\end{array}$ $\begin{array}{lllllll}0.435 & 0.040 & 0.445 & 0.043 & 0.429 & 0.043\end{array}$ $\begin{array}{lllllll}0.438 & 0.040 & 0.429 & 0.043 & 0.406 & 0.043\end{array}$ $\begin{array}{lllllll}0.371 & 0.040 & 0.369 & 0.043 & 0.356 & 0.043\end{array}$ $\begin{array}{lllllll}0.363 & 0.040 & 0.382 & 0.044 & 0.348 & 0.044\end{array}$ $\begin{array}{lllllll}0.227 & 0.040 & 0.226 & 0.043 & 0.205 & 0.043\end{array}$ $\begin{array}{llllll}0.296 & 0.049 & 0.278 & 0.052 & 0.337 & 0.052\end{array}$ $\begin{array}{lllllll}0.304 & 0.046 & 0.274 & 0.049 & 0.300 & 0.048\end{array}$ $\begin{array}{lllllll}0.354 & 0.045 & 0.368 & 0.047 & 0.367 & 0.047\end{array}$ $\begin{array}{llllll}0.150 & 0.049 & 0.139 & 0.052 & 0.176 & 0.052\end{array}$ $\begin{array}{lllllll}0.055 & 0.039 & 0.039 & 0.042 & 0.075 & 0.042\end{array}$ $\begin{array}{lllllll}0.308 & 0.042 & 0.286 & 0.044 & 0.299 & 0.044\end{array}$ $\begin{array}{lllllll}0.304 & 0.040 & 0.296 & 0.042 & 0.305 & 0.042\end{array}$ $\begin{array}{llllll}0.304 & 0.040 & 0.296 & 0.042 & 0.305 & 0.042\end{array}$ $\begin{array}{llllll}0.219 & 0.040 & 0.210 & 0.042 & 0.210 & 0.042\end{array}$ $\begin{array}{lllllll}0.290 & 0.039 & 0.260 & 0.042 & 0.304 & 0.042\end{array}$ $\begin{array}{lllllll}0.279 & 0.039 & 0.290 & 0.042 & 0.270 & 0.042\end{array}$ $\begin{array}{lllllll}0.280 & 0.039 & 0.288 & 0.042 & 0.279 & 0.041\end{array}$ $\begin{array}{lllllll}0.373 & 0.059 & 0.367 & 0.062 & 0.365 & 0.062\end{array}$ $\begin{array}{lllllll}0.216 & 0.059 & 0.188 & 0.062 & 0.247 & 0.062\end{array}$ $\begin{array}{lllllll}0.398 & 0.041 & 0.383 & 0.043 & 0.417 & 0.043\end{array}$ $\begin{array}{lllllll}0.280 & 0.041 & 0.266 & 0.043 & 0.292 & 0.043\end{array}$ $\begin{array}{lllllll}0.304 & 0.039 & 0.310 & 0.041 & 0.318 & 0.041\end{array}$ $\begin{array}{lllllll}0.279 & 0.039 & 0.289 & 0.041 & 0.284 & 0.042\end{array}$ $\begin{array}{lllllll}0.241 & 0.039 & 0.262 & 0.041 & 0.264 & 0.041\end{array}$ $\begin{array}{lllllll}0.417 & 0.039 & 0.408 & 0.042 & 0.411 & 0.042\end{array}$ $\begin{array}{lllllll}0.274 & 0.040 & 0.242 & 0.042 & 0.303 & 0.043\end{array}$ $\begin{array}{lllllll}0.213 & 0.039 & 0.189 & 0.042 & 0.232 & 0.042\end{array}$ $\begin{array}{lllllll}0.358 & 0.039 & 0.362 & 0.041 & 0.358 & 0.042\end{array}$ $\begin{array}{lllllll}0.261 & 0.039 & 0.263 & 0.041 & 0.249 & 0.041\end{array}$ $\begin{array}{lllllll}0.326 & 0.038 & 0.322 & 0.041 & 0.341 & 0.041\end{array}$ $\begin{array}{lllllll}0.202 & 0.043 & 0.173 & 0.046 & 0.194 & 0.046\end{array}$ $\begin{array}{lllllll}0.199 & 0.042 & 0.197 & 0.045 & 0.204 & 0.045\end{array}$ $\begin{array}{lllllll}0.280 & 0.041 & 0.238 & 0.044 & 0.277 & 0.044\end{array}$ $\begin{array}{lllllll}0.199 & 0.041 & 0.203 & 0.044 & 0.199 & 0.044\end{array}$ $\begin{array}{lllllll}0.204 & 0.041 & 0.186 & 0.043 & 0.212 & 0.043\end{array}$ $\begin{array}{lllllll}0.189 & 0.041 & 0.177 & 0.044 & 0.187 & 0.044\end{array}$ $\begin{array}{lllllll}0.194 & 0.042 & 0.177 & 0.044 & 0.192 & 0.044\end{array}$

\section{OFFLINE YELLOW BEAM POLARIZATION RESULTS}

The table below is the result of the offline analysis for yellow beam polarization. The definitions of the each column are the same as in blue.

Fill run P dP P_PH dP_PH P_ST dP_ST

$\begin{array}{llllllll}3427 & 115 & 0.058 & 0.044 & 0.095 & 0.048 & 0.044 & 0.047\end{array}$ $\begin{array}{llllllll}3476 & 102 & -0.008 & 0.029 & 0.024 & 0.031 & -0.017 & 0.031\end{array}$ $\begin{array}{llllllll}3547 & 105 & 0.323 & 0.028 & 0.340 & 0.031 & 0.329 & 0.030\end{array}$ $\begin{array}{llllllll}3547 & 106 & 0.269 & 0.028 & 0.296 & 0.031 & 0.253 & 0.030\end{array}$ $\begin{array}{llllllll}3586 & 102 & 0.345 & 0.028 & 0.350 & 0.030 & 0.342 & 0.030\end{array}$ $\begin{array}{llllllll}3602 & 102 & -0.008 & 0.029 & -0.004 & 0.031 & -0.011 & 0.030\end{array}$ $\begin{array}{llllllll}3603 & 102 & 0.012 & 0.028 & 0.017 & 0.030 & -0.004 & 0.030\end{array}$ $\begin{array}{llllllll}3604 & 102 & 0.279 & 0.029 & 0.278 & 0.031 & 0.290 & 0.031\end{array}$ $\begin{array}{lllllllll}3604 & 103 & 0.351 & 0.029 & 0.367 & 0.031 & 0.359 & 0.031\end{array}$ $\begin{array}{llllllll}3606 & 103 & 0.189 & 0.029 & 0.182 & 0.031 & 0.195 & 0.031\end{array}$ $\begin{array}{llllllll}3606 & 104 & 0.198 & 0.029 & 0.226 & 0.031 & 0.200 & 0.031\end{array}$ $\begin{array}{llllllll}3612 & 102 & 0.267 & 0.029 & 0.267 & 0.031 & 0.269 & 0.031\end{array}$ $\begin{array}{llllllll}3612 & 103 & 0.319 & 0.029 & 0.309 & 0.031 & 0.328 & 0.031\end{array}$ $\begin{array}{lllllllll}3612 & 104 & 0.281 & 0.029 & 0.272 & 0.031 & 0.286 & 0.031\end{array}$ $\begin{array}{llllllll}3612 & 105 & 0.320 & 0.029 & 0.303 & 0.031 & 0.349 & 0.030\end{array}$ $\begin{array}{llllllll}3612 & 106 & 0.259 & 0.029 & 0.273 & 0.031 & 0.255 & 0.031\end{array}$ $\begin{array}{lllllllll}3613 & 102 & 0.275 & 0.029 & 0.280 & 0.031 & 0.274 & 0.030\end{array}$ $\begin{array}{lllllllll}3613 & 103 & 0.231 & 0.029 & 0.239 & 0.031 & 0.220 & 0.031\end{array}$ $\begin{array}{lllllllll}3614 & 102 & 0.271 & 0.029 & 0.299 & 0.031 & 0.265 & 0.031\end{array}$ $\begin{array}{llllllll}3614 & 103 & 0.248 & 0.029 & 0.250 & 0.031 & 0.245 & 0.030\end{array}$ $\begin{array}{lllllllll}3614 & 104 & 0.334 & 0.029 & 0.337 & 0.031 & 0.343 & 0.030\end{array}$ $\begin{array}{lllllllll}3615 & 103 & 0.218 & 0.028 & 0.223 & 0.030 & 0.199 & 0.030\end{array}$ $\begin{array}{llllllll}3615 & 104 & 0.292 & 0.028 & 0.281 & 0.030 & 0.295 & 0.030\end{array}$ $\begin{array}{llllllll}3615 & 105 & 0.257 & 0.028 & 0.245 & 0.030 & 0.258 & 0.030\end{array}$ $\begin{array}{llllllll}3620 & 102 & 0.250 & 0.029 & 0.269 & 0.030 & 0.252 & 0.030\end{array}$ $\begin{array}{llllllll}3620 & 103 & 0.289 & 0.029 & 0.263 & 0.030 & 0.292 & 0.030\end{array}$ $\begin{array}{llllllll}3620 & 104 & 0.258 & 0.029 & 0.245 & 0.031 & 0.261 & 0.030\end{array}$ $\begin{array}{llllllll}3620 & 105 & 0.233 & 0.029 & 0.241 & 0.030 & 0.243 & 0.030\end{array}$ $\begin{array}{llllllll}3621 & 102 & 0.277 & 0.029 & 0.278 & 0.031 & 0.281 & 0.031\end{array}$ $\begin{array}{llllllll}3622 & 102 & 0.271 & 0.029 & 0.259 & 0.031 & 0.289 & 0.031\end{array}$ $\begin{array}{llllllll}3622 & 104 & 0.312 & 0.029 & 0.303 & 0.031 & 0.311 & 0.031\end{array}$ $\begin{array}{llllllll}3624 & 102 & 0.204 & 0.029 & 0.196 & 0.030 & 0.201 & 0.031\end{array}$ $\begin{array}{llllllll}3625 & 106 & 0.161 & 0.033 & 0.171 & 0.035 & 0.155 & 0.035\end{array}$ $\begin{array}{llllllll}3627 & 103 & 0.153 & 0.033 & 0.150 & 0.035 & 0.149 & 0.035\end{array}$ $\begin{array}{llllllll}3630 & 103 & 0.207 & 0.034 & 0.183 & 0.036 & 0.224 & 0.036\end{array}$ $\begin{array}{llllllll}3634 & 102 & 0.287 & 0.033 & 0.294 & 0.035 & 0.301 & 0.035\end{array}$ $\begin{array}{llllllll}3637 & 103 & 0.162 & 0.033 & 0.173 & 0.035 & 0.163 & 0.035\end{array}$ $\begin{array}{llllllll}3644 & 103 & 0.019 & 0.029 & 0.016 & 0.031 & 0.000 & 0.031\end{array}$ $\begin{array}{llllllll}3646 & 106 & 0.025 & 0.029 & 0.034 & 0.031 & 0.026 & 0.031\end{array}$ $\begin{array}{llllllll}3654 & 104 & 0.236 & 0.029 & 0.244 & 0.031 & 0.232 & 0.031\end{array}$ $\begin{array}{llllllll}3654 & 105 & 0.282 & 0.029 & 0.290 & 0.031 & 0.266 & 0.031\end{array}$ $\begin{array}{llllllll}3672 & 103 & 0.264 & 0.029 & 0.253 & 0.031 & 0.275 & 0.031\end{array}$ $\begin{array}{llllllll}3675 & 105 & 0.231 & 0.028 & 0.239 & 0.030 & 0.228 & 0.030\end{array}$ $\begin{array}{llllllll}3676 & 105 & 0.151 & 0.028 & 0.145 & 0.030 & 0.161 & 0.030\end{array}$ $\begin{array}{llllllll}3677 & 104 & 0.239 & 0.040 & 0.248 & 0.043 & 0.253 & 0.043\end{array}$ $\begin{array}{llllllll}3677 & 105 & 0.271 & 0.028 & 0.263 & 0.030 & 0.276 & 0.030\end{array}$ $\begin{array}{llllllll}3677 & 106 & 0.182 & 0.028 & 0.184 & 0.030 & 0.177 & 0.030\end{array}$ $\begin{array}{llllllll}3677 & 108 & 0.218 & 0.029 & 0.227 & 0.031 & 0.223 & 0.031\end{array}$ $\begin{array}{llllllll}3678 & 103 & 0.322 & 0.028 & 0.310 & 0.031 & 0.339 & 0.031\end{array}$ $\begin{array}{llllllll}3679 & 104 & 0.211 & 0.028 & 0.205 & 0.031 & 0.218 & 0.031\end{array}$ $\begin{array}{llllllll}3680 & 105 & 0.155 & 0.027 & 0.158 & 0.030 & 0.147 & 0.029\end{array}$ $\begin{array}{llllllll}3682 & 102 & 0.224 & 0.028 & 0.232 & 0.031 & 0.235 & 0.031\end{array}$ $\begin{array}{llllllll}3684 & 103 & 0.081 & 0.028 & 0.101 & 0.030 & 0.086 & 0.031\end{array}$ $\begin{array}{llllllll}3691 & 107 & 0.311 & 0.028 & 0.334 & 0.030 & 0.329 & 0.030\end{array}$ $\begin{array}{llllllll}3698 & 106 & 0.136 & 0.038 & 0.169 & 0.041 & 0.148 & 0.040\end{array}$ $\begin{array}{llllllll}3699 & 102 & 0.311 & 0.029 & 0.316 & 0.031 & 0.316 & 0.031\end{array}$ $\begin{array}{llllllll}3699 & 104 & 0.157 & 0.028 & 0.171 & 0.030 & 0.143 & 0.030\end{array}$ $\begin{array}{llllllll}3702 & 102 & 0.219 & 0.028 & 0.215 & 0.030 & 0.231 & 0.030\end{array}$ $\begin{array}{llllllll}3703 & 102 & 0.142 & 0.029 & 0.151 & 0.031 & 0.158 & 0.031\end{array}$ $\begin{array}{llllllll}3705 & 103 & 0.286 & 0.028 & 0.288 & 0.030 & 0.284 & 0.030\end{array}$ $\begin{array}{llllllll}3705 & 104 & 0.317 & 0.029 & 0.336 & 0.031 & 0.310 & 0.031\end{array}$ $\begin{array}{llllllll}3708 & 103 & 0.347 & 0.029 & 0.327 & 0.031 & 0.350 & 0.031\end{array}$ 
$\begin{array}{llllllll}3708 & 105 & 0.236 & 0.028 & 0.263 & 0.031 & 0.227 & 0.031\end{array}$

$\begin{array}{llllllll}3708 & 107 & 0.136 & 0.065 & 0.126 & 0.070 & 0.116 & 0.069\end{array}$

$\begin{array}{llllllll}3713 & 102 & 0.223 & 0.028 & 0.231 & 0.030 & 0.228 & 0.030\end{array}$

$\begin{array}{llllllll}3713 & 104 & 0.327 & 0.028 & 0.329 & 0.030 & 0.322 & 0.030\end{array}$

$\begin{array}{lllllllll}3713 & 105 & 0.322 & 0.028 & 0.333 & 0.030 & 0.326 & 0.030\end{array}$

$\begin{array}{llllllll}3713 & 108 & 0.246 & 0.030 & 0.241 & 0.032 & 0.233 & 0.032\end{array}$

$\begin{array}{llllllll}3714 & 105 & 0.302 & 0.028 & 0.320 & 0.030 & 0.302 & 0.030\end{array}$

$\begin{array}{llllllll}3720 & 108 & 0.275 & 0.031 & 0.279 & 0.033 & 0.270 & 0.033\end{array}$

$\begin{array}{llllllll}3720 & 110 & 0.300 & 0.031 & 0.299 & 0.033 & 0.324 & 0.033\end{array}$

$\begin{array}{lllllllll}3721 & 102 & 0.253 & 0.031 & 0.248 & 0.034 & 0.270 & 0.034\end{array}$

$\begin{array}{llllllll}3721 & 105 & 0.269 & 0.031 & 0.270 & 0.033 & 0.285 & 0.034\end{array}$

$\begin{array}{llllllll}3721 & 107 & 0.285 & 0.031 & 0.292 & 0.033 & 0.275 & 0.034\end{array}$

$\begin{array}{llllllll}3731 & 103 & 0.298 & 0.031 & 0.286 & 0.033 & 0.304 & 0.033\end{array}$

$\begin{array}{llllllll}3733 & 105 & 0.269 & 0.031 & 0.268 & 0.034 & 0.256 & 0.034\end{array}$

$\begin{array}{lllllllll}3734 & 102 & 0.231 & 0.031 & 0.203 & 0.033 & 0.242 & 0.034\end{array}$

$\begin{array}{llllllll}3734 & 103 & 0.268 & 0.031 & 0.259 & 0.033 & 0.242 & 0.033\end{array}$

$\begin{array}{llllllll}3735 & 103 & 0.170 & 0.031 & 0.169 & 0.033 & 0.189 & 0.034\end{array}$

$\begin{array}{llllllll}3735 & 104 & 0.242 & 0.031 & 0.254 & 0.033 & 0.247 & 0.033\end{array}$

$\begin{array}{lllllllll}3756 & 102 & 0.321 & 0.055 & 0.376 & 0.059 & 0.364 & 0.058\end{array}$

$\begin{array}{lllllllll}3756 & 103 & 0.293 & 0.045 & 0.401 & 0.048 & 0.366 & 0.047\end{array}$

$\begin{array}{lllllllll}3757 & 103 & 0.335 & 0.037 & 0.376 & 0.039 & 0.369 & 0.039\end{array}$

$\begin{array}{llllllll}3757 & 105 & 0.328 & 0.039 & 0.326 & 0.041 & 0.318 & 0.041\end{array}$

$\begin{array}{lllllllll}3780 & 103 & 0.245 & 0.034 & 0.239 & 0.036 & 0.230 & 0.036\end{array}$

$\begin{array}{lllllllll}3780 & 105 & 0.231 & 0.034 & 0.237 & 0.036 & 0.235 & 0.036\end{array}$

$\begin{array}{lllllllll}3780 & 107 & 0.231 & 0.034 & 0.237 & 0.036 & 0.235 & 0.036\end{array}$

$\begin{array}{lllllllll}3780 & 108 & 0.238 & 0.033 & 0.241 & 0.035 & 0.240 & 0.035\end{array}$

$\begin{array}{llllllll}3780 & 110 & 0.240 & 0.033 & 0.226 & 0.036 & 0.233 & 0.035\end{array}$

$\begin{array}{lllllllll}3780 & 112 & 0.215 & 0.033 & 0.227 & 0.036 & 0.211 & 0.036\end{array}$

$\begin{array}{lllllllll}3780 & 114 & 0.264 & 0.033 & 0.283 & 0.036 & 0.264 & 0.036\end{array}$

$\begin{array}{llllllll}3780 & 116 & 0.173 & 0.033 & 0.172 & 0.036 & 0.197 & 0.036\end{array}$

$\begin{array}{llllllll}3784 & 102 & 0.262 & 0.035 & 0.294 & 0.038 & 0.253 & 0.038\end{array}$

$\begin{array}{llllllll}3793 & 103 & 0.265 & 0.048 & 0.273 & 0.051 & 0.241 & 0.051\end{array}$

$\begin{array}{lllllllll}3793 & 105 & 0.245 & 0.034 & 0.225 & 0.036 & 0.241 & 0.036\end{array}$

$\begin{array}{llllllll}3793 & 107 & 0.195 & 0.034 & 0.198 & 0.036 & 0.182 & 0.036\end{array}$

$\begin{array}{llllllll}3793 & 109 & 0.186 & 0.033 & 0.186 & 0.035 & 0.201 & 0.035\end{array}$

$\begin{array}{lllllllll}3796 & 104 & 0.195 & 0.032 & 0.197 & 0.034 & 0.187 & 0.034\end{array}$

$\begin{array}{lllllllll}3797 & 104 & 0.220 & 0.032 & 0.217 & 0.034 & 0.229 & 0.034\end{array}$

$\begin{array}{lllllllll}3799 & 102 & 0.296 & 0.032 & 0.318 & 0.034 & 0.304 & 0.034\end{array}$

$\begin{array}{llllllll}3799 & 103 & 0.280 & 0.032 & 0.283 & 0.034 & 0.264 & 0.034\end{array}$

$\begin{array}{llllllll}3799 & 104 & 0.260 & 0.032 & 0.271 & 0.034 & 0.254 & 0.034\end{array}$

$\begin{array}{lllllllll}3801 & 104 & 0.250 & 0.033 & 0.246 & 0.035 & 0.274 & 0.035\end{array}$

$\begin{array}{llllllll}3803 & 103 & 0.302 & 0.034 & 0.281 & 0.036 & 0.310 & 0.036\end{array}$

$\begin{array}{llllllll}3803 & 104 & 0.249 & 0.033 & 0.244 & 0.035 & 0.244 & 0.035\end{array}$

$\begin{array}{llllllll}3803 & 105 & 0.251 & 0.033 & 0.235 & 0.035 & 0.241 & 0.035\end{array}$

$\begin{array}{lllllllll}3803 & 106 & 0.240 & 0.033 & 0.237 & 0.036 & 0.250 & 0.036\end{array}$

$\begin{array}{lllllllll}3803 & 107 & 0.289 & 0.033 & 0.296 & 0.036 & 0.276 & 0.036\end{array}$

$\begin{array}{lllllllll}3803 & 112 & 0.368 & 0.066 & 0.366 & 0.071 & 0.377 & 0.070\end{array}$

$\begin{array}{llllllll}3810 & 102 & 0.126 & 0.034 & 0.134 & 0.037 & 0.130 & 0.037\end{array}$

$\begin{array}{llllllll}3810 & 102 & 0.126 & 0.034 & 0.134 & 0.037 & 0.130 & 0.037\end{array}$

\section{ONLINE (SCALER) POLARIZATION RESULTS}

These results are for Run 3, and are based on the scaler polarimeter information which was also distributed to experiments through CDEV during the run period. The analyzing power used is an effective analyzing power from a fit to the E950 data, weighted by the observed carbon energy distribution. In the offline analysis, the fit was corrected, and the carbon energy values changed, due to a more precise treatment of the silicon dead layer. These corrections changed the offline polarization values relative to the online values by $\mathrm{dP} / \mathrm{P}=+0.09$ for blue and +0.05 for yellow both at flattop (blue: +0.16 , yellw: +0.01 for injection). The polarization values for the run should be taken from the event mode values (appendices above), except when event mode data are not available. In that case, the scaler values below can be used, corrected by the factor above.

Fill.run Beam Energy date time $P$ dP

3407.005 24.3 Apr 7 3407.006 24.3 Apr 7 3407.007 24.3 Apr 7 3407.008 24.3 Apr 7 3407.00924 .3 Apr 7 3407.010 24.3 Apr 7 3407.01124 .3 Apr 7 3407.10124 .3 Apr 7 3407.102 24.3 Apr 7 3407.103 24.3 Apr 7 3408.002 24.3 Apr 7 3408.003 24.3 Apr 7 3408.101 24.3 Apr 7 3408.102 24.3 Apr 7 3408.103 24.3 Apr 7 3420.00124 .3 Apr 8 3420.002 24.3 Apr 8 3420.10124 .3 Apr 8 3420.102 24.3 Apr 8 3424.00199 .8 Apr 8 3424.00399 .8 Apr 8 3424.00499 .8 Apr 8 3424.10199 .8 Apr 8 3424.10299 .8 Apr 8 3425.00399 .8 Apr 8 3425.10199 .8 Apr 8 3427.001 24.3 Apr 8 3427.002 24.3 Apr 8 3427.00399 .8 Apr 8 3427.00499 .8 Apr 8 3427.00599 .8 Apr 8 3427.00699 .8 Apr 8 3427.007 99.8 Apr 8
03:25:48

03:30:04

03:34:01

03:38:39

03:45:31

03:51:43

03:56:00

04:20:20

04:21:45

04:28:05

05:55:46

06:53:50

06:19:17

06:30:01

07:07:07

04:34:52

05:32:11

$04: 44: 26$

05:23:49

11:15:19

11:44:30

12:09:18

$12: 26: 47$

12:36:46

15:04:59

$14: 49: 46$

16:40:05

$16: 42: 12$

16:53:44

17:00:20

18:23:53

18:30:28

18:53:48 $\begin{array}{lll}-0.32049 & 0.02753\end{array}$ $\begin{array}{lll}-0.34853 & 0.02840\end{array}$ $\begin{array}{ll}-0.29483 & 0.02901\end{array}$ $\begin{array}{lll}-0.32903 & 0.02950\end{array}$ $\begin{array}{lll}-0.27227 & 0.03663\end{array}$ $\begin{array}{lll}-0.31629 & 0.02917\end{array}$ $\begin{array}{lll}-0.30015 & 0.01891\end{array}$ $\begin{array}{lll}0.06922 & 0.04836\end{array}$ $\begin{array}{lll}-0.01567 & 0.01938\end{array}$ $\begin{array}{lll}-0.00944 & 0.01931\end{array}$ $\begin{array}{lll}0.34829 & 0.04030\end{array}$ $\begin{array}{lll}0.31157 & 0.02026\end{array}$ $\begin{array}{ll}0.12177 & 0.03368\end{array}$ $\begin{array}{lll}0.01084 & 0.04448\end{array}$ $\begin{array}{lll}-0.04177 & 0.01935\end{array}$ $\begin{array}{lll}0.39080 & 0.03646\end{array}$ $\begin{array}{lll}-0.01677 & 0.03762\end{array}$ $\begin{array}{lll}0.01182 & 0.02455\end{array}$ $\begin{array}{lll}-0.26656 & 0.02179\end{array}$ $\begin{array}{lll}0.44608 & 0.03805\end{array}$ $\begin{array}{ll}0.32839 & 0.03529\end{array}$ $\begin{array}{ll}0.26077 & 0.03522\end{array}$ $\begin{array}{ll}0.19366 & 0.02725\end{array}$ $\begin{array}{lll}0.15239 & 0.02861\end{array}$ $\begin{array}{lll}0.15506 & 0.02768\end{array}$ $\begin{array}{lll}-0.03924 & 0.03240\end{array}$ $\begin{array}{lll}0.36054 & 0.03985\end{array}$ $\begin{array}{lll}0.34450 & 0.01816\end{array}$ $\begin{array}{lll}0.35343 & 0.01757\end{array}$ $\begin{array}{ll}0.35452 & 0.01798\end{array}$ $\begin{array}{ll}0.35042 & 0.01766\end{array}$ $\begin{array}{ll}0.30182 & 0.03650\end{array}$ $0.30672 \quad 0.02516$ 
3427.101 24.3 Apr 8 3427.102 24.3 Apr 8 3427.109 99.8 Apr 8 3427.11099 .8 Apr 8 3427.11199 .8 Apr 8 3427.113 99.8 Apr 8 3427.114 99.8 Apr 8 3427.115 99.8 Apr 8 3441.101 24.3 Apr 10 3451.101 24.3 Apr 11 3459.002 24.3 Apr 12 3459.003 99.8 Apr 12 3459.005 99.8 Apr 12 3459.006 99.8 Apr 12 3459.007 99.8 Apr 12 3459.101 24.3 Apr 12 3459.102 99.8 Apr 12 3459.103 99.8 Apr 12 3459.104 99.8 Apr 12 3459.105 99.8 Apr 12 3459.106 99.8 Apr 12 3459.107 99.8 Apr 12 3460.002 24.3 Apr 12 3460.101 24.3 Apr 12 3460.102 24.3 Apr 12 3461.00124 .3 Apr 12 3461.101 24.3 Apr 12 3467.001 24.3 Apr 13 3467.101 24.3 Apr 13 3468.001 24.3 Apr 13 3468.002 99.8 Apr 13 3468.101 24.3 Apr 13 3468.102 99.8 Apr 13 3468.103 99.8 Apr 13 3470.001 24.3 Apr 13 3470.101 24.3 Apr 13 3471.001 24.3 Apr 13 3471.003 24.3 Apr 13 3471.00499 .8 Apr 13 3471.005 99.8 Apr 13 3471.006 99.8 Apr 13 3471.101 24.3 Apr 13 3471.102 24.3 Apr 13 3471.103 99.8 Apr 13 3471.10499 .8 Apr 13 3471.105 99.8 Apr 13 3472.002 99.8 Apr 13 3472.101 24.3 Apr 13 3472.102 99.8 Apr 13 3472.103 99.8 Apr 13 3473.001 24.3 Apr 13 3473.101 24.3 Apr 13 3474.001 24.3 Apr 13 3474.002 99.8 Apr 13 3474.101 24.3 Apr 13 3475.001 24.3 Apr 13
$16: 20: 29$

$16: 33: 52$

$17: 54: 47$

$17: 57: 04$

18:00:53

$18: 38: 45$

$18: 42: 18$

19:00:46

$15: 34: 13$

$11: 46: 44$

01:05:37

01:18:30

06:06:32

08:33:30

$09: 29: 29$

$00: 57: 53$

$01: 23: 28$

$03: 56: 17$

06:02:42

$06: 20: 37$

08:30:16

$09: 26: 15$

11:50:07

$11: 32: 30$

$11: 40: 11$

$15: 37: 02$

$15: 54: 17$

$02: 33: 18$

$02: 25: 22$

03:34:04

$03: 51: 37$

$03: 21: 46$

03:49:48

03:56:45

$07: 13: 42$

07:06:43

08:55:17

09:33:39

10:09:01

12:02:25

$13: 46: 10$

09:07:51

09:23:12

10:07:38

$12: 00: 55$

$13: 44: 06$

15:29:08

$14: 21: 46$

$15: 27: 01$

$15: 46: 34$

19:03:53

$18: 52: 21$

$20: 22: 58$

20:39:50

20:02:52

22:45:53 $\begin{array}{ll}0.39277 & 0.01946\end{array}$

$\begin{array}{ll}0.37078 & 0.01951\end{array}$

$\begin{array}{ll}0.00154 & 0.03157\end{array}$

$\begin{array}{ll}0.02240 & 0.01959\end{array}$

$0.00084 \quad 0.01966$

$\begin{array}{ll}0.05235 & 0.03836\end{array}$

$\begin{array}{lll}-0.05733 & 0.03664\end{array}$

$\begin{array}{ll}0.02520 & 0.02618\end{array}$

$\begin{array}{ll}0.07405 & 0.06374\end{array}$

$0.43814 \quad 0.04876$

$\begin{array}{ll}0.30254 & 0.02315\end{array}$

$0.23082 \quad 0.02383$

$0.25005 \quad 0.02192$

$0.24827 \quad 0.02201$

$0.22981 \quad 0.02193$

0.312620 .02433

$0.12095 \quad 0.02423$

$\begin{array}{ll}0.12076 & 0.02272\end{array}$

$\begin{array}{ll}0.03455 & 0.02252\end{array}$

$0.05866 \quad 0.02257$

$0.06850 \quad 0.02264$

$0.08347 \quad 0.02247$

$\begin{array}{ll}0.03391 & 0.02366\end{array}$

$\begin{array}{ll}1.32242 & 0.02412\end{array}$

$\begin{array}{ll}1.37318 & 0.04968\end{array}$

$\begin{array}{ll}0.23906 & 0.03541\end{array}$

$\begin{array}{ll}0.35528 & 0.02728\end{array}$

$\begin{array}{ll}0.28341 & 0.02507\end{array}$

0.340640 .02402

$0.29804 \quad 0.02526$

$\begin{array}{ll}0.27439 & 0.02454\end{array}$

$0.36223 \quad 0.02367$

$0.22218 \quad 0.02397$

$\begin{array}{ll}0.18232 & 0.02386\end{array}$

$\begin{array}{ll}-0.03391 & 0.02526\end{array}$

$\begin{array}{lll}0.33796 & 0.02413\end{array}$

$\begin{array}{lll}0.03158 & 0.02445\end{array}$

$0.26852 \quad 0.02525$

$\begin{array}{ll}0.17892 & 0.02466\end{array}$

$\begin{array}{ll}0.16085 & 0.02365\end{array}$

0.183640 .02385

$\begin{array}{ll}-0.04195 & 0.02399\end{array}$

$\begin{array}{ll}0.38057 & 0.02397\end{array}$

$0.17755 \quad 0.02407$

$0.15974 \quad 0.02398$

$\begin{array}{ll}-0.00378 & 0.02417\end{array}$

$\begin{array}{ll}0.34052 & 0.02385\end{array}$

$0.32135 \quad 0.02416$

$0.01127 \quad 0.02442$

$0.00404 \quad 0.02422$

$\begin{array}{ll}0.36343 & 0.02450\end{array}$

$\begin{array}{ll}0.38423 & 0.02440\end{array}$

$0.31546 \quad 0.02444$

$\begin{array}{ll}0.23422 & 0.02414\end{array}$

$\begin{array}{ll}0.36586 & 0.02430\end{array}$

$\begin{array}{ll}-0.16822 & 0.02445\end{array}$
3475.002 99.8 Apr 13 3475.10124 .3 Apr 13 3475.102 24.3 Apr 13 3476.001 24.3 Apr 14 3476.00299 .8 Apr 14 3476.10124 .3 Apr 14 3476.102 99.8 Apr 14 3480.001 24.3 Apr 14 3480.002 24.3 Apr 14 3480.00499 .8 Apr 15 3480.101 24.3 Apr 14 3480.102 24.3 Apr 14 3480.10399 .8 Apr 14 3480.10499 .8 Apr 15 3502.001 99.8 Apr 19 3502.00299 .8 Apr 19 3502.102 99.8 Apr 19 3503.001 24.3 Apr 19 3503.002 24.3 Apr 19 3503.003 99.8 Apr 19 3503.00499 .8 Apr 19 3504.00124 .3 Apr 19 3504.002 24.3 Apr 19 3505.001 24.3 Apr 19 3505.002 24.3 Apr 19 3505.101 24.3 Apr 19 3505.102 24.3 Apr 19 3506.001 99.8 Apr 19 3506.10199 .8 Apr 19 3508.001 24.3 Apr 20 3508.00299 .8 Apr 20 3508.003 99.8 Apr 20 3508.10124 .3 Apr 20 3508.102 99.8 Apr 20 3508.10399 .8 Apr 20 3509.00199 .8 Apr 20 3509.10199 .8 Apr 20 3510.001 24.3 Apr 20 3510.00299 .8 Apr 20 3510.10124 .3 Apr 20 3510.10299 .8 Apr 20 3517.102 24.3 Apr 20 3518.00199 .8 Apr 21 3518.10199 .8 Apr 21 3521.00199 .8 Apr 21 3521.00299 .8 Apr 21 3521.10199 .8 Apr 21 3523.00199 .8 Apr 21 3523.10399 .8 Apr 21 3523.105 99.8 Apr 21 3524.001 24.3 Apr 21 3524.00299 .8 Apr 21 3524.00399 .8 Apr 21 3524.00499 .8 Apr 22 3524.00599 .8 Apr 22 3524.101 24.3 Apr 21
$23: 36: 41$ $22: 38: 25$ $22: 40: 33$ 02:16:35 02:31:05 02:10:23 $02: 37: 48$ 20:26:38 22:55:09 02:15:10 20:17:52 22:39:19 23:41:00 $02: 44: 23$ 09:11:30 $09: 24: 58$ 09:34:59 12:13:03 $12: 26: 46$ 13:56:07 $14: 05: 16$ 15:01:50 15:08:16 19:20:37 $19: 29: 53$ 18:04:40

19:11:17 21:59:27 21:52:21 02:07:38

02:59:57

09:25:09

$01: 47: 56$

02:53:34

09:17:46

12:27:07

12:20:01

15:09:21

$15: 22: 46$

15:01:20

$15: 21: 10$

23:47:07

01:13:43

$01: 21: 29$

$06: 12: 48$

14:02:27

06:06:15

15:46:25

$15: 35: 28$

15:41:01

20:59:11

21:31:35 $22: 38: 59$

02:01:10

03:58:25

20:53:44

$\begin{array}{ll}0.03341 & 0.02307\end{array}$ $\begin{array}{lll}-0.03872 & 0.02413\end{array}$ $\begin{array}{ll}-0.00412 & 0.02435\end{array}$ $\begin{array}{ll}0.36046 & 0.02255\end{array}$ $\begin{array}{lll}0.24216 & 0.02284\end{array}$ $\begin{array}{ll}0.36734 & 0.02431\end{array}$ $\begin{array}{ll}-0.00112 & 0.02419\end{array}$ $\begin{array}{ll}0.29048 & 0.02930\end{array}$ 
3524.102 99.8 Apr 21 3524.103 99.8 Apr 21 3526.00199 .8 Apr 22 3526.00299 .8 Apr 22 3526.003 99.8 Apr 22 3526.101 24.3 Apr 22 3526.102 99.8 Apr 22 3526.103 99.8 Apr 22 3526.104 99.8 Apr 22 3526.105 99.8 Apr 22 3527.002 99.8 Apr 22 3527.102 24.3 Apr 22 3527.103 99.8 Apr 22 3527.10499 .8 Apr 22 3539.013 24.3 Apr 24 3539.014 99.8 Apr 24 3539.015 99.8 Apr 24 3539.01699 .8 Apr 24 3539.017 99.8 Apr 24 3539.112 24.3 Apr 24 3539.113 99.8 Apr 24 3539.114 99.8 Apr 24 3539.115 99.8 Apr 24 3540.00199 .8 Apr 24 3540.002 99.8 Apr 24 3540.101 99.8 Apr 24 3540.102 99.8 Apr 24 3545.002 99.8 Apr 25 3545.003 99.8 Apr 25 3545.10124 .3 Apr 25 3545.102 24.3 Apr 25 3545.103 24.3 Apr 25 3545.104 99.8 Apr 25 3545.105 99.8 Apr 25 3547.00199 .8 Apr 25 3547.002 99.8 Apr 25 3547.003 99.8 Apr 25 3547.004 99.8 Apr 25 3547.005 99.8 Apr 26 3547.006 99.8 Apr 26 3547.007 99.8 Apr 26 3547.00899 .8 Apr 26 3547.101 99.8 Apr 25 3547.103 99.8 Apr 25 3547.104 99.8 Apr 25 3547.105 99.8 Apr 25 3547.10699 .8 Apr 26 3547.107 99.8 Apr 26 3547.108 99.8 Apr 26 3547.109 99.8 Apr 26 3547.110 99.8 Apr 26 3549.002 24.3 Apr 26 3549.013 24.3 Apr 26 3549.01499 .8 Apr 26 3549.103 24.3 Apr 26 3549.105 24.3 Apr 26
$21: 29: 58$ $22: 32: 41$ $17: 28: 55$ 18:02:33 $19: 34: 16$ 17:00:45 $17: 23: 49$ 18:07:46 $19: 20: 19$ $19: 26: 54$ 22:00:27 21:04:21 21:28:07 21:53:08 19:30:04 20:04:36 $20: 28: 53$ $21: 20: 30$ $21: 32: 13$ $19: 35: 52$ 20:10:09 $20: 35: 19$ $21: 26: 07$ $22: 32: 47$ 22:59:24 $22: 27: 31$ 22:52:28 13:06:01 $13: 27: 12$ $10: 54: 57$ $12: 25: 55$ $12: 35: 21$ $12: 58: 54$ $13: 21: 04$ $18: 49: 26$ 19:03:42 19:40:18 $21: 58: 52$ 00:56:08 04:09:09 07:08:52 09:29:31

$18: 47: 37$ 19:02:08 19:39:08 21:52:11 00:50:39 04:06:31 $07: 16: 18$ $09: 35: 59$ 11:48:39 22:09:41 23:11:06 $23: 25: 55$ $17: 17: 18$ $18: 23: 41$ $\begin{array}{ll}-0.01537 & 0.02383\end{array}$ $\begin{array}{ll}-0.01739 & 0.02387\end{array}$ $0.36815 \quad 0.04506$ $0.19271 \quad 0.04640$ $0.17768 \quad 0.05045$ $0.35502 \quad 0.04050$ $0.11931 \quad 0.03456$ $-0.00802 \quad 0.03261$ $\begin{array}{ll}0.00572 & 0.04623\end{array}$ $\begin{array}{ll}-0.01723 & 0.03838\end{array}$ $\begin{array}{ll}0.25098 & 0.05131\end{array}$ $0.39765 \quad 0.03713$ $0.10755 \quad 0.03343$ $\begin{array}{ll}0.02618 & 0.03770\end{array}$ $0.43118 \quad 0.02741$ $\begin{array}{ll}0.32135 & 0.02970\end{array}$ $0.42480 \quad 0.04203$ $\begin{array}{ll}0.33081 & 0.04076\end{array}$ $0.32421 \quad 0.04220$ $0.41435 \quad 0.02411$ $\begin{array}{ll}-0.02936 & 0.03064\end{array}$ $\begin{array}{ll}-0.00443 & 0.03266\end{array}$ $\begin{array}{ll}0.04479 & 0.05263\end{array}$ $0.27568 \quad 0.04962$ $0.36181 \quad 0.02446$ $\begin{array}{ll}-0.01392 & 0.02450\end{array}$ $\begin{array}{ll}-0.02970 & 0.02552\end{array}$ $\begin{array}{ll}0.30391 & 0.02993\end{array}$ $\begin{array}{ll}0.28022 & 0.03991\end{array}$ $\begin{array}{ll}-0.03738 & 0.03977\end{array}$ $\begin{array}{ll}0.42028 & 0.03902\end{array}$ $\begin{array}{ll}0.38964 & 0.03430\end{array}$ $0.25028 \quad 0.02451$ $\begin{array}{ll}-0.03062 & 0.02866\end{array}$ $0.42194 \quad 0.02225$ $\begin{array}{ll}0.30956 & 0.02264\end{array}$ $0.35948 \quad 0.02231$ $\begin{array}{ll}0.31863 & 0.02283\end{array}$ $\begin{array}{ll}0.34255 & 0.02260\end{array}$ $\begin{array}{ll}0.34765 & 0.02278\end{array}$ $0.37223 \quad 0.02253$ $0.34128 \quad 0.02263$ $\begin{array}{ll}0.34034 & 0.02383\end{array}$ $\begin{array}{ll}0.28943 & 0.02388\end{array}$ $0.31964 \quad 0.02379$ $\begin{array}{ll}0.31791 & 0.02388\end{array}$ $0.24138 \quad 0.02369$ $\begin{array}{ll}0.23031 & 0.02387\end{array}$ $0.27032 \quad 0.02388$ $\begin{array}{ll}0.23040 & 0.02379\end{array}$ 0.268890 .02380 $\begin{array}{ll}0.13328 & 0.02731\end{array}$ $0.43966 \quad 0.02243$ $0.30611 \quad 0.02364$ $\begin{array}{ll}0.44757 & 0.04172\end{array}$ $0.37751 \quad 0.02367$
3549.10624 .3 Apr 26 3549.107 24.3 Apr 26 3549.110 24.3 Apr 26 3549.11124 .3 Apr 26 3549.112 24.3 Apr 26 3549.11399 .8 Apr 26 3550.00424 .3 Apr 27 3551.10124 .3 Apr 27 3552.001 24.3 Apr 27 3552.10124 .3 Apr 27 3552.102 99.8 Apr 27 3553.00124 .3 Apr 27 3553.101 24.3 Apr 27 3556.00199 .8 Apr 27 3563.00199 .8 Apr 28 3563.00299 .8 Apr 28 3563.003 99.8 Apr 28 3563.00499 .8 Apr 28 3563.10199 .8 Apr 28 3568.10199 .8 Apr 28 3569.00199 .8 Apr 28 3569.10199 .8 Apr 28 3569.102 99.8 Apr 28 3571.00199 .8 Apr 28 3572.00199 .8 Apr 28 3572.10124 .3 Apr 28 3572.102 99.8 Apr 28 3573.002 24.3 Apr 28 3573.10124 .3 Apr 28 3574.00199 .8 Apr 28 3574.00299 .8 Apr 28 3574.10199 .8 Apr 28 3574.102 99.8 Apr 28 3575.00599 .8 Apr 29 3575.10124 .3 Apr 28 3575.102 99.8 Apr 29 3575.103 99.8 Apr 29 3576.001 24.3 Apr 29 3576.10124 .3 Apr 29 3578.00124 .3 Apr 29 3578.10124 .3 Apr 29 3578.102 24.3 Apr 29 3580.00124 .3 Apr 29 3580.00299 .8 Apr 29 3580.00399 .8 Apr 29 3580.101 24.3 Apr 29 3580.10299 .8 Apr 29 3580.10399 .8 Apr 29 3582.00124 .3 Apr 29 3582.002 24.3 Apr 29 3582.003 24.3 Apr 29 3582.00499 .8 Apr 29 3583.001 24.3 Apr 29 3583.002 99.8 Apr 29 3583.003 99.8 Apr 29 3583.101 24.3 Apr 29
$18: 31: 16$ $18: 34: 25$ 21:51:16 $22: 30: 08$ $22: 57: 55$ $23: 23: 23$ $03: 51: 13$ $08: 13: 26$ 17:08:01 17:00:59 $17: 53: 28$ $18: 36: 58$ $18: 46: 59$ 22:33:48 07:17:02 08:35:18 08:38:16 $09: 43: 15$ $07: 14: 23$ 14:04:48 $14: 51: 26$ 14:58:03 $15: 10: 40$ 15:10:40 $19: 26: 37$ 18:53:54 19:21:14 20:47:41 21:01:42 21:47:11 22:01:30 $21: 43: 54$ 21:58:03 00:20:51 23:58:49 00:16:06 00:29:39

$01: 27: 20$

01:17:58 04:16:11 04:11:42 04:23:45 06:29:48 06:56:53 07:19:39 06:17:46 06:52:25 07:13:42 14:02:56 14:09:59 15:04:11 15:14:09 15:50:55 $16: 21: 52$ 16:40:07 15:57:50

$\begin{array}{ll}0.41309 & 0.02375\end{array}$ $\begin{array}{lll}0.29050 & 0.05849\end{array}$ $\begin{array}{ll}0.43242 & 0.02360\end{array}$ $0.41006 \quad 0.02423$ $0.41049 \quad 0.02452$ $\begin{array}{ll}0.26213 & 0.02403\end{array}$ $\begin{array}{lll}0.33857 & 0.05350\end{array}$ $\begin{array}{lll}0.37240 & 0.02378\end{array}$ 
3583.102 99.8 Apr 29 16:15:17 3583.103 99.8 Apr 29 16:45:45 3584.001 24.3 Apr 29 17:12:06 3584.002 99.8 Apr 29 17:45:44 3584.003 99.8 Apr 29 18:04:26 3584.101 24.3 Apr 29 17:17:41 3584.102 99.8 Apr 29 17:41:36 3584.103 99.8 Apr 29 17:59:52 3585.001 24.3 Apr 29 18:38:11 3585.002 99.8 Apr 29 19:00:34 3585.003 99.8 Apr 29 19:18:26 3585.101 24.3 Apr 29 18:44:00 3585.102 99.8 Apr 29 19:04:33 3585.103 99.8 Apr 29 19:22:42 3586.001 24.3 Apr 29 19:49:24 3586.002 99.8 Apr 29 20:13:52 3586.003 99.8 Apr 29 20:37:32 3586.004 99.8 Apr 29 20:39:56 3586.005 99.8 Apr 29 23:42:08 3586.101 24.3 Apr 29 19:53:00 3586.102 99.8 Apr 29 20:25:03 3586.103 99.8 Apr 29 20:33:53 3586.105 99.8 Apr 29 23:39:49 3587.001 24.3 Apr 30 00:53:28 3587.002 99.8 Apr 30 01:20:56 3587.003 99.8 Apr $30 \quad 01: 58: 22$ 3587.004 99.8 Apr $30 \quad 02: 31: 45$ 3587.005 99.8 Apr 30 04:19:58 3587.006 99.8 Apr 30 07:13:57 3587.007 99.8 Apr $30 \quad 09: 21: 58$ 3587.008 99.8 Apr 30 12:11:51 3587.009 99.8 Apr 30 13:10:09 3587.101 24.3 Apr 30 00:25:20 3587.102 99.8 Apr 30 01:23:11 3587.103 99.8 Apr $30 \quad 01: 35: 23$ 3587.104 99.8 Apr 30 01:59:52 3587.105 99.8 Apr $30 \quad$ 02:27:35 3587.106 99.8 Apr $30 \quad 04: 21: 46$ 3587.107 99.8 Apr 30 07:09:22 3587.108 99.8 Apr 30 09:23:19 3587.109 99.8 Apr 30 10:29:01 3587.110 99.8 Apr 30 12:04:55 3587.11199 .8 Apr 30 12:52:20 3587.112 99.8 Apr 30 13:08:44 3587.113 99.8 Apr 30 14:23:11 3588.001 24.3 Apr 30 16:10:49 3588.101 24.3 Apr 30 16:32:58 3588.102 24.3 Apr 30 17:01:50 3588.103 24.3 Apr 30 17:10:58 3589.001 24.3 Apr 30 18:48:52 3589.101 24.3 Apr 30 18:36:58 3589.102 24.3 Apr 30 18:47:28 3589.103 24.3 Apr 30 18:56:32 3590.001 24.3 Apr 30 19:46:24 3591.001 99.8 Apr 30 21:44:12 3591.002 99.8 May 1 00:04:33

\begin{tabular}{|c|c|}
\hline 0.22008 & 0.02317 \\
\hline 0.18053 & 0.02444 \\
\hline 0.47281 & 0.03050 \\
\hline 0.31225 & 0.02911 \\
\hline 0.33524 & 0.02875 \\
\hline 0.43342 & 0.02871 \\
\hline 0.24301 & 0.02996 \\
\hline 0.19990 & 0.03268 \\
\hline 0.43470 & 0.03117 \\
\hline 0.40558 & 0.03316 \\
\hline 0.35548 & 0.03315 \\
\hline 0.42958 & 0.03350 \\
\hline 0.27309 & 0.03193 \\
\hline 0.30852 & 0.03253 \\
\hline 0.46259 & 0.02559 \\
\hline 0.35579 & 0.02366 \\
\hline 0.35869 & 0.02379 \\
\hline 0.34360 & 0.02371 \\
\hline 0.33079 & 0.02374 \\
\hline 0.44329 & 0.03048 \\
\hline 0.37306 & 0.02264 \\
\hline 0.37457 & 0.02297 \\
\hline 0.38914 & 0.02313 \\
\hline 0.49352 & 0.03237 \\
\hline 0.33255 & 0.02257 \\
\hline 0.35845 & 0.02369 \\
\hline 0.30526 & 0.02359 \\
\hline 0.30189 & 0.02336 \\
\hline 0.31049 & 0.02338 \\
\hline 0.37395 & 0.02356 \\
\hline 0.34704 & 0.02350 \\
\hline 0.37378 & 0.04564 \\
\hline 0.37807 & 0.02602 \\
\hline 0.27635 & 0.02431 \\
\hline 0.23577 & 0.02409 \\
\hline 0.32539 & 0.02408 \\
\hline 0.33639 & 0.02352 \\
\hline 0.27669 & 0.02412 \\
\hline 0.29571 & 0.02290 \\
\hline 0.28660 & 0.02411 \\
\hline 0.29217 & 0.02411 \\
\hline 0.21216 & 0.02409 \\
\hline 0.23642 & 0.02409 \\
\hline 0.34207 & 0.04752 \\
\hline 0.23629 & 0.02275 \\
\hline 0.42052 & 0.02256 \\
\hline 0.44827 & 0.02268 \\
\hline 0.44051 & 0.04345 \\
\hline 0.43688 & 0.05462 \\
\hline 0.41217 & 0.02252 \\
\hline-0.31103 & 0.02250 \\
\hline-0.32968 & 0.02242 \\
\hline 0.41008 & 0.02276 \\
\hline 0.41918 & 0.02384 \\
\hline 0.34099 & 0.02348 \\
\hline 0.36703 & 0.02609 \\
\hline
\end{tabular}

$\begin{array}{ll}0.22008 & 0.02317\end{array}$ $18053 \quad 0.02444$ $\begin{array}{ll}0.47281 & 0.03050\end{array}$ $0.31225 \quad 0.02911$ $\begin{array}{ll}0.19990 & 0.03268\end{array}$ $\begin{array}{ll}0.43470 & 0.03117\end{array}$ $0.40558 \quad 0.03316$ $0.35548 \quad 0.03315$ $0.42958 \quad 0.03350$ $\begin{array}{ll}0.27309 & 0.03193\end{array}$ $\begin{array}{ll}0.30852 & 0.03253\end{array}$ $\begin{array}{ll}0.46259 & 0.02559\end{array}$ $\begin{array}{ll}0.35579 & 0.02366\end{array}$ $\begin{array}{ll}0.35869 & 0.02379\end{array}$ $\begin{array}{ll}0.34360 & 0.02371\end{array}$ $0.33079 \quad 0.02374$ $\begin{array}{ll}0.44329 & 0.03048\end{array}$ $\begin{array}{ll}0.37306 & 0.02264\end{array}$ $\begin{array}{ll}0.37457 & 0.02297\end{array}$ $\begin{array}{ll}0.38914 & 0.02313\end{array}$ $\begin{array}{ll}0.49352 & 0.03237\end{array}$ $\begin{array}{ll}0.33255 & 0.02257\end{array}$ $\begin{array}{ll}0.35845 & 0.02369\end{array}$ $\begin{array}{ll}0.30526 & 0.02359\end{array}$ 0.02336 $\begin{array}{ll}0.37395 & 0.02356\end{array}$ $\begin{array}{ll}0.34704 & 0.02350\end{array}$ $\begin{array}{lll}0.37378 & 0.04564\end{array}$ $\begin{array}{ll}0.37807 & 0.02602\end{array}$ $\begin{array}{ll}0.27635 & 0.02431\end{array}$ $\begin{array}{ll}0.23577 & 0.02409\end{array}$ $\begin{array}{lll}0.32539 & 0.02408\end{array}$ $\begin{array}{lll}0.33639 & 0.02352\end{array}$ $\begin{array}{ll}0.27669 & 0.02412\end{array}$ $\begin{array}{ll}0.29571 & 0.02290\end{array}$ $\begin{array}{ll}0.28660 & 0.02411\end{array}$ $\begin{array}{ll}0.29217 & 0.02411\end{array}$ $\begin{array}{ll}0.21216 & 0.02409\end{array}$ $0.23642 \quad 0.02409$ $\begin{array}{ll}0.34207 & 0.04752\end{array}$ $0.42052 \quad 0.02256$ $\begin{array}{ll}0.44827 & 0.02268\end{array}$ $0.44051 \quad 0.04345$ $\begin{array}{lll}0.43688 & 0.05462\end{array}$ $\begin{array}{ll}0.41217 & 0.02252\end{array}$ $\begin{array}{ll}-0.31103 & 0.02250\end{array}$ $\begin{array}{ll}-0.32968 & 0.02242\end{array}$ $\begin{array}{ll}0.41008 & 0.02276\end{array}$ $0.34099 \quad 0.02348$ $\begin{array}{ll}0.36703 & 0.02609\end{array}$
3591.10199 .8 Apr 30 3591.10299 .8 May 1 3592.001 24.3 May 1 3592.002 24.3 May 1 3592.10124 .3 May 1 3593.00199 .8 May 1 3593.10199 .8 May 1 3595.00199 .8 May 1 3595.00299 .8 May 1 3595.10199 .8 May 1 3595.10299 .8 May 1 3598.002 24.3 May 1 3598.10124 .3 May 1 3600.00124 .3 May 1 3600.002 24.3 May 1 3600.101 24.3 May 1 3600.102 24.3 May 1 3602.00124 .3 May 2 3602.00299 .8 May 2 3602.10124 .3 May 2 3602.10299 .8 May 2 3603.00124 .3 May 2 3603.00299 .8 May 2 3603.10124 .3 May 2 3603.10299 .8 May 2 3604.00124 .3 May 2 3604.00299 .8 May 2 3604.00399 .8 May 2 3604.10124 .3 May 2 3604.10299 .8 May 2 3604.10399 .8 May 2 3606.00224 .3 May 2 3606.00399 .8 May 2 3606.00499 .8 May 2 3606.10124 .3 May 2 3606.102 24.3 May 2 3606.10399 .8 May 2 3606.10499 .8 May 2 3607.00124 .3 May 2 3607.10124 .3 May 2 3607.102 24.3 May 2 3608.10124 .3 May 2 3608.102 24.3 May 2 3608.10324 .3 May 2 3608.10424 .3 May 2 3610.00124 .3 May 3 3610.00224 .3 May 3 3610.10124 .3 May 3 3610.102 24.3 May 3 3610.103 24.3 May 3 3610.10424 .3 May 3 3610.105 24.3 May 3 3610.106 24.3 May 3 3610.107 24.3 May 3 3611.00124 .3 May 3 3611.002 24.3 May 3
$21: 41: 54$ 00:02:33 03:01:37 03:15:00 $02: 55: 22$ 04:42:50 04:41:48 06:14:37 06:28:04 06:12:54 06:26:53 19:57:09 19:53:03 22:38:53

23:22:09 22:52:46 22:57:53 01:02:06 02:59:38

01:18:46 03:04:18 04:54:17 05:53:18 05:01:56 05:48:00 07:47:41 08:26:01 10:34:18 07:43:07 08:19:46 10:29:06 $16: 36: 25$ 17:20:20 20:11:20

16:14:11

$16: 34: 18$ $17: 14: 46$ 20:04:53 21:30:49 21:25:31 21:33:24 22:58:34 23:14:49 23:19:40 23:29:01 02:19:17 02:57:18 02:08:32

02:16:27

02:28:21

02:36:00

02:38:19

02:49:01

02:50:44

03:43:54

03:45:51
$0.37084 \quad 0.02240$ $\begin{array}{ll}0.37132 & 0.02419\end{array}$ $\begin{array}{ll}-0.23279 & 0.02850\end{array}$ $\begin{array}{lll}0.45969 & 0.03093\end{array}$ $\begin{array}{lll}0.39776 & 0.03138\end{array}$ $\begin{array}{ll}0.32861 & 0.02305\end{array}$ $0.27799 \quad 0.02230$ $\begin{array}{ll}0.31129 & 0.02305\end{array}$ $\begin{array}{ll}0.30703 & 0.02397\end{array}$ $\begin{array}{lll}0.34619 & 0.02357\end{array}$ $\begin{array}{ll}0.27689 & 0.02254\end{array}$ $0.44594 \quad 0.03009$ $\begin{array}{lll}0.23779 & 0.02951\end{array}$ $\begin{array}{ll}0.05227 & 0.02322\end{array}$ $\begin{array}{ll}-0.02652 & 0.02930\end{array}$ $\begin{array}{lll}0.29819 & 0.02397\end{array}$ $\begin{array}{ll}0.33521 & 0.02396\end{array}$ $\begin{array}{ll}0.33598 & 0.02230\end{array}$ $\begin{array}{ll}0.35883 & 0.02212\end{array}$ $\begin{array}{ll}0.31212 & 0.02387\end{array}$ $\begin{array}{ll}0.01723 & 0.02367\end{array}$ $\begin{array}{ll}-0.41110 & 0.02230\end{array}$ $\begin{array}{ll}0.32028 & 0.02279\end{array}$ $0.36728 \quad 0.02362$ $0.00859 \quad 0.02382$ 0.364620 .02263 $\begin{array}{ll}0.30728 & 0.02237\end{array}$ $0.30808 \quad 0.02356$ $\begin{array}{lll}-0.35137 & 0.02353\end{array}$ $\begin{array}{ll}0.27319 & 0.02359\end{array}$ $\begin{array}{lll}0.33325 & 0.02368\end{array}$ $0.37956 \quad 0.02228$ $\begin{array}{lll}0.28689 & 0.02282\end{array}$ $\begin{array}{lll}0.27769 & 0.02299\end{array}$ $\begin{array}{lll}0.33609 & 0.04453\end{array}$ $\begin{array}{ll}0.28304 & 0.02399\end{array}$ $\begin{array}{ll}0.16700 & 0.02377\end{array}$ $\begin{array}{lll}0.17416 & 0.02369\end{array}$ $\begin{array}{lll}0.39650 & 0.02221\end{array}$ $0.32500 \quad 0.02364$ $\begin{array}{lll}0.33378 & 0.02342\end{array}$ $\begin{array}{ll}0.37085 & 0.02806\end{array}$ $0.35163 \quad 0.03343$ $\begin{array}{ll}0.35661 & 0.03333\end{array}$ $\begin{array}{ll}0.39030 & 0.02370\end{array}$ $\begin{array}{ll}0.39447 & 0.02879\end{array}$ $\begin{array}{lll}0.43576 & 0.02279\end{array}$ $0.42707 \quad 0.03324$ $\begin{array}{lll}0.25389 & 0.02370\end{array}$ $\begin{array}{lll}0.42087 & 0.03333\end{array}$ $\begin{array}{ll}0.30109 & 0.02370\end{array}$ $\begin{array}{ll}0.31894 & 0.02378\end{array}$ $0.29928 \quad 0.02352$ $\begin{array}{ll}0.36565 & 0.02335\end{array}$ $\begin{array}{ll}0.39956 & 0.02362\end{array}$ $0.37688 \quad 0.02235$ 
3611.10124 .3 May 3 3612.001 24.3 May 3 3612.00299 .8 May 3 3612.00399 .8 May 3 3612.00499 .8 May 3 3612.00599 .8 May 3 3612.00699 .8 May 3 3612.101 24.3 May 3 3612.10299 .8 May 3 3612.103 99.8 May 3 3612.10499 .8 May 3 3612.10599 .8 May 3 3612.10699 .8 May 3 3613.001 24.3 May 3 3613.00299 .8 May 3 3613.00399 .8 May 3 3613.00499 .8 May 3 3613.101 24.3 May 3 3613.10299 .8 May 3 3613.103 99.8 May 3 3613.10499 .8 May 3 3613.105 99.8 May 3 3614.002 24.3 May 3 3614.00399 .8 May 3 3614.00499 .8 May 3 3614.00599 .8 May 3 3614.101 24.3 May 3 3614.10299 .8 May 3 3614.103 99.8 May 3 3614.104 99.8 May 3 3614.10599 .8 May 3 3615.001 24.3 May 3 3615.00299 .8 May 4 3615.00399 .8 May 4 3615.00499 .8 May 4 3615.00599 .8 May 4 3615.101 24.3 May 3 3615.102 24.3 May 3 3615.103 99.8 May 3 3615.104 99.8 May 4 3615.105 99.8 May 4 3615.10699 .8 May 4 3616.001 24.3 May 4 3616.101 24.3 May 4 3617.00124 .3 May 4 3617.00299 .8 May 4 3617.00399 .8 May 4 3617.00499 .8 May 4 3617.00599 .8 May 4 3617.101 24.3 May 4 3617.10299 .8 May 4 3617.103 99.8 May 4 3617.104 99.8 May 4 3617.105 99.8 May 4 3619.001 24.3 May 4 3619.10124 .3 May 4
$03: 36: 44$

$04: 35: 26$

$04: 57: 29$

$05: 13: 53$

$07: 19: 27$

09:16:40

11:50:18

04:33:16

$04: 52: 54$

05:08:56

$07: 33: 56$

$09: 22: 29$

11:55:07

$14: 48: 49$

$15: 15: 30$

$17: 27: 59$

$18: 16: 09$

14:38:38

$15: 16: 30$

$17: 32: 43$

$17: 59: 51$

18:14:11

20:16:58

$20: 36: 31$

20:55:48

$22: 52: 17$

19:54:30

$20: 31: 27$

20:51:06

22:46:49

22:59:14

$23: 36: 16$

00:05:12

$00: 31: 24$

04:08:54

04:58:23

$23: 29: 29$

$23: 31: 32$

23:58:13

$00: 23: 43$

$03: 20: 27$

$04: 55: 47$

$06: 36: 26$

$06: 41: 25$

$07: 31: 26$

08:01:36

$08: 18: 30$

08:28:17

$14: 16: 56$

$07: 22: 26$

07:59:49

$08: 17: 06$

08:27:03

14:15:21

16:38:03

16:10:30 $\begin{array}{lll}0.37347 & 0.02387\end{array}$

$0.45784 \quad 0.02280$

$0.34101 \quad 0.02355$

$0.33772 \quad 0.02296$

$\begin{array}{ll}0.34824 & 0.02271\end{array}$

$\begin{array}{ll}0.34831 & 0.02339\end{array}$

$0.27833 \quad 0.02390$

$\begin{array}{ll}0.35256 & 0.02387\end{array}$

$\begin{array}{ll}0.29162 & 0.02367\end{array}$

$0.31221 \quad 0.02359$

$0.26175 \quad 0.02367$

$0.29209 \quad 0.02358$

$\begin{array}{ll}0.25164 & 0.02376\end{array}$

$\begin{array}{ll}0.44555 & 0.02239\end{array}$

$\begin{array}{ll}0.28062 & 0.02196\end{array}$

$\begin{array}{ll}0.30734 & 0.02239\end{array}$

$\begin{array}{ll}0.33375 & 0.02349\end{array}$

$\begin{array}{ll}0.33766 & 0.02381\end{array}$

$\begin{array}{ll}0.26257 & 0.02368\end{array}$

$0.23779 \quad 0.02376$

$0.18255 \quad 0.02358$

$0.22547 \quad 0.02359$

$0.43901 \quad 0.02261$

$0.30556 \quad 0.02198$

$0.32092 \quad 0.02349$

$0.32385 \quad 0.02353$

$0.47784 \quad 0.02603$

$0.25817 \quad 0.02384$

$0.23527 \quad 0.02377$

$0.29098 \quad 0.02376$

$\begin{array}{ll}0.27519 & 0.02367\end{array}$

$\begin{array}{ll}0.39777 & 0.02362\end{array}$

$0.25419 \quad 0.02335$

$\begin{array}{ll}0.27917 & 0.02318\end{array}$

$\begin{array}{ll}0.31497 & 0.02320\end{array}$

$\begin{array}{ll}0.27541 & 0.02422\end{array}$

$0.36682 \quad 0.02384$

$0.37923 \quad 0.02388$

$\begin{array}{ll}0.20773 & 0.02388\end{array}$

$0.28487 \quad 0.02372$

$0.24155 \quad 0.02379$

$0.17749 \quad 0.02379$

$\begin{array}{ll}0.35021 & 0.02318\end{array}$

$0.39514 \quad 0.02423$

$0.41415 \quad 0.02250$

$\begin{array}{ll}0.32757 & 0.02306\end{array}$

$0.30626 \quad 0.02305$

0.284690 .02290

$0.34455 \quad 0.02377$

$\begin{array}{ll}0.45923 & 0.02374\end{array}$

$0.32057 \quad 0.02364$

$0.22593 \quad 0.02364$

$0.29876 \quad 0.02382$

$0.27301 \quad 0.02368$

$0.42150 \quad 0.02364$

$0.37019 \quad 0.02392$
3620.00124 .3 May 4 3620.00299 .8 May 4 3620.00399 .8 May 4 3620.00499 .8 May 4 3620.00599 .8 May 4 3620.10124 .3 May 4 3620.10299 .8 May 4 3620.10399 .8 May 4 3620.10499 .8 May 4 3620.10599 .8 May 4 3621.00124 .3 May 5 3621.00299 .8 May 5 3621.10124 .3 May 5 3621.10299 .8 May 5 3622.00124 .3 May 5 3622.00299 .8 May 5 3622.00399 .8 May 5 3622.00499 .8 May 5 3622.10124 .3 May 5 3622.10299 .8 May 5 3622.10399 .8 May 5 3622.10499 .8 May 5 3622.10599 .8 May 5 3624.00124 .3 May 5 3624.00224 .3 May 5 3624.00399 .8 May 5 3624.00499 .8 May 5 3624.00599 .8 May 5 3624.10124 .3 May 5 3624.10299 .8 May 5 3624.10399 .8 May 5 3624.10499 .8 May 5 3624.10599 .8 May 5 3624.10699 .8 May 5 3624.10799 .8 May 5 3624.10899 .8 May 5 3624.10999 .8 May 5 3624.11099 .8 May 5 3625.00124 .3 May 5 3625.00224 .3 May 5 3625.00324 .3 May 5 3625.00424 .3 May 5 3625.00524 .3 May 5 3625.00699 .8 May 5 3625.00799 .8 May 5 3625.00899 .8 May 5 3625.10124 .3 May 5 3625.10224 .3 May 5 3625.10324 .3 May 5 3625.10499 .8 May 5 3625.10599 .8 May 5 3625.10699 .8 May 5 3627.00124 .3 May 5 3627.00299 .8 May 5 3627.10124 .3 May 5 3627.10299 .8 May 5
18:44:05

$19: 14: 54$

19:37:18

$21: 43: 13$

23:09:47

18:36:59

19:09:52

19:32:08

21:38:07

23:03:57

$00: 46: 19$

01:09:40

00:30:18

$01: 10: 43$

02:19:08

03:03:24

$05: 15: 28$

$06: 33: 10$

02:11:09

03:10:24

$03: 51: 36$

05:20:41

$06: 30: 27$

08:08:55

08:23:01

08:40:55

09:00:27

$09: 43: 40$

07:58:04

$08: 42: 55$

$08: 57: 55$

$09: 45: 25$

09:47:29

09:49:40

09:51:23

09:53:11

09:54:30

09:56:05

$13: 29: 33$

$13: 32: 57$

$14: 18: 32$

$14: 38: 35$

$14: 49: 57$

$15: 01: 31$

$15: 26: 38$

$17: 34: 56$

$13: 38: 47$

$14: 29: 42$

$14: 30: 53$

15:00:21

$15: 24: 24$

$17: 29: 43$

$19: 30: 37$

$19: 54: 25$

$19: 25: 44$

19:40:37

$\begin{array}{ll}0.40720 & 0.02369\end{array}$

$\begin{array}{ll}0.26224 & 0.02290\end{array}$

$\begin{array}{ll}0.31946 & 0.02341\end{array}$

$\begin{array}{ll}0.26861 & 0.02359\end{array}$

$0.26907 \quad 0.02351$

$0.37304 \quad 0.02394$

$\begin{array}{ll}0.22249 & 0.02365\end{array}$

$0.27426 \quad 0.02214$ 
3627.103 99.8 May 5 3628.00124 .3 May 5 3628.10124 .3 May 5 3629.00124 .3 May 6 3629.00299 .8 May 6 3629.101 24.3 May 6 3629.10299 .8 May 6 3630.00124 .3 May 6 3630.00299 .8 May 6 3630.10124 .3 May 6 3630.102 24.3 May 6 3630.10399 .8 May 6 3634.00124 .3 May 6 3634.00299 .8 May 6 3634.00399 .8 May 6 3634.10124 .3 May 6 3634.10299 .8 May 6 3634.10399 .8 May 6 3637.00124 .3 May 6 3637.002 24.3 May 6 3637.00324 .3 May 6 3637.004 24.3 May 6 3637.005 24.3 May 6 3637.006 24.3 May 6 3637.007 24.3 May 6 3637.008 24.3 May 6 3637.009 24.3 May 6 3637.01099 .8 May 6 3637.01199 .8 May 6 3637.101 24.3 May 6 3637.102 24.3 May 6 3637.10399 .8 May 6 3637.10499 .8 May 6 3638.00124 .3 May 6 3638.10124 .3 May 6 3638.102 24.3 May 6 3638.103 24.3 May 6 3639.00124 .3 May 6 3639.002 24.3 May 6 3639.00324 .3 May 6 3639.10124 .3 May 6 3640.00124 .3 May 6 3640.00299 .8 May 6 3640.00399 .8 May 6 3640.10124 .3 May 6 3640.10299 .8 May 6 3640.10399 .8 May 6 3644.00124 .3 May 7 3644.00299 .8 May 7 3644.101 24.3 May 7 3644.10399 .8 May 7 3644.104 99.8 May 7 3646.00124 .3 May 7 3646.00299 .8 May 7 3646.00399 .8 May 7 3646.00499 .8 May 7
$19: 58: 25$

22:50:46

22:46:14

$01: 44: 52$

$02: 13: 58$

$01: 38: 23$

$02: 15: 52$

03:05:21

04:06:26

03:02:34

03:18:05

04:11:11

07:40:45

$08: 19: 14$

$08: 34: 32$

$07: 33: 38$

08:21:07

08:33:29

$11: 43: 12$

$11: 55: 42$

11:57:00

11:59:34

12:04:03

12:05:29

12:07:39

$12: 22: 14$

12:41:04

12:50:12

13:05:19

11:36:04

$12: 32: 10$

12:51:20

13:04:24

17:09:12

$16: 43: 47$

$16: 48: 36$

$16: 59: 51$

$17: 54: 41$

$17: 58: 47$

$18: 15: 42$

$17: 47: 44$

$19: 15: 18$

$19: 25: 21$

19:42:50

19:08:01

$19: 23: 40$

19:40:57

$06: 14: 15$

$06: 41: 23$

06:09:05

06:48:41

06:55:04

09:20:02

$09: 37: 12$

09:59:59

12:03:01 $\begin{array}{ll}0.16134 & 0.02734\end{array}$ $\begin{array}{ll}0.42921 & 0.02737\end{array}$ $0.32422 \quad 0.02765$ $0.39221 \quad 0.02583$ $\begin{array}{ll}0.26925 & 0.02704\end{array}$ $\begin{array}{ll}0.32876 & 0.02743\end{array}$ $0.24156 \quad 0.02724$ $\begin{array}{lll}0.45030 & 0.02744\end{array}$ $\begin{array}{ll}0.28950 & 0.02587\end{array}$ $0.41994 \quad 0.02760$ $\begin{array}{ll}0.36158 & 0.02760\end{array}$ $0.23465 \quad 0.02739$ $0.41985 \quad 0.02552$ $\begin{array}{ll}0.29427 & 0.02545\end{array}$ $\begin{array}{ll}0.32605 & 0.02563\end{array}$ $\begin{array}{ll}0.40550 & 0.02744\end{array}$ $0.28470 \quad 0.02720$ $\begin{array}{ll}0.25791 & 0.02717\end{array}$ $\begin{array}{ll}0.31103 & 0.02827\end{array}$ $0.32867 \quad 0.02558$ 0.333690 .02549 $\begin{array}{ll}0.34637 & 0.02541\end{array}$ $0.36654 \quad 0.02566$ $\begin{array}{ll}0.35891 & 0.02566\end{array}$ $0.31248 \quad 0.02549$ $\begin{array}{ll}0.30411 & 0.02541\end{array}$ $\begin{array}{ll}0.33131 & 0.02540\end{array}$ $\begin{array}{ll}0.26292 & 0.02553\end{array}$ $0.29248 \quad 0.02707$ $0.41539 \quad 0.02760$ $0.37487 \quad 0.02764$ $\begin{array}{ll}0.16234 & 0.02738\end{array}$ $0.21196 \quad 0.02703$ $\begin{array}{ll}0.36065 & 0.02566\end{array}$ $\begin{array}{ll}0.32882 & 0.02761\end{array}$ $\begin{array}{ll}0.34673 & 0.02759\end{array}$ $0.34393 \quad 0.02767$ $0.28349 \quad 0.03845$ $\begin{array}{ll}0.30821 & 0.02966\end{array}$ $\begin{array}{ll}0.35507 & 0.02210\end{array}$ $0.39822 \quad 0.02393$ $0.40170 \quad 0.02211$ $\begin{array}{ll}0.28229 & 0.02207\end{array}$ $\begin{array}{ll}0.29220 & 0.02198\end{array}$ $0.37950 \quad 0.02394$ $\begin{array}{ll}0.20704 & 0.02364\end{array}$ $\begin{array}{ll}0.23505 & 0.02381\end{array}$ $0.38962 \quad 0.02212$ $0.34710 \quad 0.02216$ $\begin{array}{ll}0.42816 & 0.02382\end{array}$ $0.03534 \quad 0.02382$ $0.08389 \quad 0.02373$ $0.39075 \quad 0.02213$ $0.30674 \quad 0.02226$ $0.28081 \quad 0.02252$ $0.27203 \quad 0.02295$
3646.00599 .8 May 7 3646.10124 .3 May 7 3646.102 24.3 May 7 3646.10399 .8 May 7 3646.10499 .8 May 7 3646.10599 .8 May 7 3646.10699 .8 May 7 3650.00124 .3 May 7 3650.00299 .8 May 7 3650.00399 .8 May 7 3650.00499 .8 May 7 3650.00599 .8 May 7 3650.00699 .8 May 7 3650.007 99.8 May 7 3650.00899 .8 May 7 3650.00999 .8 May 7 3650.01099 .8 May 7 3650.01199 .8 May 7 3650.01299 .8 May 7 3650.01399 .8 May 7 3650.10124 .3 May 7 3650.10299 .8 May 7 3650.10399 .8 May 7 3650.10499 .8 May 7 3650.10599 .8 May 7 3650.10699 .8 May 7 3654.00199 .8 May 8 3654.00299 .8 May 8 3654.10124 .3 May 8 3654.102 24.3 May 8 3654.10324 .3 May 8 3654.10499 .8 May 8 3654.10599 .8 May 8 3658.00124 .3 May 8 3658.10124 .3 May 8 3659.00124 .3 May 8 3659.00299 .8 May 8 3659.00399 .8 May 8 3659.10124 .3 May 8 3659.102 24.3 May 8 3659.10399 .8 May 8 3659.10499 .8 May 8 3669.00124 .3 May 9 3671.00124 .3 May 9 3671.00299 .8 May 9 3671.00399 .8 May 9 3671.00499 .8 May 9 3671.10124 .3 May 9 3671.105 24.3 May 9 3671.106 24.3 May 9 3671.10799 .8 May 9 3671.10899 .8 May 9 3671.10999 .8 May 9 3672.001 24.3 May 9 3672.00299 .8 May 9 3672.00399 .8 May 10

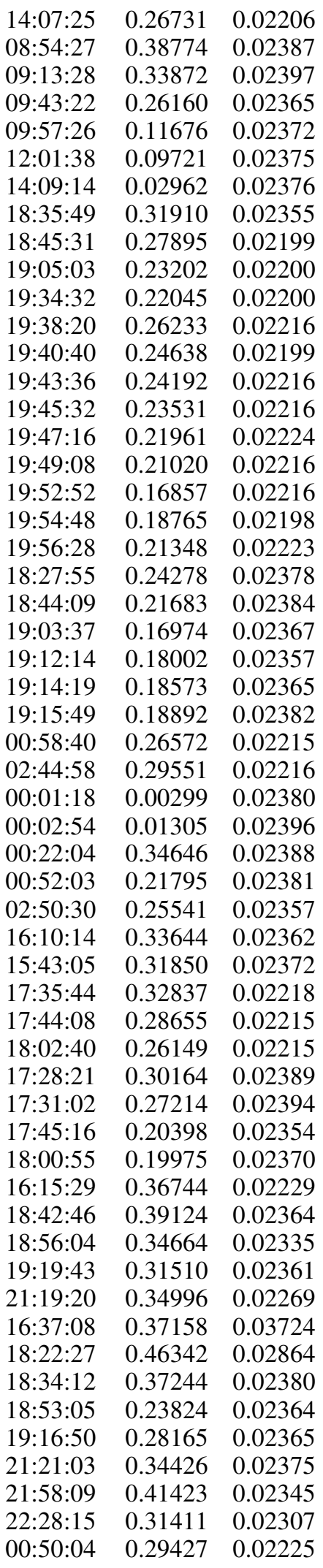


3672.00499 .8 May 10 00:51:32 3672.101 24.3 May 9 21:51:35 3672.102 99.8 May 9 22:29:48 3672.103 99.8 May 10 00:56:24 3673.001 24.3 May $10 \quad 01: 40: 18$ 3673.101 24.3 May $10 \quad 01: 32: 43$ 3674.001 24.3 May 10 02:24:41 3674.002 99.8 May $10 \quad$ 02:50:28 3674.101 24.3 May 10 02:16:43 3674.102 99.8 May 10 02:51:41 3675.001 24.3 May 10 08:36:01 3675.002 99.8 May 10 09:07:04 3675.003 99.8 May $10 \quad 09: 32: 28$ 3675.004 99.8 May 10 09:35:46 3675.00599 .8 May $10 \quad 11: 41: 39$ 3675.10124 .3 May 10 08:26:19 3675.102 24.3 May 10 08:43:33 3675.103 99.8 May 10 09:04:36 3675.104 99.8 May $10 \quad$ 09:30:38 3675.105 99.8 May $10 \quad 11: 32: 52$ 3675.106 99.8 May 10 11:58:52 3675.107 99.8 May 10 12:51:52 3675.108 99.8 May 10 12:55:05 3676.001 24.3 May 10 13:50:24 3676.00299 .8 May 10 14:01:16 3676.003 99.8 May 10 14:45:08 3676.004 99.8 May 10 16:26:10 3676.005 99.8 May $10 \quad 16: 32: 42$ 3676.101 24.3 May 10 13:38:40 3676.102 99.8 May 10 13:59:05 3676.103 99.8 May 10 14:40:56 3676.10499 .8 May $10 \quad 14: 42: 23$ 3676.105 99.8 May 10 16:12:07 3676.106 99.8 May 10 16:35:46 3676.107 99.8 May 10 16:38:37 3677.001 24.3 May 10 17:56:54 3677.002 24.3 May 10 18:21:09 3677.003 99.8 May $10 \quad 18: 33: 20$ 3677.00499 .8 May $10 \quad 18: 57: 16$ 3677.005 99.8 May 10 21:30:00 3677.101 24.3 May 10 17:43:35 3677.102 24.3 May 10 18:12:03 3677.103 99.8 May 10 18:32:06 3677.10499 .8 May 10 18:53:35 3677.105 99.8 May 10 19:03:14 3677.106 99.8 May 10 21:22:15 3677.107 99.8 May 10 21:36:45 3677.108 99.8 May 10 22:02:15 3678.001 24.3 May 11 00:05:08 3678.002 99.8 May $1100: 27: 15$ 3678.003 99.8 May $1100: 37: 25$ 3678.00499 .8 May $11 \quad 00: 38: 27$ 3678.005 99.8 May $11 \quad 00: 39: 36$ 3678.006 99.8 May $11 \quad 02: 35: 27$ 3678.101 24.3 May 10 23:57:18 3678.102 24.3 May 10 23:58:00
$0.34144 \quad 0.02368$
$\begin{array}{ll}0.44723 & 0.02789\end{array}$
0.341140 .02366
0.341140 .02366
$\begin{array}{ll}0.40169 & 0.03135\end{array}$
$\begin{array}{ll}0.33819 & 0.03373\end{array}$
$\begin{array}{ll}0.40461 & 0.03113\end{array}$
$\begin{array}{ll}0.32573 & 0.03314\end{array}$
$\begin{array}{ll}0.41342 & 0.03371\end{array}$
$\begin{array}{ll}0.36443 & 0.03333\end{array}$
$\begin{array}{ll}0.35942 & 0.03028\end{array}$
0.285090 .03286
$\begin{array}{ll}0.19837 & 0.03292\end{array}$
$\begin{array}{lll}0.29411 & 0.03283\end{array}$
$\begin{array}{ll}0.26317 & 0.02345\end{array}$
$\begin{array}{ll}-0.10426 & 0.03359\end{array}$
$\begin{array}{ll}0.34970 & 0.03380\end{array}$
$\begin{array}{ll}0.31577 & 0.03340\end{array}$
$\begin{array}{ll}0.32223 & 0.03333\end{array}$
$\begin{array}{ll}0.21087 & 0.02367\end{array}$
$\begin{array}{ll}0.23616 & 0.02368\end{array}$
$\begin{array}{ll}0.08806 & 0.02376\end{array}$
$\begin{array}{ll}0.05764 & 0.04281\end{array}$
$\begin{array}{ll}0.39052 & 0.03088\end{array}$
$\begin{array}{ll}0.27962 & 0.03269\end{array}$
$\begin{array}{ll}0.30543 & 0.02358\end{array}$
$\begin{array}{ll}0.27531 & 0.02294\end{array}$
$\begin{array}{ll}0.27265 & 0.02360\end{array}$
$\begin{array}{ll}0.40128 & 0.03375\end{array}$
$\begin{array}{ll}0.34356 & 0.03335\end{array}$
$\begin{array}{ll}0.27335 & 0.03352\end{array}$
$\begin{array}{ll}0.25282 & 0.02367\end{array}$
$\begin{array}{ll}0.14446 & 0.02377\end{array}$
$0.13297 \quad 0.02376$
0.166820 .02376
$\begin{array}{ll}0.34924 & 0.03147\end{array}$
$\begin{array}{ll}0.31878 & 0.03317\end{array}$
$\begin{array}{ll}0.27377 & 0.03068\end{array}$
$\begin{array}{ll}0.27811 & 0.02220\end{array}$
$\begin{array}{ll}0.28139 & 0.02226\end{array}$
$\begin{array}{ll}-0.04215 & 0.03377\end{array}$
$\begin{array}{ll}0.37123 & 0.03369\end{array}$
$\begin{array}{ll}0.29251 & 0.03359\end{array}$
$\begin{array}{ll}0.22803 & 0.03354\end{array}$
$\begin{array}{ll}0.27061 & 0.02367\end{array}$
$\begin{array}{ll}0.19250 & 0.02377\end{array}$
$\begin{array}{ll}0.23818 & 0.02376\end{array}$
$\begin{array}{ll}0.20872 & 0.02368\end{array}$
$\begin{array}{ll}0.30187 & 0.03120\end{array}$
$\begin{array}{ll}0.23623 & 0.03465\end{array}$
$\begin{array}{ll}0.33053 & 0.03149\end{array}$
$\begin{array}{ll}0.24524 & 0.02222\end{array}$
$\begin{array}{ll}0.28142 & 0.02230\end{array}$
$\begin{array}{ll}0.28816 & 0.02239\end{array}$
$\begin{array}{ll}0.00000 & 0.00015\end{array}$
0.374690 .02385

3678.103 99.8 May $1100: 30: 18$ 3678.104 99.8 May 11 02:36:56 3679.001 24.3 May 11 03:57:11 3679.002 99.8 May $1104: 14: 14$ 3679.003 99.8 May 11 06:52:15 3679.004 99.8 May 11 08:09:16 3679.101 24.3 May 11 03:46:35 3679.102 99.8 May 11 04:15:26 3679.103 99.8 May $1104: 21: 43$ 3679.104 99.8 May 11 06:34:37 3679.105 99.8 May 11 08:05:49 3680.001 24.3 May 11 09:37:12 3680.002 99.8 May 11 09:48:17 3680.003 99.8 May 11 10:02:05 3680.004 99.8 May 11 10:41:50 3680.005 99.8 May 11 10:48:46 3680.006 99.8 May 11 12:51:32 3680.101 24.3 May 11 09:17:43 3680.102 24.3 May 11 09:28:38 3680.103 99.8 May 11 09:46:04 3680.104 99.8 May 11 09:59:33 3680.105 99.8 May 11 12:43:29 3680.106 99.8 May 11 12:52:58 3681.002 99.8 May 11 14:45:08 3681.003 99.8 May 11 15:13:04 3681.00499 .8 May 11 15:18:42 3681.005 99.8 May 11 15:21:18 3681.006 99.8 May 11 15:34:22 3681.007 99.8 May 11 17:24:50 3681.101 24.3 May 11 14:09:00 3681.102 24.3 May 11 14:23:54 3681.103 99.8 May 11 14:43:23 3681.104 99.8 May 11 14:56:29 3681.105 99.8 May 11 15:35:51 3681.106 99.8 May 11 15:47:37 3681.107 99.8 May 11 17:21:23 3681.108 99.8 May 11 17:26:59 3682.001 24.3 May 11 18:59:07 3682.00299 .8 May 11 19:38:57 3682.101 24.3 May 11 18:51:21 3682.102 99.8 May 11 19:27:40 3682.103 99.8 May 11 19:34:58 3682.104 99.8 May 11 19:45:55 3683.001 24.3 May 11 21:52:15 3683.101 24.3 May 11 21:45:51 3684.001 24.3 May 11 23:14:34 3684.002 99.8 May 11 23:35:41 3684.003 99.8 May 11 23:45:44 3684.101 24.3 May 11 23:09:03 3684.102 99.8 May 11 23:32:35 3684.103 99.8 May 11 23:52:29 3687.001 24.3 May 12 05:27:38 3687.002 99.8 May 12 05:40:59 3687.003 99.8 May 12 05:55:15 3687.101 24.3 May 12 05:21:50 3687.102 99.8 May 12 05:36:59 $\begin{array}{ll}0.30130 & 0.02366\end{array}$ $\begin{array}{ll}0.21434 & 0.02368\end{array}$ $\begin{array}{ll}0.34166 & 0.02549\end{array}$ $\begin{array}{ll}0.28371 & 0.02317\end{array}$ $\begin{array}{ll}0.28049 & 0.02312\end{array}$ $\begin{array}{lll}0.30707 & 0.02284\end{array}$ $\begin{array}{lll}0.37806 & 0.02539\end{array}$ $\begin{array}{lll}0.23810 & 0.02366\end{array}$ $\begin{array}{lll}0.29259 & 0.02366\end{array}$ $\begin{array}{lll}0.22983 & 0.02376\end{array}$ $\begin{array}{ll}0.17151 & 0.02377\end{array}$ $\begin{array}{ll}0.37657 & 0.03137\end{array}$ $\begin{array}{ll}0.28150 & 0.02358\end{array}$ $\begin{array}{ll}0.23752 & 0.02358\end{array}$ $\begin{array}{ll}0.27125 & 0.02197\end{array}$ $\begin{array}{ll}0.26437 & 0.02357\end{array}$ $\begin{array}{ll}0.29096 & 0.02201\end{array}$ $\begin{array}{lll}-0.00474 & 0.03358\end{array}$ $\begin{array}{ll}0.37727 & 0.03369\end{array}$ $\begin{array}{ll}0.22560 & 0.02358\end{array}$ $\begin{array}{ll}0.28930 & 0.02358\end{array}$ $\begin{array}{ll}0.16680 & 0.02383\end{array}$ $\begin{array}{lll}0.13803 & 0.02382\end{array}$ $\begin{array}{ll}0.22693 & 0.03212\end{array}$ $\begin{array}{ll}0.25142 & 0.02219\end{array}$ $\begin{array}{ll}0.24904 & 0.02227\end{array}$ $\begin{array}{lll}0.28680 & 0.02203\end{array}$ $\begin{array}{ll}0.27196 & 0.02212\end{array}$ $\begin{array}{ll}0.26837 & 0.02237\end{array}$ $\begin{array}{lll}0.36231 & 0.03368\end{array}$ $\begin{array}{ll}0.34204 & 0.03376\end{array}$ $\begin{array}{ll}0.29495 & 0.03360\end{array}$ $\begin{array}{lll}0.31556 & 0.02366\end{array}$ $\begin{array}{ll}0.20682 & 0.02359\end{array}$ $\begin{array}{ll}0.27011 & 0.02366\end{array}$ $\begin{array}{ll}0.13658 & 0.02359\end{array}$ $\begin{array}{ll}0.18871 & 0.02367\end{array}$ $\begin{array}{ll}0.33894 & 0.02365\end{array}$ $\begin{array}{ll}0.26955 & 0.02255\end{array}$ $\begin{array}{lll}0.36768 & 0.03056\end{array}$ $\begin{array}{lll}0.18573 & 0.02366\end{array}$ $\begin{array}{ll}0.15797 & 0.02375\end{array}$ $\begin{array}{lll}0.19398 & 0.02375\end{array}$ $\begin{array}{lll}0.41860 & 0.02381\end{array}$ $\begin{array}{ll}0.38826 & 0.03376\end{array}$ $\begin{array}{ll}0.33067 & 0.02478\end{array}$ $\begin{array}{lll}0.26035 & 0.03152\end{array}$ $\begin{array}{ll}0.32843 & 0.02233\end{array}$ $\begin{array}{ll}0.41428 & 0.03373\end{array}$ $\begin{array}{lll}0.09482 & 0.03354\end{array}$ $\begin{array}{ll}0.08430 & 0.02369\end{array}$ $\begin{array}{ll}0.33892 & 0.02365\end{array}$ $0.28296 \quad 0.02230$ $\begin{array}{ll}0.27540 & 0.02216\end{array}$ $\begin{array}{ll}0.34637 & 0.02401\end{array}$ $\begin{array}{ll}0.30765 & 0.02367\end{array}$ 
3687.103 99.8 May $12 \quad$ 05:51:50 3688.001 24.3 May 12 06:54:27 3688.002 99.8 May 12 07:07:21 3688.003 99.8 May 12 07:23:13 3688.101 24.3 May 12 06:47:28 3688.102 99.8 May 12 07:04:07 3688.103 99.8 May 12 07:20:08 3688.104 99.8 May 12 07:25:19 3689.001 24.3 May 12 08:19:04 3689.002 24.3 May 12 08:20:25 3689.003 24.3 May 12 08:26:49 3689.004 24.3 May 12 08:28:38 3689.005 24.3 May 12 08:31:18 3689.101 24.3 May 12 08:09:29 3689.102 24.3 May 12 09:00:30 3691.00124 .3 May 12 11:08:21 3691.002 24.3 May 12 11:12:59 3691.003 24.3 May 12 11:29:31 3691.004 24.3 May 12 11:38:49 3691.005 99.8 May 12 11:59:42 3691.006 99.8 May 12 12:15:11 3691.007 99.8 May 12 13:10:37 3691.008 99.8 May $12 \quad 14: 21: 53$ 3691.101 24.3 May 12 10:50:27 3691.102 24.3 May 12 10:53:33 3691.103 24.3 May 12 11:04:02 3691.104 24.3 May 12 11:23:04 3691.105 99.8 May 12 11:56:20 3691.106 99.8 May 12 12:12:24 3691.107 99.8 May 12 13:15:02 3691.108 99.8 May 12 14:17:18 3693.001 24.3 May 12 16:35:09 3693.002 99.8 May 12 16:44:32 3693.003 99.8 May 12 17:08:37 3693.00499 .8 May 12 18:19:30 3693.005 99.8 May 12 19:24:56 3693.006 99.8 May 12 20:19:50 3693.007 99.8 May 12 21:21:08 3693.008 99.8 May 12 21:49:33 3693.101 24.3 May 12 16:27:21 3693.102 99.8 May 12 16:45:48 3693.103 99.8 May 12 17:10:06 3693.104 99.8 May 12 18:22:02 3693.105 99.8 May 12 19:19:50 3693.106 99.8 May 12 20:22:17 3693.107 99.8 May 12 21:16:59 3693.108 99.8 May 12 21:52:20 3696.001 24.3 May 13 00:36:07 3696.00299 .8 May $13 \quad 00: 54: 27$ 3696.003 99.8 May 13 01:19:33 3696.101 24.3 May 13 00:25:42 3696.102 99.8 May 13 00:51:06 3696.103 99.8 May 13 01:15:44 3698.001 24.3 May 13 11:59:13 3698.002 24.3 May 13 12:01:29 3698.003 24.3 May 13 12:16:28
$\begin{array}{lll}0.10687 & 0.02366\end{array}$ $\begin{array}{lll}0.39336 & 0.03172\end{array}$ $\begin{array}{ll}0.28954 & 0.02268\end{array}$ $\begin{array}{ll}0.30800 & 0.02280\end{array}$ $\begin{array}{ll}0.37272 & 0.03378\end{array}$ $\begin{array}{ll}0.28631 & 0.02375\end{array}$ $\begin{array}{ll}0.06948 & 0.02240\end{array}$ $\begin{array}{ll}0.04513 & 0.02374\end{array}$ $\begin{array}{ll}0.29820 & 0.03130\end{array}$ $\begin{array}{ll}0.30096 & 0.03096\end{array}$ $0.26294 \quad 0.03131$ $\begin{array}{ll}0.33238 & 0.02244\end{array}$ $\begin{array}{ll}0.29588 & 0.02252\end{array}$ $\begin{array}{ll}0.41534 & 0.03377\end{array}$ $\begin{array}{ll}0.26148 & 0.03362\end{array}$ $\begin{array}{ll}0.38315 & 0.03130\end{array}$ $\begin{array}{ll}0.39794 & 0.03154\end{array}$ $\begin{array}{ll}0.31941 & 0.03345\end{array}$ $\begin{array}{ll}0.36626 & 0.03152\end{array}$ $\begin{array}{ll}0.27250 & 0.02219\end{array}$ $\begin{array}{ll}0.30303 & 0.02226\end{array}$ $\begin{array}{ll}0.29141 & 0.02234\end{array}$ $\begin{array}{ll}0.28863 & 0.02302\end{array}$ $\begin{array}{lll}0.41786 & 0.03384\end{array}$ $\begin{array}{ll}0.38294 & 0.03375\end{array}$ $\begin{array}{ll}0.40666 & 0.03418\end{array}$ $\begin{array}{ll}0.41090 & 0.03385\end{array}$ $\begin{array}{ll}0.28317 & 0.02375\end{array}$ $\begin{array}{ll}0.25998 & 0.02375\end{array}$ $\begin{array}{ll}0.28327 & 0.02369\end{array}$ 0.249040 .02360 $\begin{array}{ll}0.38320 & 0.03330\end{array}$ $\begin{array}{ll}0.29870 & 0.03118\end{array}$ $\begin{array}{ll}0.29672 & 0.02217\end{array}$ $\begin{array}{ll}0.29103 & 0.02219\end{array}$ $\begin{array}{ll}0.29521 & 0.02210\end{array}$ $\begin{array}{ll}0.29082 & 0.02218\end{array}$ $\begin{array}{ll}0.29666 & 0.02248\end{array}$ $\begin{array}{ll}0.30181 & 0.02229\end{array}$ $\begin{array}{ll}0.42009 & 0.03374\end{array}$ $\begin{array}{ll}0.30455 & 0.03360\end{array}$ $\begin{array}{ll}0.33411 & 0.02367\end{array}$ $\begin{array}{lll}0.24567 & 0.02376\end{array}$ $\begin{array}{ll}0.26531 & 0.02377\end{array}$ $\begin{array}{ll}0.24595 & 0.02377\end{array}$ $\begin{array}{ll}0.24224 & 0.02377\end{array}$ $\begin{array}{ll}0.21982 & 0.02378\end{array}$ $\begin{array}{ll}0.34944 & 0.02218\end{array}$ $\begin{array}{ll}0.27261 & 0.02346\end{array}$ $\begin{array}{lll}0.28065 & 0.02226\end{array}$ $\begin{array}{ll}0.41389 & 0.02388\end{array}$ $\begin{array}{ll}0.28868 & 0.02375\end{array}$ $\begin{array}{ll}0.29796 & 0.02376\end{array}$ $\begin{array}{ll}0.30783 & 0.03299\end{array}$ $\begin{array}{ll}0.30142 & 0.02362\end{array}$ $0.40947 \quad 0.03297$

3698.004 99.8 May 13 12:49:03 3698.005 99.8 May 13 14:33:40 3698.101 24.3 May 13 10:29:47 3698.102 24.3 May 13 10:32:55 3698.103 24.3 May 13 10:43:53 3698.104 24.3 May 13 11:35:59 3698.105 99.8 May 13 13:11:53 3698.106 99.8 May 13 14:35:45 3698.107 99.8 May 13 14:56:41 3699.001 24.3 May 13 16:22:35 3699.002 99.8 May 13 16:31:54 3699.003 99.8 May 13 16:51:53 3699.00499 .8 May 13 18:36:43 3699.101 24.3 May 13 16:09:21 3699.102 99.8 May 13 16:33:33 3699.103 99.8 May 13 16:48:54 3699.104 99.8 May 13 18:40:55 3700.001 24.3 May 13 20:51:46 3700.002 99.8 May 13 21:02:04 3700.101 24.3 May 13 20:06:25 3700.102 24.3 May 13 20:45:17 3700.103 99.8 May 13 21:03:17 3701.001 24.3 May 13 22:03:17 3701.101 24.3 May 13 21:54:24 3702.001 24.3 May 13 23:22:39 3702.002 99.8 May 13 23:32:31 3702.003 99.8 May 13 23:51:43 3702.101 24.3 May 13 23:10:23 3702.102 99.8 May 13 23:33:50 3702.103 99.8 May 13 23:48:06 3703.001 24.3 May 14 02:04:33 3703.002 99.8 May 14 02:21:07 3703.003 99.8 May 14 02:42:44 3703.101 24.3 May 14 01:59:01 3703.102 99.8 May 14 02:34:26 3703.103 99.8 May 14 02:54:25 3704.001 24.3 May 14 04:53:34 3704.101 24.3 May 14 04:44:54 3705.001 24.3 May 14 05:42:48 3705.002 99.8 May 14 05:51:53 3705.003 99.8 May 14 06:21:13 3705.00499 .8 May 14 08:29:41 3705.005 99.8 May 14 09:43:11 3705.101 24.3 May 14 05:35:51 3705.102 24.3 May 14 05:38:07 3705.103 99.8 May 14 05:53:04 3705.104 99.8 May 14 06:12:37 3705.105 99.8 May 14 08:41:38 3705.106 99.8 May 14 09:38:33 3706.001 24.3 May 14 10:29:34 3706.002 24.3 May 14 10:31:24 3706.003 24.3 May 14 10:57:08 3706.10124 .3 May 14 10:50:38 3707.001 24.3 May 14 11:50:53 3707.101 24.3 May 14 11:42:53 3708.001 24.3 May 14 12:42:28 $\begin{array}{ll}0.29020 & 0.02358\end{array}$

$0.27907 \quad 0.02345$

$0.25880 \quad 0.03364$

$\begin{array}{ll}0.31423 & 0.02391\end{array}$

$\begin{array}{ll}0.24130 & 0.03371\end{array}$

$\begin{array}{ll}0.37985 & 0.03379\end{array}$

$0.29308 \quad 0.02376$

$\begin{array}{ll}0.17059 & 0.02379\end{array}$

$\begin{array}{lll}0.17169 & 0.02378\end{array}$

$0.33554 \quad 0.02209$

$\begin{array}{ll}0.28613 & 0.02199\end{array}$

$0.28315 \quad 0.02223$

$0.31132 \quad 0.02226$

$\begin{array}{ll}0.40043 & 0.02388\end{array}$

$\begin{array}{ll}0.27843 & 0.02367\end{array}$

$0.24983 \quad 0.02365$

$\begin{array}{ll}0.14297 & 0.02379\end{array}$

$\begin{array}{ll}0.32683 & 0.02244\end{array}$

$\begin{array}{ll}0.30065 & 0.02231\end{array}$

$0.37821 \quad 0.02373$

$\begin{array}{ll}0.40305 & 0.02384\end{array}$

$0.27654 \quad 0.02374$

$\begin{array}{ll}0.35544 & 0.02226\end{array}$

$\begin{array}{lll}0.41872 & 0.02384\end{array}$

$\begin{array}{ll}0.36512 & 0.02361\end{array}$

$\begin{array}{ll}0.30073 & 0.02281\end{array}$

$\begin{array}{ll}0.30832 & 0.02257\end{array}$

$\begin{array}{lll}0.42603 & 0.02384\end{array}$

$0.25469 \quad 0.02365$

$\begin{array}{ll}0.18278 & 0.02374\end{array}$

$\begin{array}{ll}0.39833 & 0.03237\end{array}$

$\begin{array}{lll}0.29227 & 0.02248\end{array}$

$\begin{array}{ll}0.32217 & 0.02283\end{array}$

$\begin{array}{ll}0.37637 & 0.02389\end{array}$

$\begin{array}{ll}0.15887 & 0.02357\end{array}$

$\begin{array}{ll}0.06265 & 0.02358\end{array}$

$\begin{array}{lll}0.36837 & 0.03092\end{array}$

$0.40340 \quad 0.02383$

$\begin{array}{ll}0.37142 & 0.03135\end{array}$

$0.28686 \quad 0.02214$

$0.29967 \quad 0.02351$

$0.32159 \quad 0.02351$

$0.29244 \quad 0.02226$

$0.36202 \quad 0.03356$

$0.40219 \quad 0.03382$

$0.28244 \quad 0.02373$

$\begin{array}{lll}0.25420 & 0.02374\end{array}$

$0.16560 \quad 0.02375$

$0.18299 \quad 0.02376$

$\begin{array}{ll}0.35841 & 0.02368\end{array}$

$\begin{array}{ll}0.35554 & 0.02216\end{array}$

$0.40805 \quad 0.03084$

$0.37151 \quad 0.02395$

$\begin{array}{ll}0.34175 & 0.03347\end{array}$

$\begin{array}{lll}0.41790 & 0.03369\end{array}$

$0.33429 \quad 0.03313$ 
3708.002 99.8 May 14 12:50:55 3708.003 99.8 May 14 13:18:48 3708.00499 .8 May 14 14:28:41 3708.005 99.8 May 14 15:38:14 3708.006 99.8 May 14 16:40:02 3708.007 99.8 May 14 17:11:38 3708.101 24.3 May 14 12:36:01 3708.102 99.8 May 14 12:57:04 3708.103 99.8 May 14 13:10:55 3708.104 99.8 May 14 14:34:36 3708.105 99.8 May 14 15:40:37 3708.106 99.8 May 14 16:35:56 3708.107 99.8 May 14 17:23:12 3709.00124 .3 May 14 18:10:32 3709.00299 .8 May $14 \quad 18: 27: 40$ 3709.00399 .8 May 14 18:44:51 3709.004 99.8 May 14 19:27:17 3709.10124 .3 May 14 17:55:10 3709.102 99.8 May 14 18:42:27 3709.103 99.8 May 14 19:20:48 3710.001 24.3 May 14 20:09:23 3710.002 99.8 May 14 20:19:54 3710.003 99.8 May 14 20:22:01 3710.101 24.3 May 14 19:57:30 3710.102 99.8 May 14 20:23:14 3713.001 24.3 May 14 22:07:53 3713.002 99.8 May 14 22:23:04 3713.003 99.8 May 14 22:40:52 3713.004 99.8 May $15 \quad 00: 19: 23$ 3713.005 99.8 May $15 \quad 01: 30: 26$ 3713.006 99.8 May 15 02:26:27 3713.007 99.8 May 15 03:24:10 3713.008 99.8 May $15 \quad 05: 30: 57$ 3713.10124 .3 May 14 22:02:48 3713.102 99.8 May 14 22:24:19 3713.103 99.8 May 14 22:37:04 3713.104 99.8 May 15 00:09:46 3713.105 99.8 May $15 \quad 01: 17: 04$ 3713.106 99.8 May 15 02:22:30 3713.107 99.8 May 15 03:20:32 3713.108 99.8 May $15 \quad 05: 55: 47$ 3714.001 24.3 May $15 \quad 06: 39: 12$ 3714.002 99.8 May 15 06:51:43 3714.003 99.8 May 15 07:14:20 3714.004 99.8 May $15 \quad 08: 22: 15$ 3714.005 99.8 May 15 09:27:30 3714.006 99.8 May 15 10:36:50 3714.007 99.8 May $15 \quad 11: 47: 18$ 3714.101 24.3 May 15 06:30:51 3714.102 99.8 May 15 06:47:32 3714.103 99.8 May 15 07:10:28 3714.104 99.8 May 15 08:13:23 3714.105 99.8 May 15 09:18:40 3714.106 99.8 May 15 10:44:27 3714.107 99.8 May 15 12:05:35 3720.001 24.3 May 15 19:27:56
$\begin{array}{ll}0.27650 & 0.02350\end{array}$
$\begin{array}{ll}0.28416 & 0.02267\end{array}$
$\begin{array}{ll}0.29951 & 0.02352\end{array}$
$\begin{array}{ll}0.30477 & 0.02370\end{array}$
$\begin{array}{ll}0.31064 & 0.02354\end{array}$
$\begin{array}{ll}0.30831 & 0.02286\end{array}$
$\begin{array}{ll}0.47589 & 0.03364\end{array}$
$\begin{array}{ll}0.26087 & 0.02375\end{array}$
$\begin{array}{ll}0.31302 & 0.02375\end{array}$
$\begin{array}{ll}0.25576 & 0.02376\end{array}$
$\begin{array}{ll}0.23866 & 0.02376\end{array}$
$\begin{array}{ll}0.20467 & 0.02376\end{array}$
$\begin{array}{lll}0.07469 & 0.02259\end{array}$
$\begin{array}{lll}0.33778 & 0.02250\end{array}$
$\begin{array}{ll}0.30580 & 0.02339\end{array}$
$\begin{array}{ll}0.32644 & 0.02345\end{array}$
$\begin{array}{ll}0.33847 & 0.02348\end{array}$
$\begin{array}{ll}0.34513 & 0.02385\end{array}$
$\begin{array}{ll}0.20817 & 0.02375\end{array}$
$\begin{array}{ll}0.11414 & 0.02377\end{array}$
$\begin{array}{ll}-0.03005 & 0.02325\end{array}$
$\begin{array}{ll}0.00000 & 0.02346\end{array}$
$\begin{array}{ll}-0.03201 & 0.02204\end{array}$
$\begin{array}{ll}0.39293 & 0.02386\end{array}$
$\begin{array}{ll}0.25386 & 0.02365\end{array}$
$\begin{array}{ll}0.38982 & 0.02208\end{array}$
$\begin{array}{ll}0.30484 & 0.02188\end{array}$
$\begin{array}{ll}0.32439 & 0.02206\end{array}$
$\begin{array}{ll}0.32925 & 0.02190\end{array}$
$\begin{array}{ll}0.28663 & 0.02183\end{array}$
$\begin{array}{ll}0.31359 & 0.02351\end{array}$
$\begin{array}{ll}0.29255 & 0.02201\end{array}$
$\begin{array}{lll}0.33649 & 0.02344\end{array}$
$\begin{array}{ll}0.38788 & 0.02404\end{array}$
$\begin{array}{lll}0.24356 & 0.02356\end{array}$
$\begin{array}{ll}0.32807 & 0.02356\end{array}$
$\begin{array}{ll}0.30294 & 0.02367\end{array}$
$\begin{array}{ll}0.29351 & 0.02383\end{array}$
$\begin{array}{ll}0.32121 & 0.02376\end{array}$
$\begin{array}{ll}0.30870 & 0.02367\end{array}$
$\begin{array}{ll}0.22842 & 0.02376\end{array}$
$\begin{array}{lll}0.41099 & 0.03089\end{array}$
$\begin{array}{ll}0.28341 & 0.02347\end{array}$
$\begin{array}{ll}0.33286 & 0.02208\end{array}$
$\begin{array}{ll}0.27017 & 0.02356\end{array}$
$\begin{array}{ll}0.28516 & 0.02190\end{array}$
$\begin{array}{ll}0.34440 & 0.02342\end{array}$
$\begin{array}{ll}0.32201 & 0.02211\end{array}$
$0.42654 \quad 0.02292$
$\begin{array}{ll}0.20877 & 0.02365\end{array}$
$\begin{array}{ll}0.23986 & 0.02373\end{array}$
$\begin{array}{ll}0.25298 & 0.02367\end{array}$
0.299130 .02376
$\begin{array}{ll}0.29705 & 0.02376\end{array}$
$\begin{array}{ll}0.25339 & 0.02375\end{array}$
$0.42079 \quad 0.03124$

3720.00299 .8 May $15 \quad 19: 41: 27$ 3720.003 99.8 May 15 20:33:24 3720.004 99.8 May 15 22:02:47 3720.005 99.8 May 15 23:15:04 3720.006 99.8 May 16 00:04:27 3720.103 24.3 May 15 19:25:05 3720.104 99.8 May 15 19:38:20 3720.105 99.8 May 15 19:57:30 3720.106 99.8 May 15 20:21:14 3720.107 99.8 May 15 20:31:14 3720.108 99.8 May 15 22:04:20 3720.109 99.8 May 15 23:11:14 3720.110 99.8 May 16 00:08:06 3721.00124 .3 May $16 \quad 01: 32: 26$ 3721.002 99.8 May 16 01:56:15 3721.00499 .8 May 16 02:20:40 3721.005 99.8 May 16 02:43:08 3721.006 99.8 May 16 03:45:13 3721.007 99.8 May 16 04:57:31 3721.008 99.8 May 16 06:27:39 3721.101 24.3 May 16 01:20:10 3721.102 99.8 May $16 \quad 01: 58: 18$ 3721.103 99.8 May 16 02:10:43 3721.10499 .8 May 16 02:40:15 3721.105 99.8 May 16 03:47:35 3721.106 99.8 May 16 04:54:20 3721.107 99.8 May 16 06:18:19 3724.001 24.3 May 16 18:48:39 3724.103 24.3 May 16 18:16:18 3724.104 24.3 May 16 18:39:03 3725.001 24.3 May 16 19:41:01 3725.002 99.8 May 16 19:54:02 3725.101 24.3 May 16 19:30:36 3725.102 24.3 May 16 19:34:59 3725.103 99.8 May 16 19:49:54 3731.001 24.3 May 17 04:47:24 3731.003 99.8 May 17 05:08:59 3731.004 99.8 May 17 05:13:29 3731.005 99.8 May 17 05:27:17 3731.101 24.3 May 17 04:38:06 3731.102 99.8 May 17 04:55:04 3731.103 99.8 May 17 05:29:21 3731.104 99.8 May 17 05:42:51 3731.105 99.8 May 17 05:45:31 3732.001 24.3 May 17 09:08:45 3732.002 99.8 May 17 09:20:10 3732.003 99.8 May 17 09:45:19 3732.00499 .8 May 17 11:20:18 3732.005 99.8 May 17 11:27:16 3732.006 99.8 May 17 12:37:40 3732.007 99.8 May 17 14:27:00 3732.101 24.3 May 17 09:00:27 3732.102 99.8 May 17 09:16:46 3732.103 99.8 May 17 09:23:03 3732.104 99.8 May 17 09:43:03 3732.105 99.8 May 17 11:17:59 $\begin{array}{ll}0.28666 & 0.03296\end{array}$

$\begin{array}{ll}0.27603 & 0.03327\end{array}$

$\begin{array}{ll}0.27837 & 0.02214\end{array}$

$\begin{array}{ll}0.29194 & 0.02190\end{array}$

$\begin{array}{ll}0.31104 & 0.02282\end{array}$

$0.36264 \quad 0.03760$

$\begin{array}{ll}0.22356 & 0.03720\end{array}$

$\begin{array}{ll}0.13434 & 0.05510\end{array}$

$\begin{array}{ll}0.16442 & 0.05920\end{array}$

$\begin{array}{lll}0.22523 & 0.03709\end{array}$

$\begin{array}{ll}0.26200 & 0.02644\end{array}$

$\begin{array}{lll}0.19973 & 0.02636\end{array}$

$\begin{array}{ll}0.26124 & 0.02627\end{array}$

$\begin{array}{ll}0.20414 & 0.03018\end{array}$

$\begin{array}{ll}0.27128 & 0.02265\end{array}$

$\begin{array}{ll}0.31233 & 0.02352\end{array}$

$\begin{array}{ll}0.33146 & 0.02237\end{array}$

$\begin{array}{ll}0.26348 & 0.02213\end{array}$

$\begin{array}{ll}0.33314 & 0.02350\end{array}$

$\begin{array}{ll}0.25657 & 0.02342\end{array}$

$\begin{array}{ll}0.35792 & 0.02618\end{array}$

$\begin{array}{lll}0.22168 & 0.02644\end{array}$

$\begin{array}{lll}0.25257 & 0.02631\end{array}$

$\begin{array}{lll}0.31114 & 0.02641\end{array}$

$\begin{array}{ll}0.23070 & 0.02643\end{array}$

$\begin{array}{ll}0.28413 & 0.02653\end{array}$

$\begin{array}{lll}0.28673 & 0.02652\end{array}$

$\begin{array}{ll}0.27054 & 0.03349\end{array}$

$\begin{array}{lll}0.38533 & 0.02614\end{array}$

$\begin{array}{ll}0.38880 & 0.03681\end{array}$

$\begin{array}{ll}0.37806 & 0.03122\end{array}$

$\begin{array}{lll}0.37806 & 0.03122\end{array}$

$\begin{array}{lll}0.25571 & 0.03686\end{array}$

$\begin{array}{lll}0.27230 & 0.03695\end{array}$

$\begin{array}{ll}0.20477 & 0.02627\end{array}$

$\begin{array}{lll}0.39064 & 0.03090\end{array}$

$\begin{array}{ll}0.34059 & 0.02224\end{array}$

$\begin{array}{lll}0.32523 & 0.02206\end{array}$

$\begin{array}{lll}0.29437 & 0.02195\end{array}$

$\begin{array}{lll}0.43768 & 0.03700\end{array}$

$\begin{array}{ll}0.30230 & 0.02597\end{array}$

$\begin{array}{ll}0.28516 & 0.02639\end{array}$

$\begin{array}{lll}0.28813 & 0.02625\end{array}$

$\begin{array}{lll}0.27300 & 0.02608\end{array}$

$\begin{array}{lll}0.39927 & 0.02234\end{array}$

$\begin{array}{lll}0.33906 & 0.02366\end{array}$

$\begin{array}{lll}0.25868 & 0.02354\end{array}$

$\begin{array}{lll}0.16907 & 0.02274\end{array}$

$\begin{array}{ll}0.24168 & 0.02273\end{array}$

$\begin{array}{ll}0.22801 & 0.02233\end{array}$

$\begin{array}{ll}0.20400 & 0.02336\end{array}$

$\begin{array}{lll}0.36958 & 0.02623\end{array}$

$\begin{array}{ll}0.16665 & 0.02594\end{array}$

$\begin{array}{ll}0.28333 & 0.02611\end{array}$

$\begin{array}{lll}0.28319 & 0.02581\end{array}$

$0.23840 \quad 0.02600$ 
3732.106 99.8 May 17 12:42:27 3732.107 99.8 May 17 14:24:35 3733.001 24.3 May 17 15:58:55 3733.002 99.8 May 17 16:15:55 3733.004 99.8 May 17 16:57:51 3733.005 99.8 May 17 19:57:00 3733.006 99.8 May 17 21:10:40 3733.101 24.3 May 17 15:48:06 3733.102 99.8 May 17 16:13:44 3733.103 99.8 May 17 16:20:23 3733.104 99.8 May 17 16:48:52 3733.105 99.8 May 17 19:58:52 3733.106 99.8 May 17 21:06:57 3734.00124 .3 May 17 23:15:51 3734.002 99.8 May 17 23:24:09 3734.003 99.8 May 17 23:51:12 3734.101 24.3 May 17 23:05:24 3734.102 99.8 May 17 23:30:47 3734.103 99.8 May 17 23:44:50 3735.001 24.3 May 18 01:21:07 3735.002 24.3 May $18 \quad 02: 21: 15$ 3735.003 99.8 May 18 02:37:37 3735.004 99.8 May 18 03:07:12 3735.101 24.3 May 18 02:12:46 3735.102 24.3 May 18 02:15:35 3735.103 99.8 May 18 02:29:32 3735.104 99.8 May 18 02:59:09 3735.105 99.8 May $18 \quad 04: 19: 23$ 3737.001 24.3 May 18 05:07:54 3737.002 24.3 May 18 10:14:20 3737.101 24.3 May 18 05:02:30 3737.102 24.3 May 18 05:05:23 3737.103 24.3 May 18 05:15:55 3737.104 24.3 May 18 10:15:30 3739.00124 .3 May 18 14:09:48 3739.002 24.3 May 18 14:11:17 3739.101 24.3 May 18 13:43:58 3739.102 24.3 May 18 13:57:29 3740.00124 .3 May 18 14:58:17 3740.002 99.8 May 18 15:08:15 3740.10124 .3 May 18 14:47:51 3740.102 99.8 May 18 15:06:31 3742.001 99.8 May 18 17:21:19 3742.101 24.3 May 18 16:37:51 3742.102 24.3 May 18 16:50:49 3742.103 99.8 May 18 17:20:08 3742.10499 .8 May 18 17:42:37 3746.00199 .8 May 19 20:14:14 3747.001 24.3 May 19 22:55:19 3747.002 99.8 May 19 23:08:40 3747.101 24.3 May 19 22:21:13 3747.102 24.3 May 19 22:46:29 3747.103 99.8 May 19 23:06:05 3748.001 99.8 May 20 00:59:41 3748.101 24.3 May 20 00:32:40 3748.10299 .8 May 20 01:01:17

\begin{tabular}{|c|c|}
\hline 0.22480 & 0.02599 \\
\hline 0.26236 & 0.02602 \\
\hline 0.42447 & 0.02191 \\
\hline 0.29596 & 0.02231 \\
\hline 0.32939 & 0.02203 \\
\hline 0.32618 & 0.02200 \\
\hline 0.27260 & 0.02193 \\
\hline 0.39628 & 0.02620 \\
\hline 0.19626 & 0.02623 \\
\hline 0.22751 & 0.02608 \\
\hline 0.26397 & 0.02603 \\
\hline 0.23118 & 0.02637 \\
\hline 0.19146 & 0.02638 \\
\hline 0.40421 & 0.03298 \\
\hline 0.30299 & 0.02189 \\
\hline 0.32104 & 0.02195 \\
\hline 0.38648 & 0.02599 \\
\hline 0.23682 & 0.02604 \\
\hline 0.21704 & 0.02611 \\
\hline 0.41174 & 0.03332 \\
\hline 0.38299 & 0.03333 \\
\hline 0.31697 & 0.02216 \\
\hline 0.30289 & 0.02196 \\
\hline 0.33177 & 0.03694 \\
\hline 0.35556 & 0.03707 \\
\hline 0.17922 & 0.02589 \\
\hline 0.23861 & 0.02597 \\
\hline 0.22367 & 0.02587 \\
\hline-0.08781 & 0.03291 \\
\hline 0.00646 & 0.03106 \\
\hline-0.06269 & 0.03673 \\
\hline 0.06153 & \\
\hline-0.04502 & 0.03740 \\
\hline-0.04555 & 0.03727 \\
\hline 0.49672 & 0.03098 \\
\hline 0.44851 & 0.02191 \\
\hline 0.48952 & 0.03677 \\
\hline 0.41182 & 0.03697 \\
\hline 0.45227 & 0.02350 \\
\hline 0.33927 & 0.02407 \\
\hline 0.38932 & 0.03703 \\
\hline 0.24091 & 0.03688 \\
\hline 0.39097 & 0.03130 \\
\hline 0.42612 & 0.03676 \\
\hline 0.39437 & 0.03691 \\
\hline 0.19486 & 0.03716 \\
\hline 0.28605 & 0.03697 \\
\hline 0.41596 & 0.03769 \\
\hline 0.37611 & 0.03679 \\
\hline 0.28526 & 0.03638 \\
\hline 0.42867 & 0.03696 \\
\hline 0.40001 & 0.03646 \\
\hline 0.29252 & 0.03668 \\
\hline 0.35331 & 0.03638 \\
\hline 0.43307 & 0.03641 \\
\hline 0.26406 & 0.03634 \\
\hline
\end{tabular}

$\begin{array}{lll}0.22480 & 0.02599\end{array}$ $0.26236 \quad 0.02602$ 0.42447 $0.29596 \quad 0.02231$ $\begin{array}{lll}0.32939 & 0.02203\end{array}$ $\begin{array}{ll}0.32618 & 0.02200\end{array}$ $0.27260 \quad 0.02193$ 0.02620 $\begin{array}{ll}0.26397 & 0.02603\end{array}$ $0.23118 \quad 0.02637$ $\begin{array}{ll}0.19146 & 0.02638\end{array}$ $\begin{array}{ll}0.40421 & 0.03298\end{array}$ $\begin{array}{ll}0.30299 & 0.02189\end{array}$ $\begin{array}{ll}0.38648 & 0.02599\end{array}$ $\begin{array}{ll}0.23682 & 0.02604\end{array}$ $\begin{array}{ll}0.21704 & 0.02611 \\ 0.41174 & 0.03332\end{array}$ $\begin{array}{lll}0.38299 & 0.03333\end{array}$ $0.31697 \quad 0.02216$ $0.30289 \quad 0.02196$ $\begin{array}{lll}0.33177 & 0.03694\end{array}$ $\begin{array}{ll}0.23861 & 0.02597\end{array}$ $\begin{array}{lll}0.22367 & 0.02587\end{array}$ $\begin{array}{ll}-0.08781 & 0.03291\end{array}$ $\begin{array}{lll}0.00646 & 0.03106\end{array}$ 0.03673 $\begin{array}{ll}0.06153 & 0.03719\end{array}$ $-0.04502 \quad 0.03740$ $\begin{array}{ll}-0.04555 & 0.03727\end{array}$ 0.0306720 .08 $\begin{array}{ll}0.44851 & 0.02191\end{array}$ $\begin{array}{ll}0.41182 & 0.03697\end{array}$ $0.45227 \quad 0.02350$ $\begin{array}{ll}0.33927 & 0.02407\end{array}$ $0.24091 \quad 0.03688$ $0.39097 \quad 0.03130$ $0.39437-0.03691$ $0.19486 \quad 0.03716$ $0.28605 \quad 0.03697$ $\begin{array}{lll}0.37611 & 0.03679\end{array}$ $0.28526 \quad 0.03638$ $\begin{array}{lll}0.42867 & 0.03696\end{array}$ $0.40001 \quad 0.03646$ $\begin{array}{ll}0.35331 & 0.03638\end{array}$ $\begin{array}{ll}0.43307 & 0.03641 \\ 0.26406 & 0.03634\end{array}$ $0.26406 \quad 0.03634$
3749.00124 .3 May $20 \quad 02: 44: 48$ 3749.002 99.8 May 20 02:49:29 3749.101 24.3 May 20 02:27:58 3749.10299 .8 May 20 02:51:31 3750.00199 .8 May $20 \quad 04: 51: 58$ 3750.101 24.3 May 20 04:36:59 3750.10299 .8 May 20 04:53:18 3751.001 24.3 May 20 14:40:19 3751.002 99.8 May 20 14:49:20 3751.00299 .8 May 20 14:49:20 3751.101 24.3 May 20 14:29:47 3751.102 99.8 May 20 14:47:29 3752.001 99.8 May 20 19:56:29 3755.00124 .3 May 21 06:13:11 3756.001 99.8 May 21 07:36:12 3756.002 99.8 May 21 20:04:22 3756.003 99.8 May 21 20:32:13 3756.10199 .8 May 21 07:30:36 3756.102 99.8 May 21 20:07:42 3756.103 99.8 May 21 20:22:32 3757.00124 .3 May $2200: 15: 33$ 3757.002 99.8 May $2200: 24: 52$ 3757.00399 .8 May 22 01:04:23 3757.00499 .8 May 22 02:20:36 3757.005 99.8 May $22 \quad 03: 46: 35$ 3757.006 99.8 May 22 04:37:48 3757.101 99.8 May 22 00:47:16 3757.10299 .8 May 22 01:01:18 3757.103 99.8 May 22 02:22:58 3757.10499 .8 May 22 03:40:42 3757.105 99.8 May 22 04:40:56 3759.001 24.3 May 22 06:23:20 3759.002 24.3 May 22 07:31:05 3759.003 99.8 May 22 07:48:26 3759.004 99.8 May 22 09:28:57 3759.005 99.8 May 22 12:34:09 3759.006 99.8 May 22 14:16:06 3759.007 99.8 May 22 14:35:12 3759.101 24.3 May 22 06:15:53 3759.10299 .8 May 22 07:43:56 3759.103 99.8 May 22 09:35:42 3759.10499 .8 May 22 12:25:34 3759.10599 .8 May $22 \quad 14: 17: 45$ 3762.001 24.3 May 22 20:44:27 3763.00124 .3 May 22 22:40:42 3764.001 24.3 May 23 01:06:58 3764.00299 .8 May $2301: 31: 38$ 3764.003 99.8 May 23 01:36:12 3764.004 99.8 May 23 03:04:25 3764.005 99.8 May 23 04:12:52 3764.006 99.8 May 23 05:44:15 3764.10199 .8 May $2301: 44: 25$ 3764.10299 .8 May 23 03:12:58 3764.103 99.8 May 23 04:22:03 3764.104 99.8 May 23 05:51:39 3765.00124 .3 May 23 07:29:18
$0.32981 \quad 0.03691$ $\begin{array}{ll}0.34581 & 0.03713\end{array}$ $0.42656 \quad 0.03644$ $0.32488 \quad 0.03646$ $\begin{array}{ll}0.31988 & 0.03687\end{array}$ $\begin{array}{ll}0.44352 & 0.03674\end{array}$ $0.36990 \quad 0.03620$ $\begin{array}{ll}0.39605 & 0.03699\end{array}$ $\begin{array}{ll}0.37497 & 0.02682\end{array}$ $\begin{array}{ll}0.37497 & 0.02682\end{array}$ $\begin{array}{ll}0.36047 & 0.03670\end{array}$ $0.29108 \quad 0.03634$ $0.36549 \quad 0.02654$ $\begin{array}{ll}0.38612 & 0.02641\end{array}$ $0.37228 \quad 0.03736$ $0.34034 \quad 0.03738$ $0.36698 \quad 0.02657$ $\begin{array}{lll}0.36498 & 0.02229\end{array}$ $0.30996 \quad 0.03553$ $\begin{array}{lll}0.30933 & 0.02829\end{array}$ $\begin{array}{ll}0.36717 & 0.03695\end{array}$ $0.30298 \quad 0.02617$ $\begin{array}{lll}0.29509 & 0.02627\end{array}$ $\begin{array}{ll}0.32220 & 0.02635\end{array}$ $\begin{array}{lll}0.29725 & 0.02637\end{array}$ $\begin{array}{ll}0.26408 & 0.02653\end{array}$ $\begin{array}{lll}0.34147 & 0.02363\end{array}$ $\begin{array}{lll}0.32739 & 0.02342\end{array}$ $\begin{array}{lll}0.36104 & 0.02388\end{array}$ $0.31512 \quad 0.02380$ $\begin{array}{ll}0.29581 & 0.02388\end{array}$ $\begin{array}{lll}0.34559 & 0.02627\end{array}$ $\begin{array}{ll}0.31392 & 0.02627\end{array}$ $\begin{array}{ll}0.29162 & 0.02617\end{array}$ $0.29572 \quad 0.02644$ $0.32897 \quad 0.02628$ $\begin{array}{lll}0.35267 & 0.02671\end{array}$ $\begin{array}{lll}0.30728 & 0.02672\end{array}$ $\begin{array}{ll}0.37415 & 0.02247\end{array}$ $0.32608 \quad 0.02376$ $\begin{array}{lll}0.34963 & 0.02388\end{array}$ $\begin{array}{ll}0.39949 & 0.02379\end{array}$ $\begin{array}{ll}0.38792 & 0.02802\end{array}$ $\begin{array}{ll}0.34091 & 0.03703\end{array}$ $0.36998 \quad 0.03685$ $\begin{array}{lll}0.35329 & 0.03718\end{array}$ $\begin{array}{lll}0.35729 & 0.03742\end{array}$ $\begin{array}{ll}0.27144 & 0.02632\end{array}$ $0.34072 \quad 0.02633$ $0.28777 \quad 0.02642$ $\begin{array}{lll}0.28665 & 0.02635\end{array}$ $\begin{array}{ll}0.36355 & 0.02439\end{array}$ $0.32076 \quad 0.02447$ $\begin{array}{ll}0.33652 & 0.02558\end{array}$ $\begin{array}{ll}0.37200 & 0.02667\end{array}$ $0.38523 \quad 0.02625$ 
3765.002 99.8 May 23 08:33:43 3765.003 99.8 May 23 10:09:03 3765.10199 .8 May 23 08:57:04 3765.102 99.8 May 23 09:59:46 3765.103 99.8 May 23 11:08:47 3767.001 24.3 May 23 14:04:38 3767.00299 .8 May $2314: 14: 53$ 3767.003 99.8 May 23 15:04:10 3767.10199 .8 May 23 14:12:29 3767.102 99.8 May 23 14:40:17 3769.001 24.3 May 24 01:12:25 3769.00299 .8 May $2401: 30: 42$ 3769.003 99.8 May 24 01:47:06 3769.00499 .8 May 24 03:25:22 3769.00599 .8 May 24 05:49:35 3769.006 99.8 May 24 08:19:35 3769.101 99.8 May 24 01:32:07 3769.10299 .8 May 24 01:44:26 3769.103 99.8 May 24 03:26:39 3769.104 99.8 May 24 05:45:51 3769.105 99.8 May 24 08:15:19 3770.001 24.3 May 24 09:35:00 3770.002 24.3 May 24 10:25:41 3770.00399 .8 May 24 10:41:45 3770.102 99.8 May 24 12:09:57 3774.00124 .3 May 25 02:44:57 3774.002 99.8 May 25 03:15:21 3774.003 99.8 May $25 \quad 03: 24: 52$ 3774.101 99.8 May $25 \quad 03: 19: 42$ 3776.001 24.3 May 25 09:34:35 3778.00199 .8 May 25 13:51:06 3778.00299 .8 May 25 14:14:04 3778.101 99.8 May 25 14:00:10 3778.102 99.8 May $25 \quad 14: 16: 52$ 3779.001 24.3 May 25 16:40:05 3779.00299 .8 May $25 \quad 16: 51: 43$ 3779.003 99.8 May 25 16:55:25 3779.004 99.8 May 25 17:09:16 3779.00599 .8 May 25 17:38:11 3779.006 99.8 May 25 18:00:19 3779.101 99.8 May 25 16:50:19 3779.10299 .8 May 25 17:07:41 3779.103 99.8 May 25 18:03:39 3780.001 24.3 May 25 19:31:06 3780.002 99.8 May 25 19:57:44 3780.003 99.8 May 25 20:01:32 3780.00499 .8 May 25 21:02:10 3780.00599 .8 May 25 21:05:13 3780.006 99.8 May 25 21:59:15 3780.007 99.8 May 25 22:02:22 3780.008 99.8 May 25 23:05:24 3780.009 99.8 May 25 23:08:43 3780.010 99.8 May $26 \quad 00: 29: 20$ 3780.01199 .8 May $2600: 32: 53$ 3780.01299 .8 May $26 \quad 01: 35: 43$ 3780.01399 .8 May 26 01:46:07
0.33304
0.27023
0.02632
0.33622
0.02643
$\begin{array}{lll}0.31296 & 0.02429\end{array}$
$\begin{array}{ll}0.17923 & 0.02439\end{array}$
$\begin{array}{ll}0.38075 & 0.02634\end{array}$
$\begin{array}{ll}0.24809 & 0.02615\end{array}$
$\begin{array}{ll}0.22497 & 0.02634\end{array}$
$\begin{array}{ll}0.32663 & 0.02370\end{array}$
$\begin{array}{lll}0.28087 & 0.02457\end{array}$
$\begin{array}{ll}0.31322 & 0.02639\end{array}$
$0.28642 \quad 0.02614$
$\begin{array}{ll}0.26501 & 0.02649\end{array}$
$\begin{array}{lll}0.24737 & 0.02634\end{array}$
$\begin{array}{ll}0.25080 & 0.02612\end{array}$
$\begin{array}{ll}0.27505 & 0.02655\end{array}$
$\begin{array}{ll}0.33762 & 0.02354\end{array}$
$\begin{array}{lll}0.34637 & 0.02439\end{array}$
$\begin{array}{ll}0.35277 & 0.02441\end{array}$
$\begin{array}{ll}0.39188 & 0.02424\end{array}$
$\begin{array}{ll}0.32872 & 0.02426\end{array}$
$\begin{array}{ll}0.31487 & 0.02651\end{array}$
$\begin{array}{ll}0.34973 & 0.03723\end{array}$
$\begin{array}{ll}0.26174 & 0.02622\end{array}$
0.304420 .02446
$0.32527 \quad 0.02626$
$\begin{array}{ll}0.24924 & 0.02633\end{array}$
$\begin{array}{ll}0.22566 & 0.02625\end{array}$
$\begin{array}{ll}0.36931 & 0.02420\end{array}$
$\begin{array}{ll}0.32442 & 0.03743\end{array}$
$\begin{array}{ll}0.08417 & 0.03658\end{array}$
$\begin{array}{ll}0.07757 & 0.02611\end{array}$
$\begin{array}{ll}0.27708 & 0.02472\end{array}$
$\begin{array}{ll}0.25065 & 0.02474\end{array}$
$\begin{array}{ll}0.32666 & 0.02622\end{array}$
$\begin{array}{lll}0.05934 & 0.02634\end{array}$
$\begin{array}{ll}0.04982 & 0.02604\end{array}$
$0.04854 \quad 0.02616$
$\begin{array}{ll}0.05608 & 0.02648\end{array}$
$\begin{array}{ll}0.05107 & 0.03359\end{array}$
0.293610 .02506
$\begin{array}{ll}0.23115 & 0.02416\end{array}$
$\begin{array}{ll}0.20987 & 0.02247\end{array}$
$\begin{array}{ll}0.41072 & 0.02708\end{array}$
$\begin{array}{ll}0.30448 & 0.02612\end{array}$
$\begin{array}{ll}0.28904 & 0.02617\end{array}$
$\begin{array}{ll}0.31155 & 0.02633\end{array}$
0.237290 .02616
$\begin{array}{ll}0.28732 & 0.02632\end{array}$
$\begin{array}{ll}0.26756 & 0.02641\end{array}$
$\begin{array}{ll}0.25893 & 0.02642\end{array}$
$\begin{array}{ll}0.22689 & 0.02635\end{array}$
$\begin{array}{ll}0.25444 & 0.02629\end{array}$
$\begin{array}{ll}0.25226 & 0.02627\end{array}$
$\begin{array}{ll}0.24627 & 0.02618\end{array}$
$0.23762 \quad 0.02643$

3780.014 99.8 May 26 03:22:08 3780.015 99.8 May $26 \quad 03: 27: 19$ 3780.101 24.3 May 25 19:22:21 3780.102 99.8 May 25 19:59:17 3780.103 99.8 May 25 20:08:54 3780.104 99.8 May 25 21:03:43 3780.105 99.8 May 25 21:13:03 3780.106 99.8 May 25 22:00:57 3780.107 99.8 May 25 22:10:25 3780.108 99.8 May 25 22:12:11 3780.109 99.8 May 25 23:07:01 3780.110 99.8 May 25 23:16:01 3780.11199 .8 May 26 00:12:29 3780.11299 .8 May 26 00:14:55 3780.113 99.8 May 26 01:40:08 3780.11499 .8 May $26 \quad 01: 54: 49$ 3780.115 99.8 May $26 \quad 03: 23: 58$ 3780.116 99.8 May 26 03:36:11 3781.001 24.3 May 26 06:27:15 3784.001 24.3 May 26 09:54:35 3784.002 99.8 May 26 10:06:16 3784.003 99.8 May 26 10:15:30 3784.004 99.8 May 26 10:33:26 3784.101 99.8 May 26 10:04:30 3784.102 99.8 May 26 10:09:07 3784.103 99.8 May 26 10:31:59 3787.001 24.3 May 26 16:06:13 3787.00299 .8 May 26 16:40:27 3787.101 24.3 May 26 15:24:04 3787.102 24.3 May 26 16:03:39 3793.001 24.3 May 27 00:07:34 3793.002 99.8 May 27 00:39:50 3793.003 99.8 May 27 00:43:56 3793.004 99.8 May 27 01:03:37 3793.005 99.8 May 27 03:09:04 3793.006 99.8 May 27 03:15:11 3793.007 99.8 May 27 05:23:31 3793.008 99.8 May 27 05:32:29 3793.009 99.8 May 27 07:32:08 3793.010 99.8 May 27 07:39:39 3793.101 24.3 May 26 23:57:13 3793.10299 .8 May $27 \quad 00: 48: 45$ 3793.103 99.8 May 27 00:51:14 3793.104 99.8 May 27 03:11:35 3793.105 99.8 May 27 03:23:16 3793.106 99.8 May 27 05:26:52 3793.107 99.8 May 27 05:40:42 3793.108 99.8 May 27 07:36:13 3793.109 99.8 May 27 07:50:49 3796.00124 .3 May 27 15:41:17 3796.002 99.8 May 27 16:29:27 3796.003 99.8 May 27 18:51:29 3796.101 24.3 May 27 14:21:09 3796.102 24.3 May 27 15:32:40 3796.103 99.8 May 27 16:15:45 3796.104 99.8 May 27 19:19:45 $\begin{array}{ll}0.25167 & 0.02628\end{array}$

$\begin{array}{ll}0.28158 & 0.02629\end{array}$

$\begin{array}{lll}0.29627 & 0.02462\end{array}$

$\begin{array}{lll}0.21633 & 0.02432\end{array}$

$\begin{array}{ll}0.25876 & 0.02415\end{array}$

$\begin{array}{lll}0.25651 & 0.02439\end{array}$

$\begin{array}{ll}0.24071 & 0.02440\end{array}$

$\begin{array}{lll}0.22963 & 0.02473\end{array}$

$\begin{array}{ll}0.21124 & 0.02465\end{array}$

$\begin{array}{ll}0.24154 & 0.02488\end{array}$

$\begin{array}{lll}0.19056 & 0.02482\end{array}$

$\begin{array}{ll}0.22911 & 0.02482\end{array}$

$\begin{array}{lll}0.22784 & 0.02489\end{array}$

$\begin{array}{lll}0.20311 & 0.02464\end{array}$

$\begin{array}{lll}0.22225 & 0.02490\end{array}$

$\begin{array}{ll}0.26019 & 0.02490\end{array}$

$\begin{array}{ll}0.23326 & 0.02475\end{array}$

$\begin{array}{lll}0.15813 & 0.02474\end{array}$

$\begin{array}{ll}0.39722 & 0.03716\end{array}$

$\begin{array}{ll}0.26126 & 0.03777\end{array}$

$\begin{array}{lll}0.29573 & 0.03732\end{array}$

$\begin{array}{ll}0.30844 & 0.03674\end{array}$

$\begin{array}{ll}0.20964 & 0.03711\end{array}$

$\begin{array}{ll}0.25738 & 0.02428\end{array}$

$\begin{array}{lll}0.20359 & 0.02427\end{array}$

$\begin{array}{lll}-0.06244 & 0.02430\end{array}$

$\begin{array}{lll}0.36262 & 0.03734\end{array}$

$\begin{array}{ll}0.25171 & 0.03718\end{array}$

$\begin{array}{ll}0.33500 & 0.02458\end{array}$

$\begin{array}{ll}0.35602 & 0.02455\end{array}$

$\begin{array}{ll}0.34507 & 0.03669\end{array}$

$\begin{array}{lll}0.29602 & 0.03685\end{array}$

$\begin{array}{ll}0.23034 & 0.03719\end{array}$

$\begin{array}{ll}0.31687 & 0.03678\end{array}$

$\begin{array}{lll}0.25005 & 0.02613\end{array}$

$\begin{array}{ll}0.31590 & 0.02630\end{array}$

$\begin{array}{ll}0.29782 & 0.02616\end{array}$

$\begin{array}{ll}0.24685 & 0.02641\end{array}$

$\begin{array}{ll}0.29172 & 0.02617\end{array}$

$\begin{array}{ll}0.25888 & 0.02633\end{array}$

$\begin{array}{ll}0.33018 & 0.03424\end{array}$

$\begin{array}{ll}0.30489 & 0.03550\end{array}$

$\begin{array}{ll}0.30142 & 0.03541\end{array}$

$\begin{array}{ll}0.27017 & 0.02522\end{array}$

$\begin{array}{ll}0.22861 & 0.02514\end{array}$

$\begin{array}{ll}0.22071 & 0.02522\end{array}$

$\begin{array}{ll}0.20381 & 0.02531\end{array}$

$\begin{array}{ll}0.25691 & 0.02524\end{array}$

$\begin{array}{lll}0.25691 & 0.02524\end{array}$

$\begin{array}{lll}0.30001 & 0.02613\end{array}$

$\begin{array}{ll}0.25584 & 0.02600\end{array}$

$\begin{array}{ll}0.25061 & 0.02646\end{array}$

$\begin{array}{lll}0.40073 & 0.05458\end{array}$

$\begin{array}{ll}0.29091 & 0.02451\end{array}$

$0.24579 \quad 0.02516$

$\begin{array}{lll}0.17259 & 0.02517\end{array}$ 
3797.001 24.3 May 27 20:58:15 3797.002 24.3 May 27 21:38:42 3797.003 99.8 May 27 22:20:48 3797.101 24.3 May 27 20:52:34 3797.102 24.3 May 27 21:16:33 3797.103 99.8 May 27 22:30:03 3797.104 99.8 May 27 22:38:34 3798.00124 .3 May 27 23:37:10 3798.101 24.3 May 27 23:14:30 3799.001 24.3 May 28 00:47:26 3799.00299 .8 May $28 \quad 01: 21: 15$ 3799.003 99.8 May 28 03:19:05 3799.00499 .8 May 28 05:31:52 3799.005 99.8 May 28 07:27:56 3799.009 99.8 May 28 09:57:39 3799.101 24.3 May 28 00:34:05 3799.10299 .8 May $28 \quad 01: 12: 37$ 3799.103 99.8 May 28 03:27:06 3799.104 99.8 May 28 05:20:55 3799.107 99.8 May 28 10:00:28 3801.00124 .3 May 28 13:05:43 3801.002 24.3 May 28 13:34:49 3801.003 99.8 May 28 14:14:17 3801.004 99.8 May 28 16:08:55 3801.10124 .3 May 28 12:58:31 3801.102 24.3 May 28 13:30:10 3801.10399 .8 May 28 14:11:47 3801.104 99.8 May 28 16:16:51 3803.00124 .3 May 28 22:31:04 3803.002 24.3 May 28 22:57:35 3803.003 99.8 May 28 23:17:34 3803.004 99.8 May 28 23:34:00 3803.005 99.8 May 28 23:48:52 3803.006 99.8 May $2901: 37: 48$ 3803.007 99.8 May 29 03:38:23 3803.008 99.8 May 29 05:53:28 3803.009 99.8 May $2907: 35: 48$ 3803.010 99.8 May 29 09:46:51 3803.10124 .3 May 28 22:27:55 3803.102 24.3 May 28 22:50:20 3803.103 99.8 May 28 23:25:41 3803.104 99.8 May 29 01:53:55 3803.105 99.8 May 29 03:48:24 3803.106 99.8 May 29 05:38:41 3803.107 99.8 May 29 07:45:28 3803.11299 .8 May 29 10:58:45 3809.001 24.3 May 29 20:38:35 3809.002 24.3 May 29 21:11:07 3809.003 99.8 May 29 21:28:58 3809.101 24.3 May 29 20:07:11 3809.102 24.3 May 29 20:33:20 3809.103 24.3 May 29 21:04:28 3809.10499 .8 May 29 21:27:19 3810.001 24.3 May 30 00:34:34 3810.002 99.8 May $30 \quad 01: 39: 16$ 3810.00399 .8 May 30 06:23:57
$\begin{array}{ll}0.38870 & 0.02629\end{array}$
$\begin{array}{ll}0.35194 & 0.02636\end{array}$
$\begin{array}{ll}0.24180 & 0.02637\end{array}$
$\begin{array}{ll}0.37761 & 0.02142\end{array}$
$\begin{array}{ll}0.34148 & 0.02214\end{array}$
$\begin{array}{ll}0.20974 & 0.02538\end{array}$
$\begin{array}{ll}0.21012 & 0.02537\end{array}$
$\begin{array}{ll}0.39435 & 0.02696\end{array}$
$\begin{array}{ll}0.35898 & 0.02459\end{array}$
$\begin{array}{ll}0.41027 & 0.03703\end{array}$
$\begin{array}{ll}0.25486 & 0.02622\end{array}$
$\begin{array}{ll}0.20971 & 0.02648\end{array}$
$\begin{array}{ll}0.28786 & 0.02658\end{array}$
$\begin{array}{ll}0.24704 & 0.02625\end{array}$
$\begin{array}{ll}0.01978 & 0.02659\end{array}$
$\begin{array}{ll}0.37344 & 0.03489\end{array}$
$\begin{array}{ll}0.27987 & 0.02521\end{array}$
$\begin{array}{ll}0.27554 & 0.02522\end{array}$
$\begin{array}{ll}0.25642 & 0.02530\end{array}$
$\begin{array}{ll}0.03165 & 0.02549\end{array}$
$\begin{array}{ll}0.39621 & 0.02640\end{array}$
$\begin{array}{ll}0.34855 & 0.02643\end{array}$
$\begin{array}{ll}0.27028 & 0.02638\end{array}$
$\begin{array}{ll}0.29479 & 0.02641\end{array}$
$\begin{array}{ll}0.37862 & 0.02866\end{array}$
$\begin{array}{ll}0.34989 & 0.02298\end{array}$
$\begin{array}{ll}0.28212 & 0.02539\end{array}$
$\begin{array}{ll}0.25143 & 0.02558\end{array}$
$\begin{array}{ll}0.27198 & 0.02551\end{array}$
$\begin{array}{ll}0.32013 & 0.02604\end{array}$
0.176820 .02562
$\begin{array}{ll}0.20470 & 0.02581\end{array}$
$0.21024 \quad 0.02596$
$\begin{array}{ll}0.26030 & 0.02607\end{array}$
$0.21274 \quad 0.02635$
$\begin{array}{ll}0.19314 & 0.02628\end{array}$
$\begin{array}{ll}0.19775 & 0.02645\end{array}$
$\begin{array}{ll}0.17026 & 0.02648\end{array}$
$\begin{array}{ll}0.32016 & 0.02471\end{array}$
$\begin{array}{ll}0.33720 & 0.02273\end{array}$
$\begin{array}{ll}0.27332 & 0.02636\end{array}$
$\begin{array}{ll}0.23474 & 0.02649\end{array}$
$\begin{array}{ll}0.23105 & 0.02659\end{array}$
$\begin{array}{ll}0.20028 & 0.02650\end{array}$
0.251420 .02668
$\begin{array}{lll}0.24287 & 0.04664\end{array}$
$\begin{array}{ll}0.28532 & 0.02647\end{array}$
$\begin{array}{ll}0.29378 & 0.02590\end{array}$
$\begin{array}{ll}0.20815 & 0.02623\end{array}$
$\begin{array}{ll}0.24136 & 0.02558\end{array}$
$\begin{array}{ll}0.27323 & 0.01840\end{array}$
$\begin{array}{ll}0.27664 & 0.02583\end{array}$
$\begin{array}{ll}0.15123 & 0.02613\end{array}$
$\begin{array}{ll}0.25829 & 0.02580\end{array}$
$\begin{array}{ll}0.17282 & 0.02611\end{array}$
$0.17846 \quad 0.02640$

3810.004 99.8 May 30 07:49:01

3810.101 24.3 May 30 00:29:13

3810.102 99.8 May $30 \quad 01: 44: 50$

3810.103 99.8 May 30 06:21:49

3810.104 99.8 May $30 \quad 07: 47: 16$

3811.002 99.8 May 30 13:15:02

$\begin{array}{ll}0.22010 & 0.02032\end{array}$

$\begin{array}{ll}0.11567 & 0.02593\end{array}$

0.134790 .02628

$0.09474 \quad 0.02647$

$0.00321 \quad 0.03041$ $\begin{array}{ll}0.20640 & 0.02633\end{array}$

\section{REFERENCES}

1. C. E. Allgower et al., Phys. Rev. D, 65, 092008 (2002).

2. J. Tojo et al., Phys. Rev. Lett., 89, 052302 (2002).

3. T. L. Trueman, hep-ph/0305085 (2003).

4. W.R. Lozowski and J.D. Hudson, NIM in Physics Research, A334, 173 (1993).

5. I.G. Alekseev et al., AIP Conf. Proc., 675, 812 (2003).

6. H. Paul (2003), original data of the stopping power for the light ions was taken from the web site: www.exphys.uni-linz.ac.at/stopping.

7. D. Kawall, Muon g-2 note No322 (1998). 